\title{
COMMISSIONING AND OPERATION OF THE FNAL FRONT END INJECTION LINE AND ION SOURCES
}

Patrick R. Karns

Submitted to the faculty of the University Graduate School in partial fulfillment of the requirements for the degree Master of Science in the Department of Physics, Indiana University, 
Accepted by the Graduate Faculty, Indiana University, in partial fulfillment of the requirements for the degree of Master of Science.

Master's Thesis Committee

Dr. Shyh-Yuan Lee, Chairperson

Dr. Cheng-Yang Tan

Dr. Mike Snow

Dr. Rex Tayloe 


\section{Acknowledgments}

I would like to acknowledge Cheng-Yang Tan who was my incredibly patient primary advisor for this thesis work at FNAL. I would also like to acknowledge Shyh-Yuan Lee, my thesis defense committee chair and the members of the committee, Dr. Mike Snow, and Dr. Rex Tayloe. I would also like to thank William Barletta, Irina Novitski, and especially Susan Winchester for managing the USPAS program and making the logistics of this program easy to navigate. I would like to thank Bill Pellico and Fernanda G. Garcia for allowing me to participate in this program. I would also like to thank Dan Bollinger for all his help and guidance and turning a thesis project into a vocation in Sourcery. I would also like to thank Andrew Feld, Ken Koch, Kent Triplett, Jeff Larson, Kevin Duel, Mike Kucera, Dean Arveson, Bob Florian, Vic Scarpine, Jim Lackey, Chuck Schmidt, Ray Tomlin, Brian Schupbach, Janet Schmidt, and Benjamin Koubek without whom this project would have been infinitely harder to complete.

This thesis work was completed at Fermi National Accelerator Laboratory, operated by Fermi Research Alliance, LLC under Contract No. De-AC02-07CH11359 with the United States Department of Energy 
Patrick R. Karns

\section{COMMISSIONING AND OPERATION OF THE FNAL FRONT END INJECTION LINE AND ION}

\section{SOURCES}

This thesis documents the efforts made in commissioning and operating the RFQ Injection Line (RIL) as a replacement for the Cockcroft Walton front end. The Low Energy Beam Transport (LEBT) was assembled and tested with multiwire position and emittance monitor measurements. The Radio Frequency Quadrupole (RFQ) commissioning was completed with the same measurements as well as output beam energy measurements that showed it initially accelerated beam only to $700 \mathrm{keV}$, which was $50 \mathrm{keV}$ lower than the design energy. Working with the manufacturer solutions were found and instituted to continue testing. The Medium Energy Beam Transport (MEBT) was then connected as the RIL was installed as the new front end of Linac. Testing gave way to operation when the new front end was used as the source of all High Energy Physics (HEP) beam for Fermi National Accelerator Laboratory (FNAL). The magnetron ion source that provides the $\mathrm{H}^{-}$beam for the front end required several changes and eventual upgrades to operate well; such as new source operating points for vacuum pressure and cesium admixture, and new materials for critical source components. Further research was conducted on the cathode geometry and nitrogen doping of the hydrogen gas as well as using solid state switches for the extractor system high voltage. 


\section{Table of Contents}

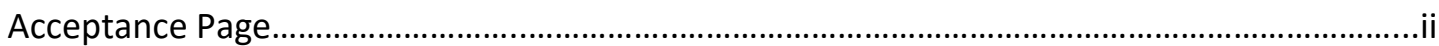

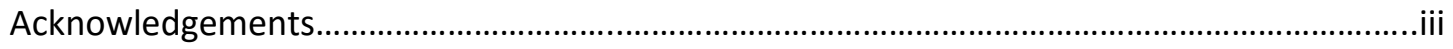

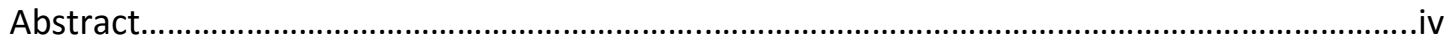

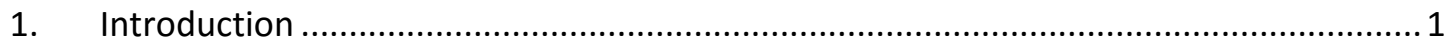

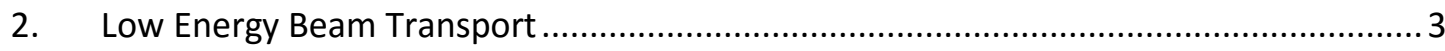

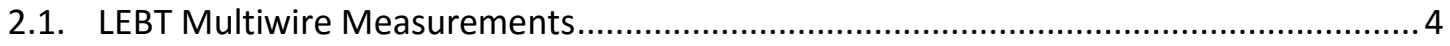

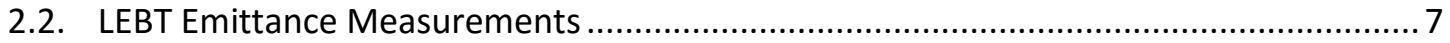

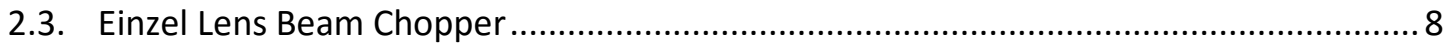

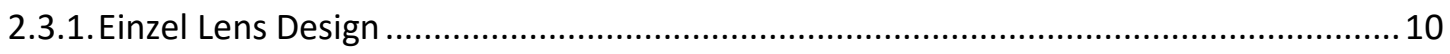

2.3.2. Einzel Lens Operation with Solid State Switches ............................................. 11

2.3.3. Rise and Fall Time Measurements ........................................................... 13

2.4. Neutralization and Toroid Measurements.................................................... 15

3. Radio Frequency Quadrupole .................................................................. 17

3.1. RFQ Output Energy Measurements ...................................................... 19

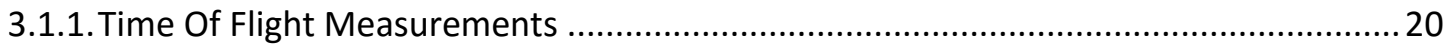

3.1.2.Spectrometer Magnet Measurements ......................................................... 21

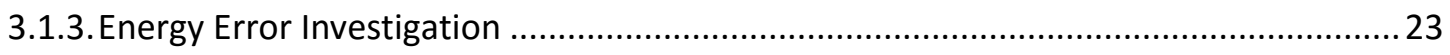

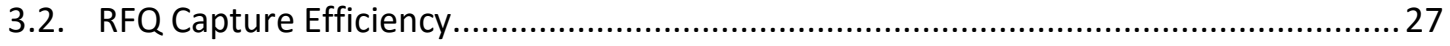

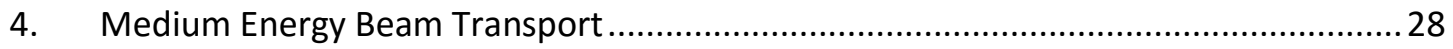




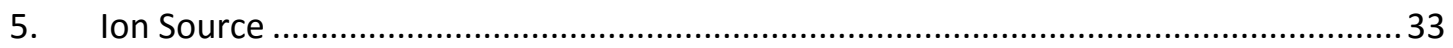

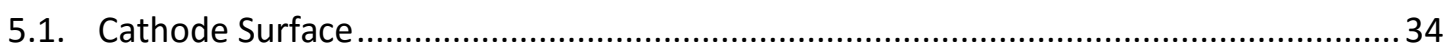

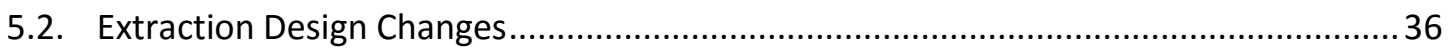

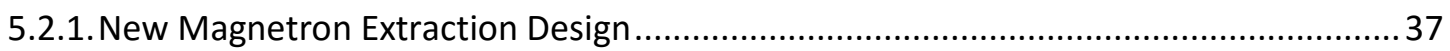

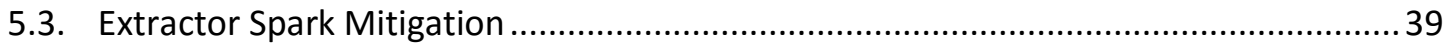

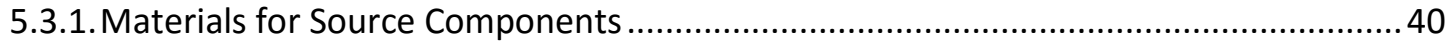

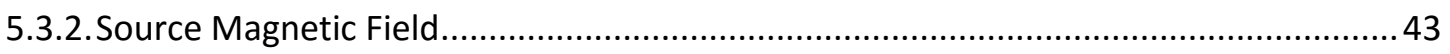

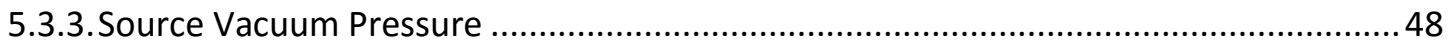

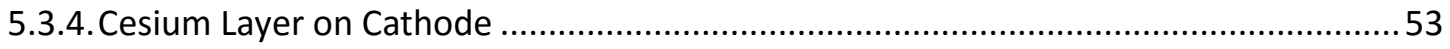

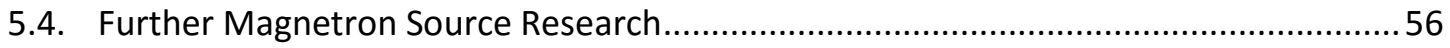

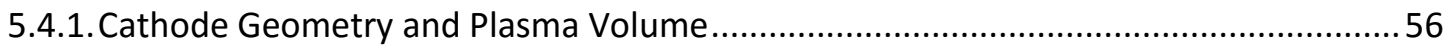

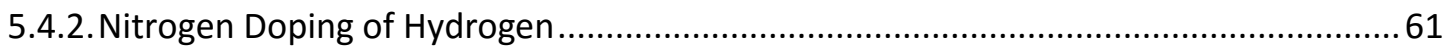

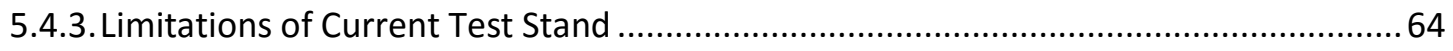

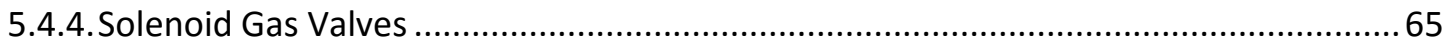

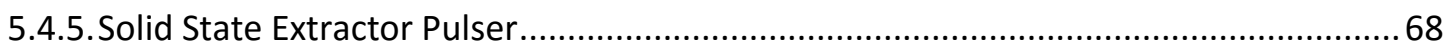

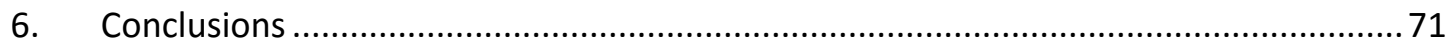

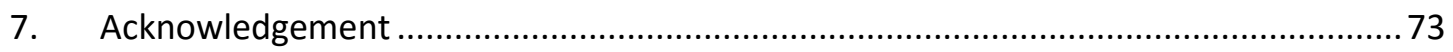

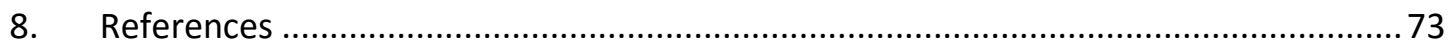

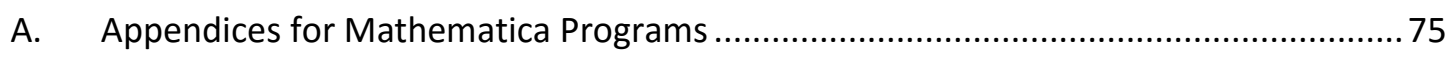

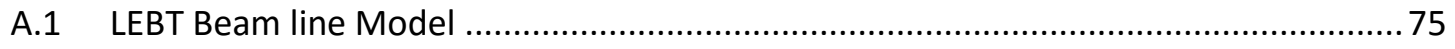




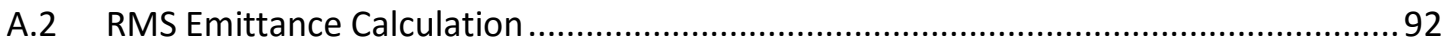

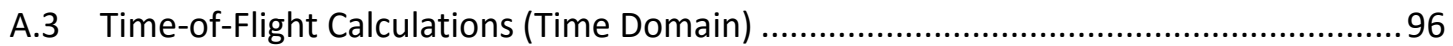

A.4 Time-of-Flight Calculations (Frequency Domain) .......................................................99

$\mathrm{CV}$ 


\section{Introduction}

The Fermi National Accelerator Laboratory (FNAL) High Energy Physics (HEP) program had relied on Cockcroft-Walton accelerators to provide $\mathrm{H}$ - ion beams to the Linac accelerator from 1978 until 2012. Two such accelerators were operated during this time in a redundant fashion where one would provide the $750 \mathrm{keV} \mathrm{H}$ - beam to the Linac while the other one was kept in stand-by in case of failure. As the systems became older, components became harder to repair or replace as these parts were no longer manufactured and the personnel responsible for the system's maintenance and operation had retired. This loss of expertise coupled with more frequent and lengthy accelerator downtime caused by the Cockcroft-Walton accelerators required a replacement to be designed, tested, and installed.
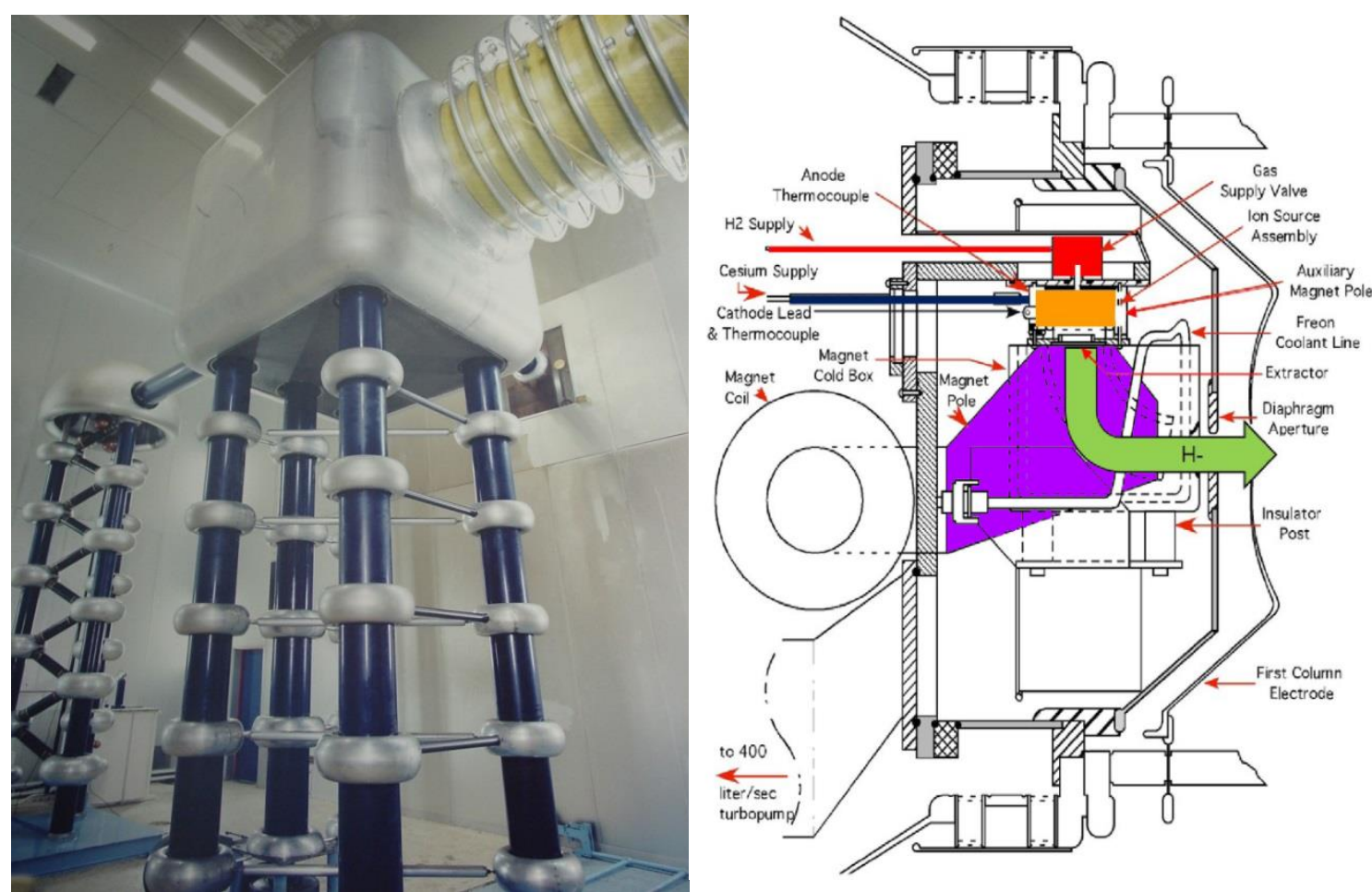

Figure 1: The FNAL Cockcroft-Walton accelerator (left). This was one of two systems operated for over 40 years. A close up drawing of the ion source assembly (right) shows the main parts of the system to create and accelerate $\mathrm{H}^{-}$ ions. 
The Cockcroft-Walton systems shown in Figure 1 included a magnetron style $\mathrm{H}$ - source, slitextracted [1] at roughly $17 \mathrm{keV}$ downwards into a 90 degree bend magnet that injected into the acceleration column. The source body, magnet, associated controls and electronics were housed inside a dome at $-750 \mathrm{kV}$ potential relative to the room walls. The accelerating column connected the dome and source inside to the ground potential wall and accelerated the $\mathrm{H}$ beam to $750 \mathrm{keV}$ in gradual steps to limit electrical breakdown and sparking in the column. Upon exiting the column the beam was then chopped, having portions selected for insertion into the Linac accelerator, or dumped into a carbon disk. The chopper used two charged plates that alternated charge states to either select beam or discard it into the carbon disk. Between this and the Linac was a 90 degree bend magnet used to select the beam of the operational source and other beam line magnets used to match the beam optics to the Linac. All of this was replaced in the upgrade in the summer of 2012 [2].

The new system can be broken into four major parts; The Source Cube, Low Energy Beam Transport (LEBT), the Radio Frequency Quadrupole (RFQ), and the Medium Energy Beam Transport (MEBT). All together, the system is called the RFQ Injection Line or RIL for short. The line from the ion source up to and including the RFQ was built and tested in the ion source lab to ensure the system could provide an $\mathrm{H}^{-}$ion beam suitable to replace the Cockcroft-Walton sources.

In order to show that the installation of the RIL caused no harm to the FNAL accelerator complex, the new system had to be able to at a minimum replicate the beam that had been provided by the Cockcroft Walton accelerators. These had provided $46 \mathrm{~mA}$ or more to the FNAL Linac with transverse emittances typically $(1.2-2) \pi \mathrm{mm}$ mrad in each plane. This allowed the Linac to provide $35 \mathrm{~mA}$ to the Booster accelerator which would then be able to deliver greater than $4.5 \times 10^{12}$ protons per pulse to the Main Injector or to $8 \mathrm{GeV}$ experiments. As long as the 
Booster accelerator was still able to provide this intensity to the downstream machines or users, variances on these requirements would be allowable.

Another thing that needed to be improved was the uptime of the machine. The Cockcroft Walton generators being 40 years old was a major factor in the downtime of the systems requiring maintenance or repairs. Increasing this uptime to over $95 \%$ was a crucial requirement for this upgrade.

\section{Low Energy Beam Transport}

The Low Energy Beam Transport (LEBT) is the short beam line that connects the source cube to the RFQ. The purpose of this beam line is to take the highly divergent beam that exits the source cube and focus it transversely into a small radius, and transport it to the entrance of the RFQ. The final function of the LEBT is to select which portions of the source beam pulse is to be allowed into the RFQ and accelerated to $750 \mathrm{keV}$. This is typically done with a beam chopper magnet, but in this case an Einzel Lens is used as a 'chopper' to stop and/or reflect the $\mathrm{H}^{-}$beam before it reaches the RFQ. The beam line is shown in Figure 2 along with the RFQ.

The main focusing elements in the beam line consist of two large solenoids. The first is situated near the exit of the source cube. This solenoid brings the divergent $\mathrm{H}^{-}$ion beam from the source into a paraxial beam that travels down the LEBT. The second solenoid is used to focus this $35 \mathrm{keV}$ beam into the $5 \mathrm{~mm}$ RFQ rod aperture.

There are three pairs of dipole trims to aid in directing the beam through the LEBT. Each pair has one horizontal and one vertical trim housed in the same assembly. The first trim is mounted on the exit of the source cube to direct beam through the first solenoid. The second trim pair lies downstream of the first solenoid to point the beam through the vacuum break 
section that houses the toroid used to measure beam intensity in the LEBT. A third trim pair sits after the vacuum equipment section just upstream of the second solenoid to aim the beam through the solenoid and RFQ.

The last element in the LEBT is the Einzel lens chopper. An Einzel lens is used here as a mirror, reflecting the $\mathrm{H}^{-}$beam instead of focusing the beam as it enters the RFQ. This Einzel lens chopper takes up very little space between the LEBT and the RFQ. This is the first time an Einzel lens has been implemented in this manner in an accelerator (See Section 2.3).

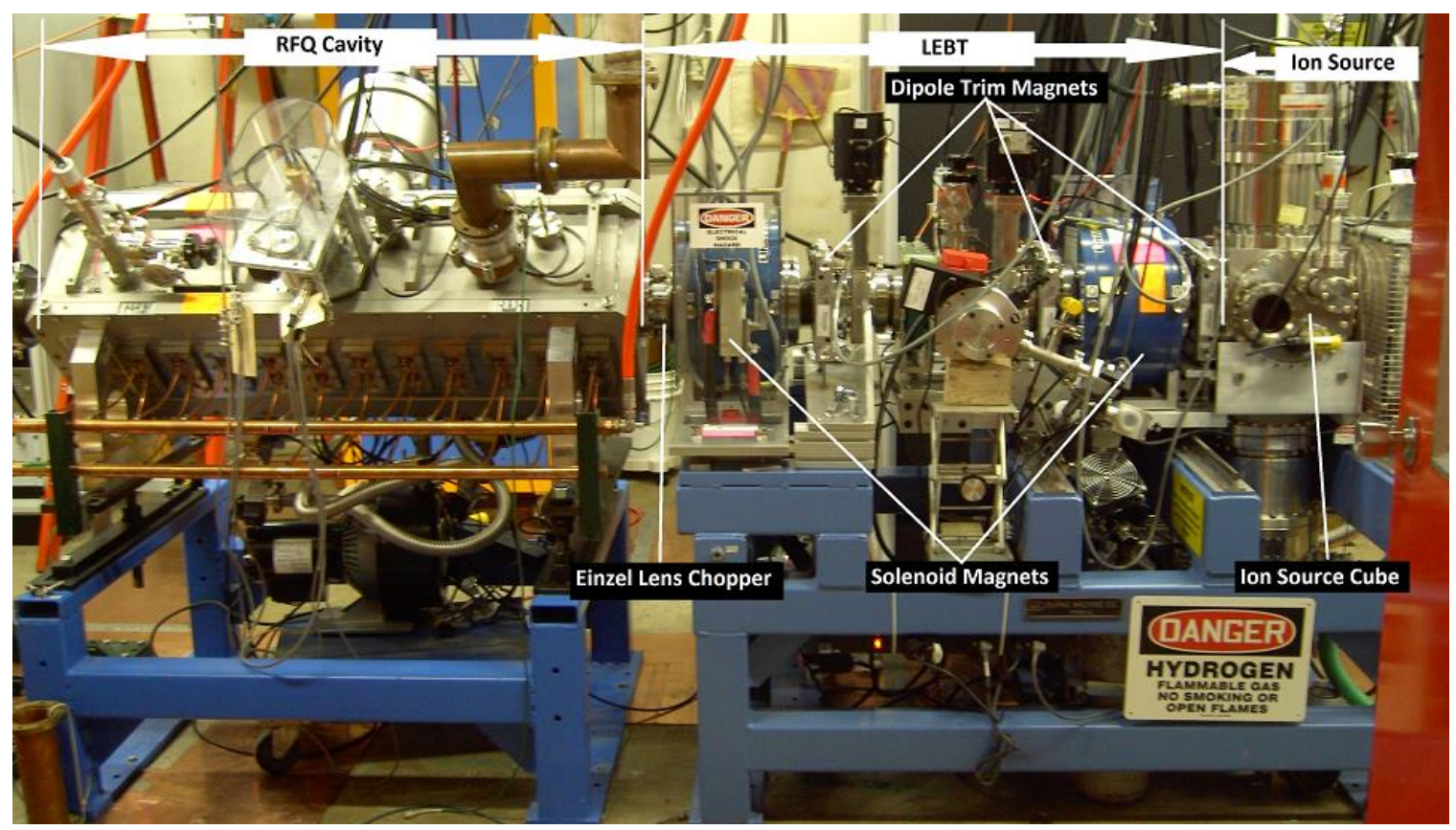

Figure 2: The RFQ Injection Line assembled in the test stand. The three main sections from right to left are the lon Source, LEBT, and RFQ.

\subsection{LEBT Multiwire Measurements}

A model of the beam line was created in Mathematica using the matrix formalism for beam line optics. This Mathematica code is detailed in Appendix A.1. Using this model shown in Figure 3a, we were able to determine not only the approximate starting values for the solenoid 
current but also values of the trim dipoles to direct the beam to a position of our choosing on a set of multiwires.

Testing began with quantifying the effects of the trim dipoles on the beam through the LEBT assembled seen in Figure 3b. A multiwire beam scanner was installed at the end of the LEBT at the longitudinal position of the RFQ aperture and was used to measure beam profiles in both the horizontal and vertical transverse planes.

a)

After alignment on 13 Mar 2012, multiwires end of LEBT

b)
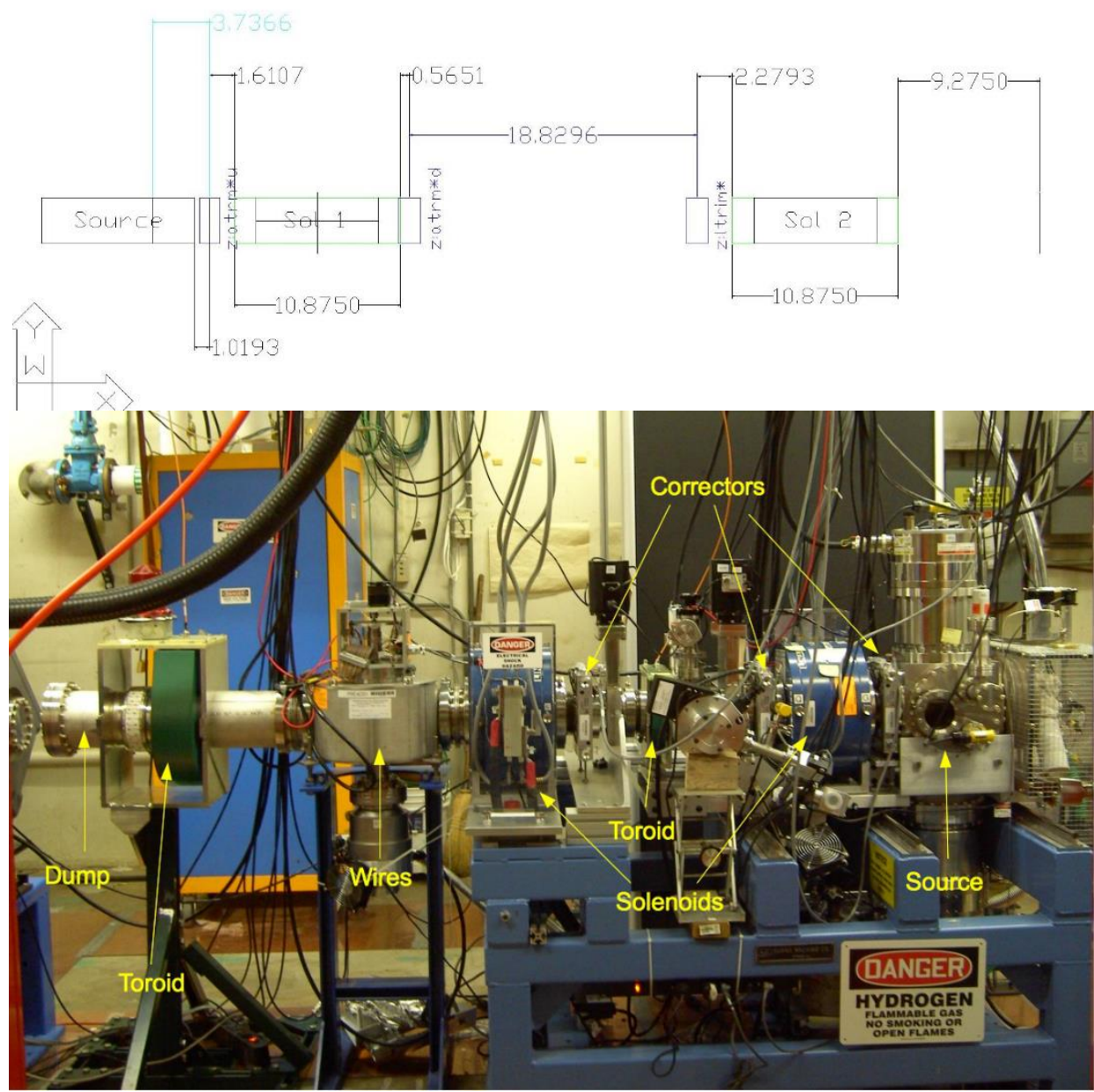

Figure 3: (a) The beamline model showing the distances between each element as measured during the test setup. Beam direction in the model diagram is left to right. (b) The LEBT beam line as tested with the multiwire and toroid installed. Beam direction in the beam line picture is right to left. Note that the wire can's longitudinal position is the same as the RFQ in Figure 2. 
For a given value of solenoid current, each trim was scanned over its entire range, from -4 A to $+4 \mathrm{~A}$ in $1 \mathrm{~A}$ increments. Figure 4 shows an overlay of one scan in one plane for one trim. Each trim required scans in both planes regardless of its designed plane of interaction as the solenoids rotated the beam through the line and coupled the transverse motion. These scans could be then combined to calculate the beam position change as a function of the current in each trim as shown in Figure 5. This data could then be inserted into beam line model for the LEBT to help plan trim currents to aim the beam into the RFQ aperture.

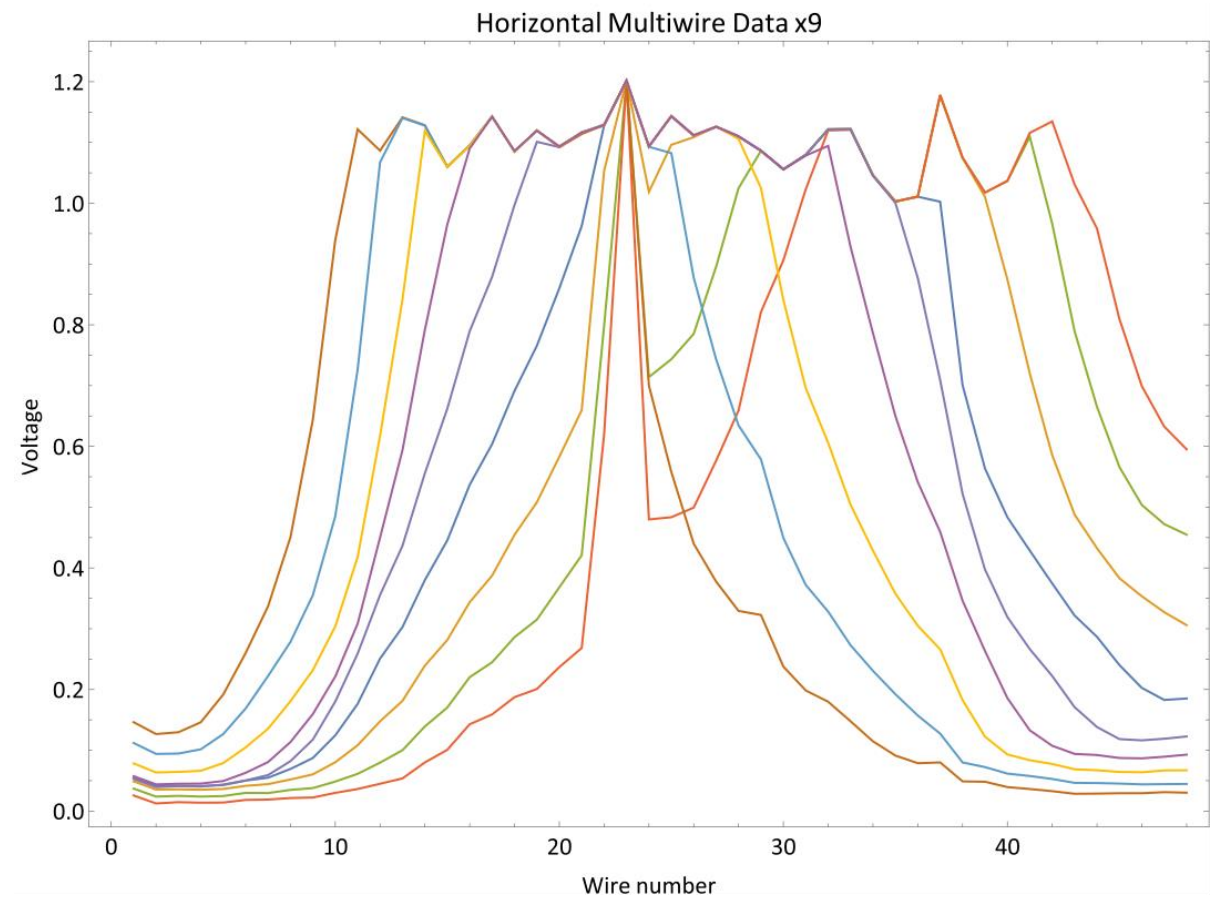

Figure 4: An overlay of 9 multiwire measurements for one LEBT trim. Each trace is the data for one of the trim settings from -4 to $4 \mathrm{~A}$. The multiple traces show the beam centroid moving across the multiwires. 


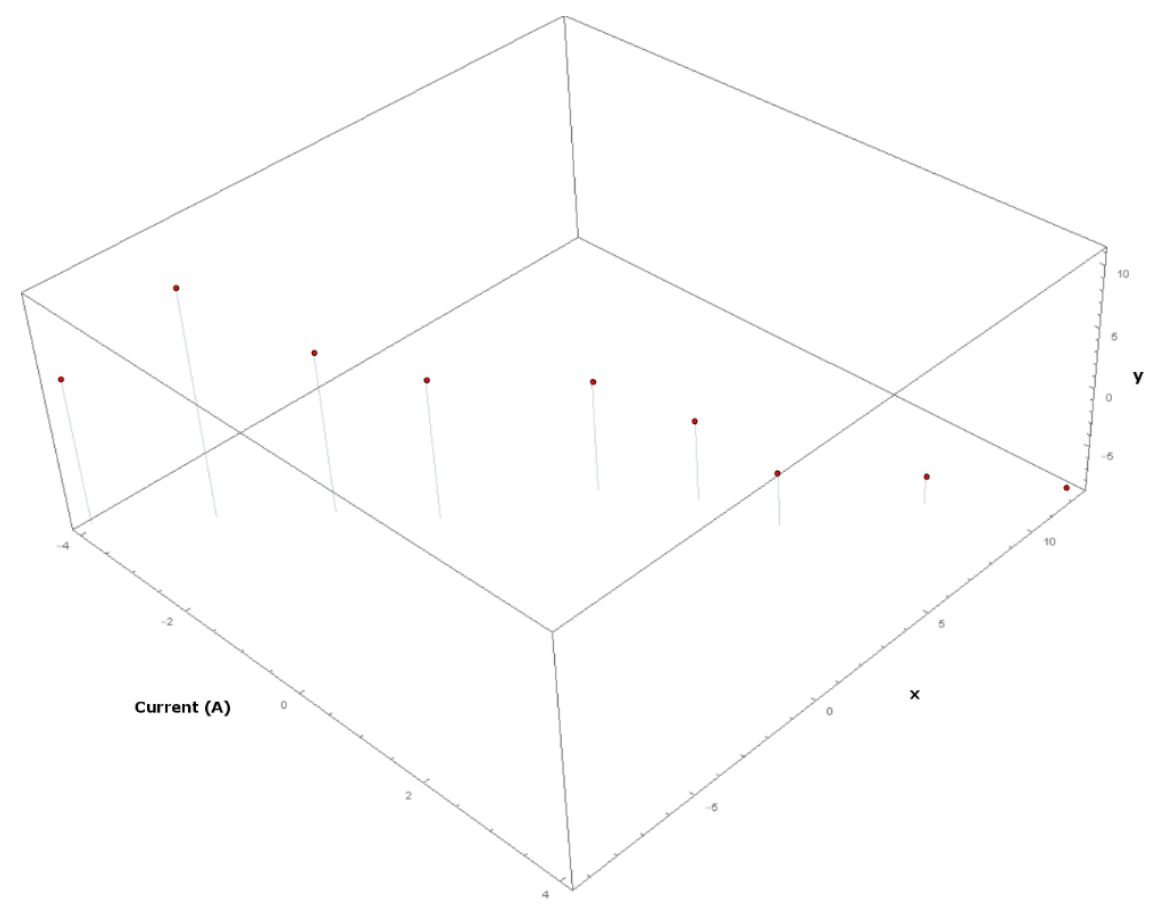

Figure 5: The multiwire data from both the horizontal and vertical planes are combined to show the beam motion across the aperture as a result of the current change in one dipole trim.

\subsection{LEBT Emittance Measurements}

Emittance scans were also used to characterize the beam being injected into the RFQ.

These scanners were installed at the same position as the multiwires where the RFQ entrance would be. The scanners used for these measurements were the slit and wire type. In this detector a small slit was set $2 \mathrm{in}$. before a grid of 20 wires spaced $0.01 \mathrm{in}$. apart. This spacing gives an angular spread of 5 mrad per wire of the detector. The slit and wires are moved across the beam in $10 \mathrm{~mm}$ steps. At each of these steps, beam passes through the slit and the interrupted charge is seen as a current that is proportional to it on the wires. The RMS emittances were calculated by the data taken from the probes that were analyzed offline using Mathematica code shown in Appendix A.2 initially.

An unexpected revelation from the emittance probe measurements was that the beam exiting the LEBT was not round. The vertical emittance was consistently larger than the 
horizontal as shown in Figure 6. A review of the beam line model did not explain this difference in transverse beam sizes.

This discrepancy was traced back to the permanent magnets used in the ion source. The magnets are strong enough to spread the beam vertically as it exits the source. These magnets will be covered later in Section 5.3.2. This was verified by rotating the ion source by $90^{\circ}$ and measuring the emittance again to find the horizontal emittance was now consistently larger than the vertical emittance.
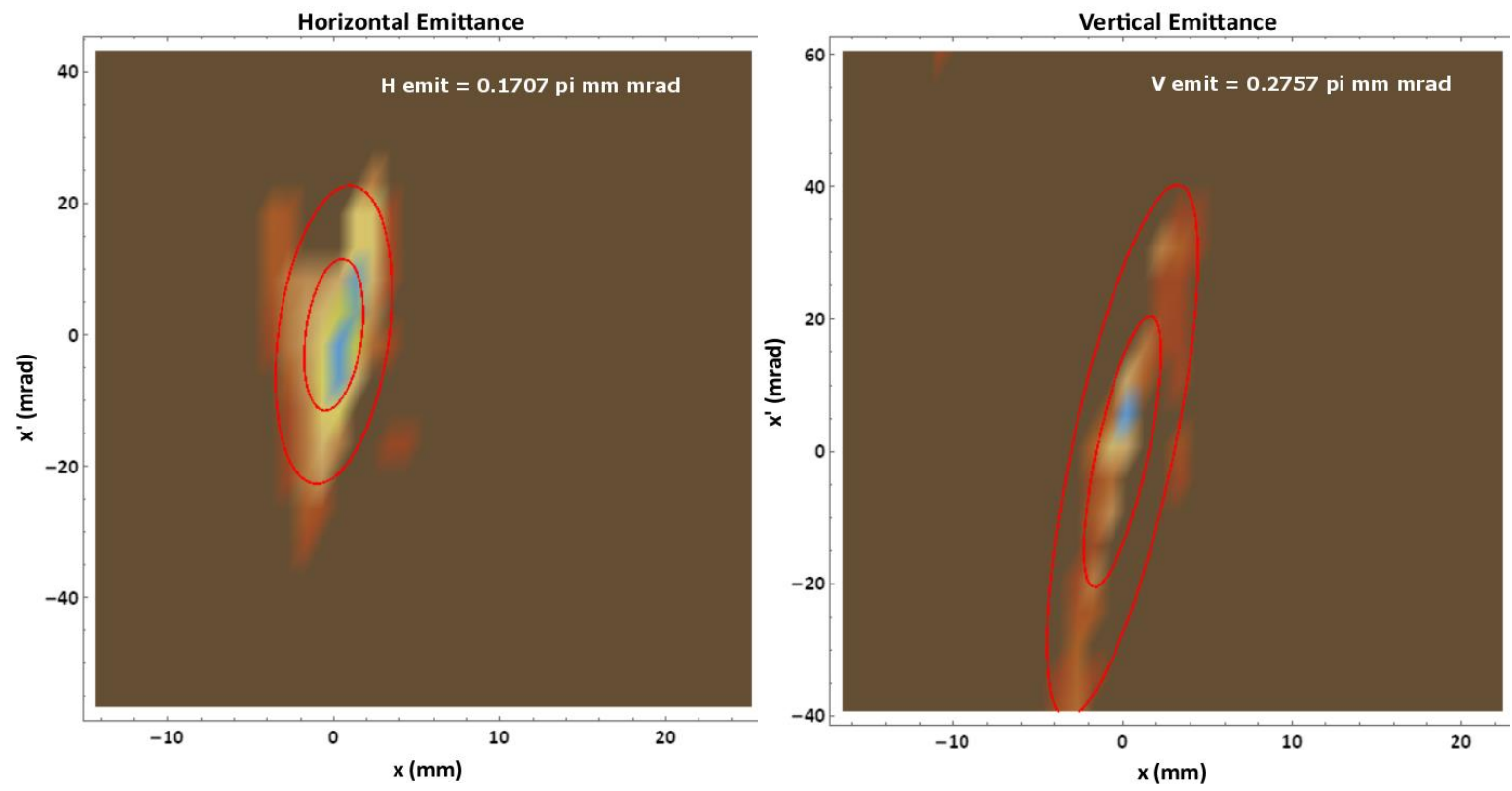

Figure 6: The horizontal (left) and vertical (right) emittance phase space plots for $35 \mathrm{keV}$ beam. The vertical emittance is consistently higher than the horizontal emittance. The fit ellipses are calculated as shown in Appendix A.2. The expected emittance numbers were $0.3 \pi \mathrm{mm}$ mrad in both planes at the entrance to the RFQ as supplied by the manufacturer [2].

\subsection{Einzel Lens Beam Chopper}

A novel solution was required for the beam chopper with the tight constraints on the length of the LEBT and placement of the RFQ. The old $750 \mathrm{keV}$ line used a pair of electrostatic charged plates to divert the unwanted beam to a carbon disk. Even at $35 \mathrm{keV}$ this would have required 
longer plates than space was available. There was also the issue of de-neutralization that the chopper plates would cause that would defocus the beam before entering the RFQ.

The Einzel Lens was presented as a possible solution that could be mounted near to the RFQ and be able to stop the $35 \mathrm{keV}$ beam [3]. In this position, the de-neutralization effects on the $\mathrm{H}^{-}$ beam would be minimal because the lens is very short.

The Einzel Lens is not used as a focusing element in this beam line. An Einzel Lens used for focusing would place the three electrodes at different potentials to create a strong focusing effect on the beam. The outer two electrodes of the Einzel Lens used as a chopper are grounded and the center electrode is pulsed to $-38.5 \mathrm{keV}$. This Einzel Lens acts as a mirror, or reflector that causes the $35 \mathrm{keV} \mathrm{H}^{-}$beam to stop or turn around, thus preventing beam from entering the RFQ.

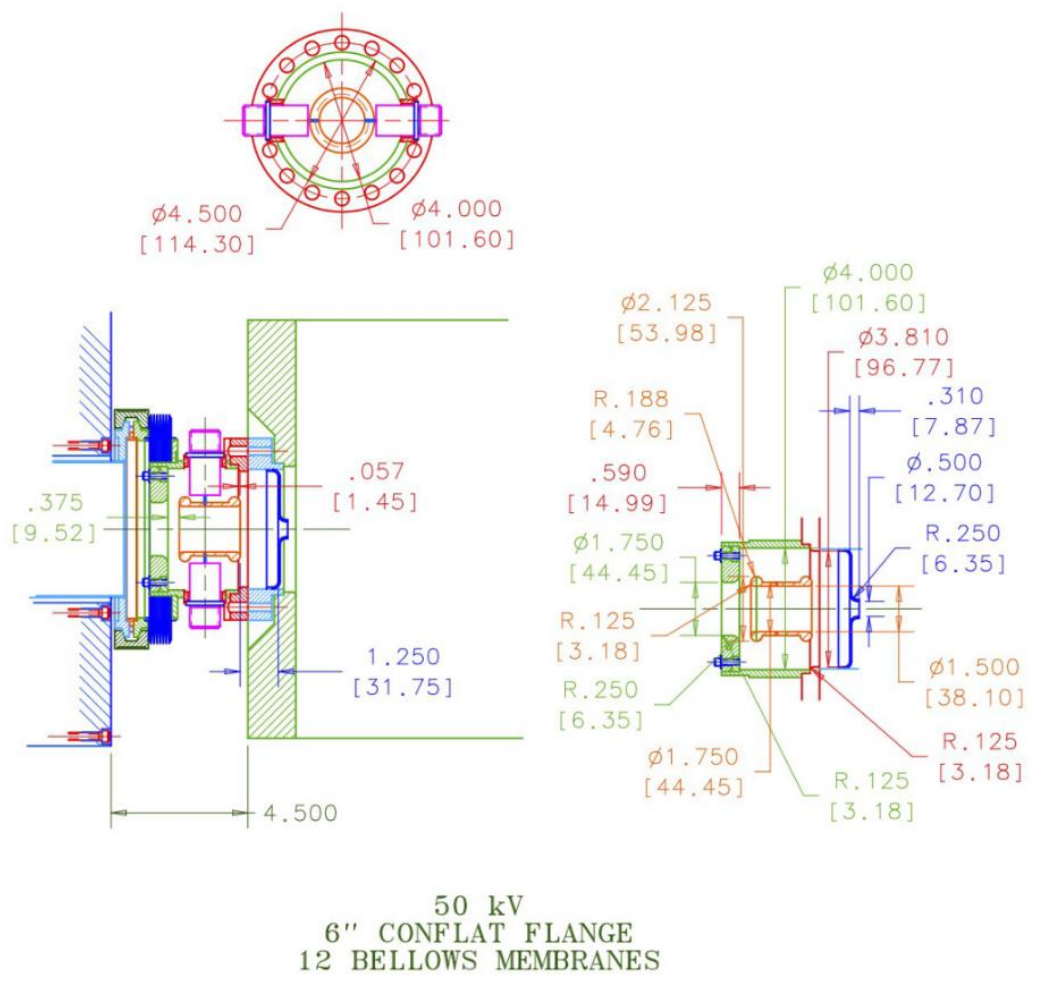

Figure 7: Mechanical drawing of the first Einzel Lens design. 


\subsubsection{Einzel Lens Design}

The Einzel Lens design must be radially large enough so as to not create an aperture restriction for beam between the solenoid and the RFQ, but must also be able to hold $\geq 35 \mathrm{kV}$ potential at its center. The chosen design shown in Figure 7 is a lens that is 2 in. long and 1.75 in. in diameter and mounted directly on the upstream flange of the RFQ with a potential of 38.5 $\mathrm{kV}$ to be applied to the lens. The extra $3.5 \mathrm{kV}$ above the beam energy is needed to ensure the potential at the center of the lens would be $\geq 35 \mathrm{kV}$ to stop or reflect the $35 \mathrm{keV}$ beam.

After one year of operation, the lens began to have problems maintaining $38.5 \mathrm{kV}$ and began sparking under vacuum. Upon removing the lens, tracks in newly deposited thin metal plating had become visible on the ceramic isolators that held the lens in place inside the enclosure as shown in Figure 8. A spare lens was put into operation while a re-design of the lens was started to solve the problems found in the original design.

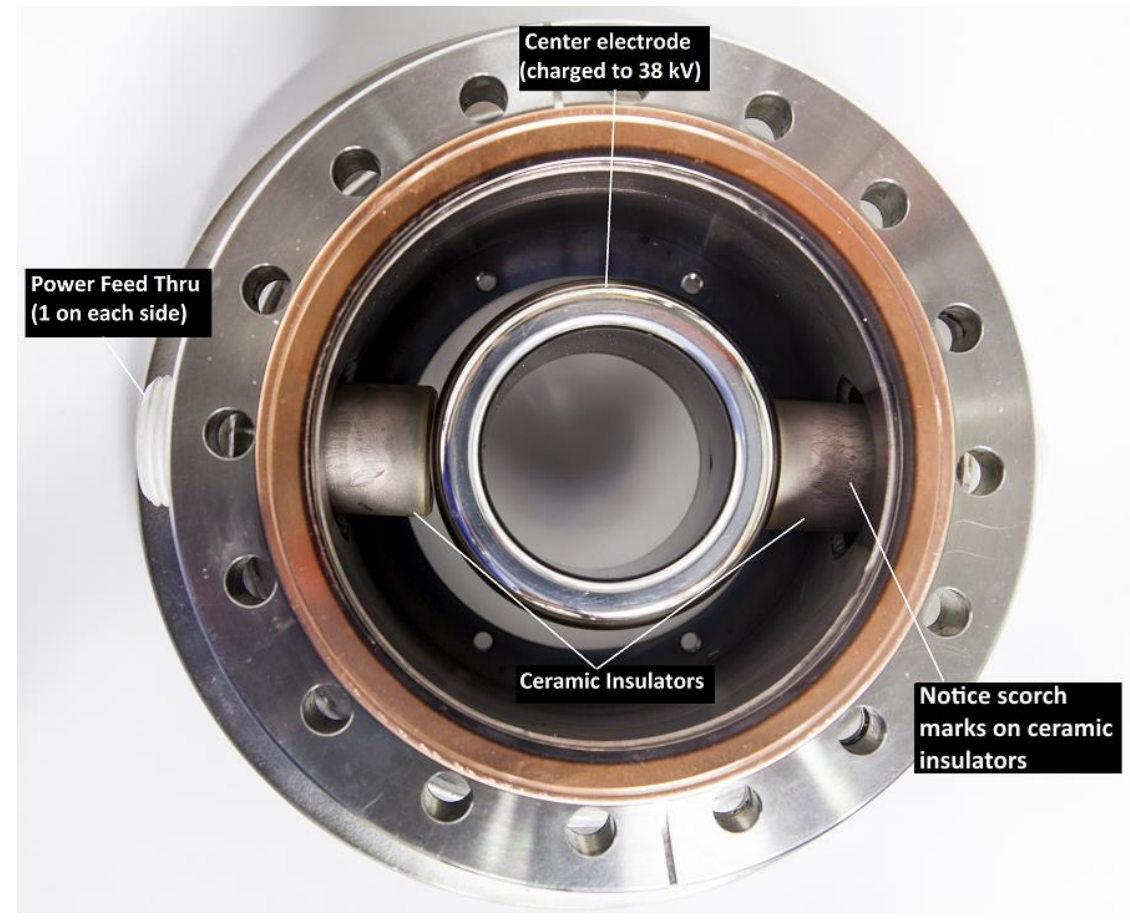

Figure 8: The Einzel Lens chopper. This is the initial design after one year of use. Note the scorch marks and metal plating on the ceramic insulators. 
During the 2014 accelerator maintenance period, the re-designed lens was installed and tested. It failed almost immediately with sparking internal to the vacuum chamber. This design had created a triple point where the metal for the lens, the ceramic insulator, and the vacuum met where the electric field gradient was high enough to allow sparking. A cleaned and reconditioned model of the original design was put in place while the lens design started another revision. Until such time as a suitable re-design is found, the current plan is to swap operational models of the first design each year during the accelerator maintenance period, cleaning and recertifying the removed lens for use as a spare, or to be installed in the following year.

\subsubsection{Einzel Lens Operation with Solid State Switches}

The FNAL Linac is capable of accelerating $\mathrm{H}^{-}$beam pulses at a $15 \mathrm{~Hz}$ repetition rate. Each pulse varies in length from $2.2 \mu$ s to $62 \mu$ s during standard operations. In order for the Einzel Lens to function as a beam chopper, the rise and fall times of the charging system must be short enough to be able to pulse on and off and recover between each $15 \mathrm{~Hz}$ pulse.

To achieve the desired repetition rate and variable pulse lengths, solid state IGBT switches were procured from Diversified Technologies Inc. for the charging system These switch assemblies are rated at $50 \mathrm{kV}$ and $50 \mathrm{~A}$ and the associated control and driver electronics were provided with the switches. A custom enclosure was built and it is shown in Figure 9. Two switch assemblies were used so that the lens would be quickly discharged as well as charged. 


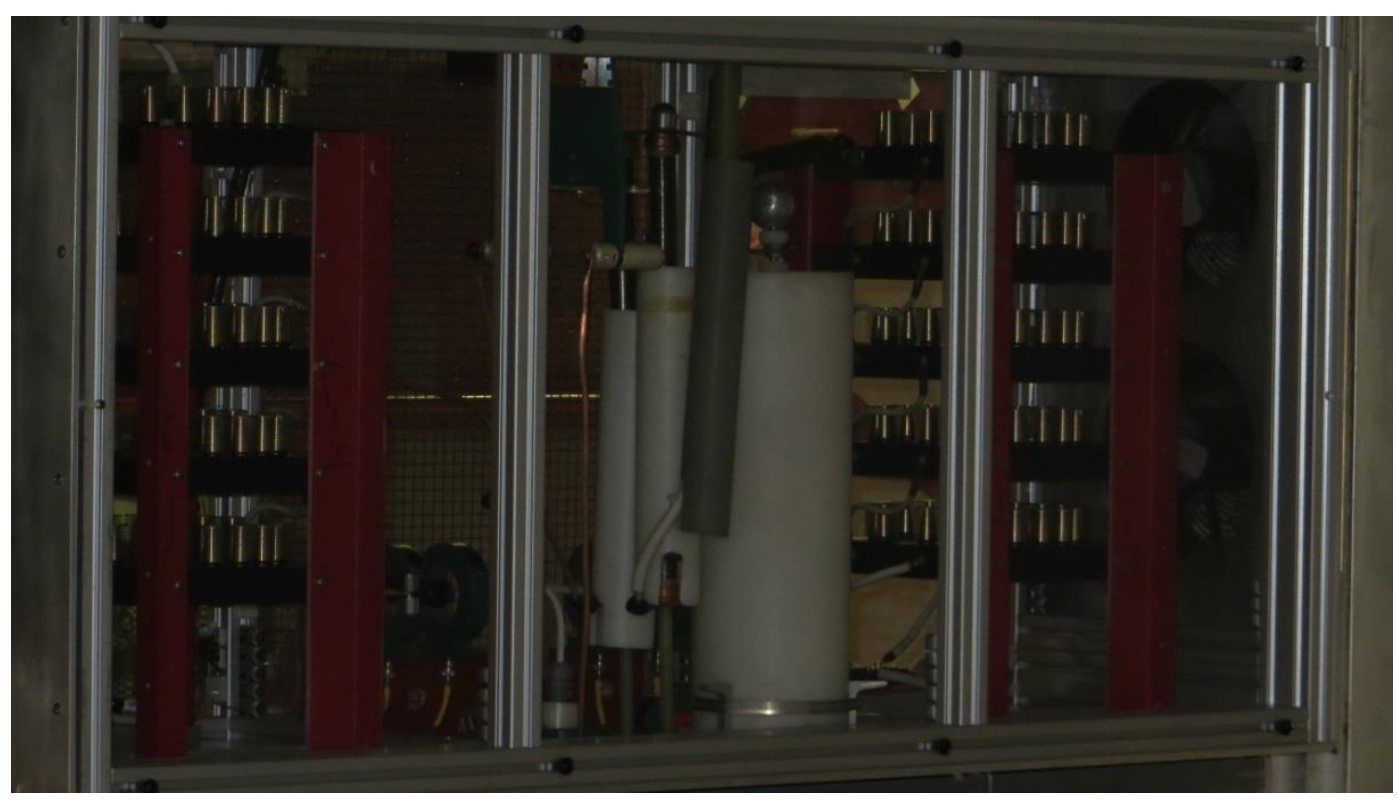

Figure 9: The Einzel Lens solid state switch cabinet. The solid state switch stacks are on either side with the large white capacitor in the center. Current sensing toroids for system protection can also be seen in the center.

A diagram showing the timing and cycling of the switches is shown in Figure 10. Before the ion source even begins to create the $\mathrm{H}^{-}$beam, the first switch (SW1) is turned on to prevent beam from entering the RFQ before it is requested. When beam is desired through the RFQ, SW1 is turned off, and the second switch (SW2) is turned on to discharge the lens. Once the desired length of beam pulse has been allowed through the RFQ, SW2 is turned off and SW1 is turned back on, thus preventing beam from passing into the RFQ again. This switch is held on until after the ion source has stopped creating beam and then SW1 is again turned off. The lens voltage is allowed to decay away until another beam pulse is requested during the next $15 \mathrm{~Hz}$ cycle. 


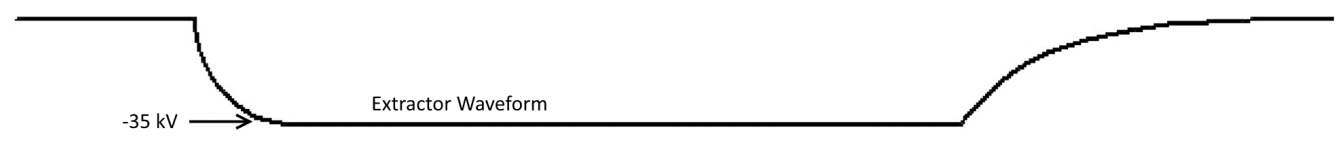

Arc Discharge Current

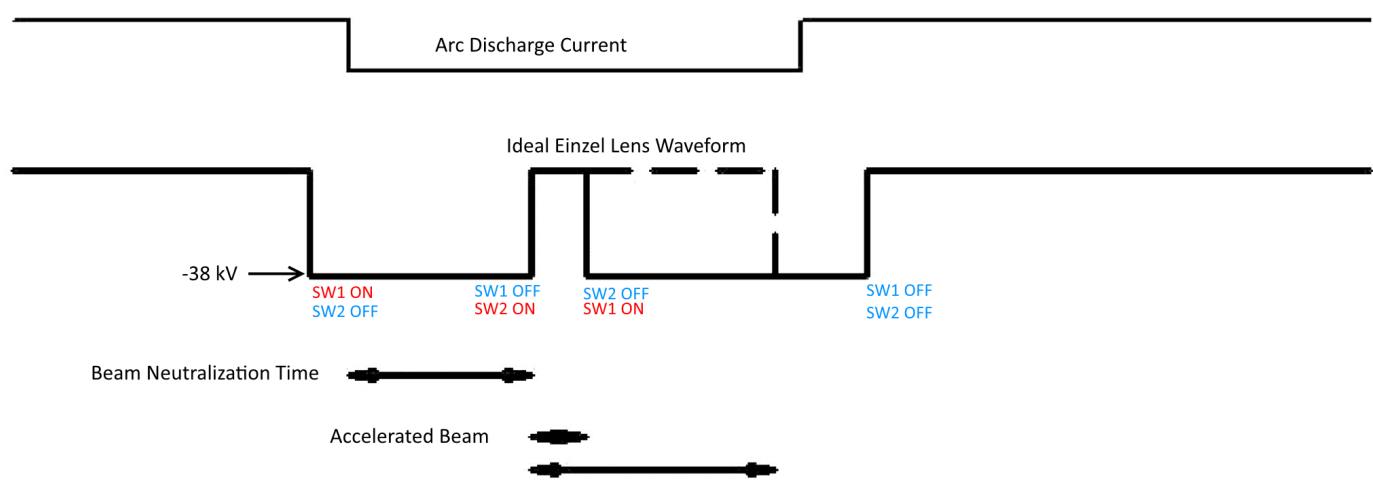

Figure 10: A diagram showing Einzel Lens pulse timing relative to the other source timers. Note the Einzel Lens is turned on before the Arc supply to prevent beam from entering the RFQ until requested.

\subsubsection{Rise and Fall Time Measurements}

Measuring the rise and fall times of the Einzel Lens required a fast Faraday cup $(15-20 \mathrm{GHz}$ bandwidth) to be placed downstream of the lens. A voltage of $38.5 \mathrm{kV}$ was determined to ensure that there was no beam leaking outside of the intended pulse length. This was verified by looking at a toroid placed downstream of the Einzel Lens and verifying no shoulders were apparent on the toroid signal as shown in Figure 11.

Once there were no apparent shoulders as seen on the toroid signal, the Faraday cup was placed into the beam and the signals from it were measured on a $6 \mathrm{GHz}$ bandwidth oscilloscope. The Faraday cup signals shown in Figure 12 were averaged 16x with background subtracted and give a rise time of $138 \mathrm{~ns}$ and a fall time of $81 \mathrm{~ns}$. 


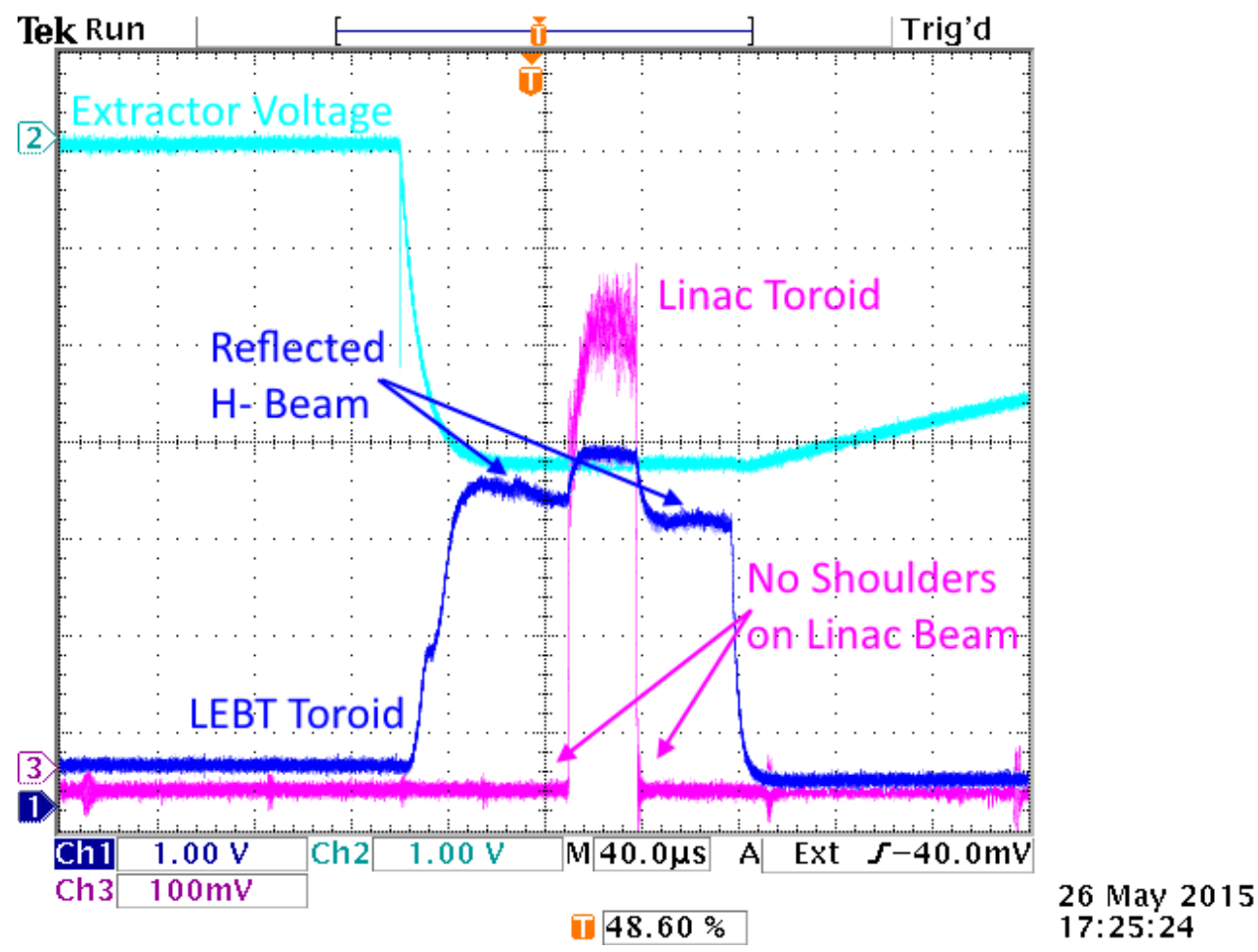

Figure 11: Oscilloscope trace showing extractor voltage with toroid signals before and after the Einzel Lens. The Einzel Lens is set to $38.5 \mathrm{kV}$ and the effect of the reflected beam can be seen on the LEBT toroid.

The larger rise time is due to the stray capacitances that arise during charging of the system and increase the total charging capacitance versus the discharging capacitance. It would be possible to reduce both the rise and fall times by shorting out one stack of the IGBT switches. Each switch stack contributes $150 \Omega$, for a total of $750 \Omega$. This was not pursued because the rise time of 138 ns was within the requirements for the Einzel Lens chopper.

The Einzel Lens creates a peculiar beam signal on the LEBT toroid as a result of it reversing the direction of travel for some of the beam. The lens reflects a portion of the $\mathrm{H}^{-}$beam back through the toroid reducing the apparent beam signal on the toroid. A backwards travelling negative beam ends up being subtracted from the forward going $\mathrm{H}^{-}$beam while the Einzel Lens has voltage on it and can be seen in the toroid signals of Figure 11. 


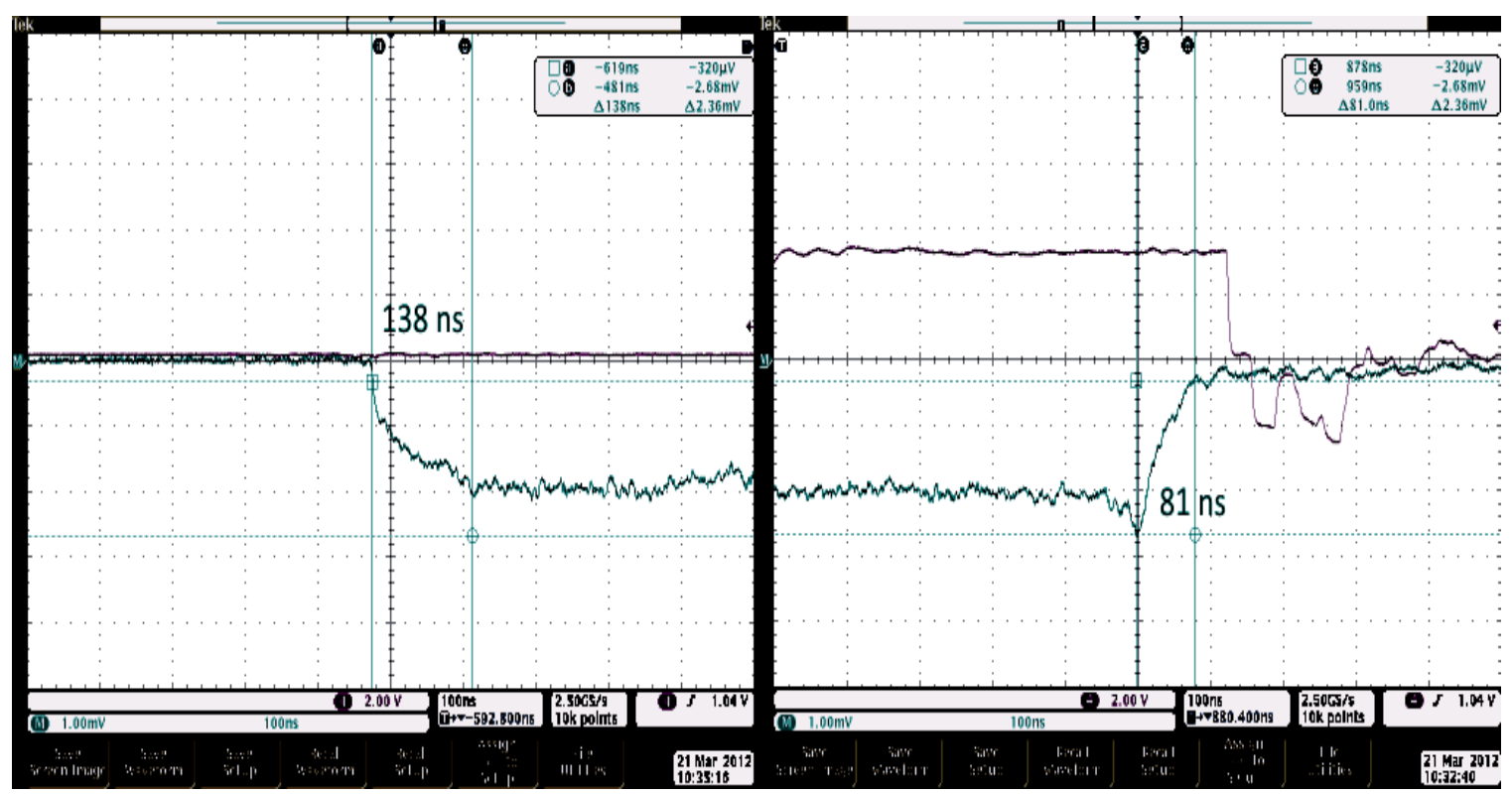

Figure 12: Oscilloscope traces showing the rise (a) and fall (b) times of the pulsed voltage on the lens. The rise time is larger due to stray capacitances that charge up during lens charging.

\subsection{Neutralization and Toroid Measurements}

The $\mathrm{H}^{-}$ion beam that is traveling down the LEBT is an intense, space charge dominated beam. Even with the strong focusing by the solenoids, beam line transfer efficiency suffers from the defocusing effect of the space charge of the $\mathrm{H}^{-}$beam. This beam loss can be mitigated somewhat with self-focusing due to charge neutralization.

Xenon gas was proposed as a suitable gas for neutralization due to its short neutralization time period of roughly $40 \mu \mathrm{s}$ [4]. Initial tests for the neutralization effect were conducted with hydrogen gas due to its presence in the LEBT as a result of the low efficiency of the magnetron source in converting hydrogen gas into $\mathrm{H}^{-}$ions. Enough hydrogen gas leaks from the source cube into the LEBT through the connecting aperture that the Xenon gas method was not pursued. The neutralization time for hydrogen is $60 \mu \mathrm{s}$. 
The Einzel Lens chooses which part of the 220 us beam pulse to allow into the RFQ, which is usually near the middle of the beam pulse. Even with a long beam pulse of $62 \mu$ s the total pulse length needed to allow for neutralization with hydrogen plus the $\mathrm{H}^{-}$beam pulse is only $122 \mu \mathrm{s}$.

While the $\mathrm{H}^{-}$beam transits the LEBT, the $\mathrm{H}^{-}$beam interacts with the residual gas in the beam line. The beam knocks electrons off the hydrogen atoms in the beam pipe and scatters the electrons to the walls of the beam pipe. The remaining positive ions then create a positive potential well that pulls the $\mathrm{H}^{-}$ions toward them, in effect focusing the $\mathrm{H}^{-}$beam.

Proper vacuum pressure for neutralization using remnant hydrogen was determined experimentally by varying the vacuum pumping in the LEBT. The vacuum pumping is provided by two $350 \mathrm{~L} / \mathrm{s}$ turbo pumps. The pump controllers are capable of varying the speed of the turbo pump from $425 \mathrm{~Hz}$ to $825 \mathrm{~Hz}$ which translates to a pressure ranging from $2 \times 10^{-6}$ Torr to $8 \times 10^{-6}$ Torr, with both pumps running. A plot showing the varying pump speeds, and their effect on the vacuum pressure, and toroid signals is shown in Figure 13. 


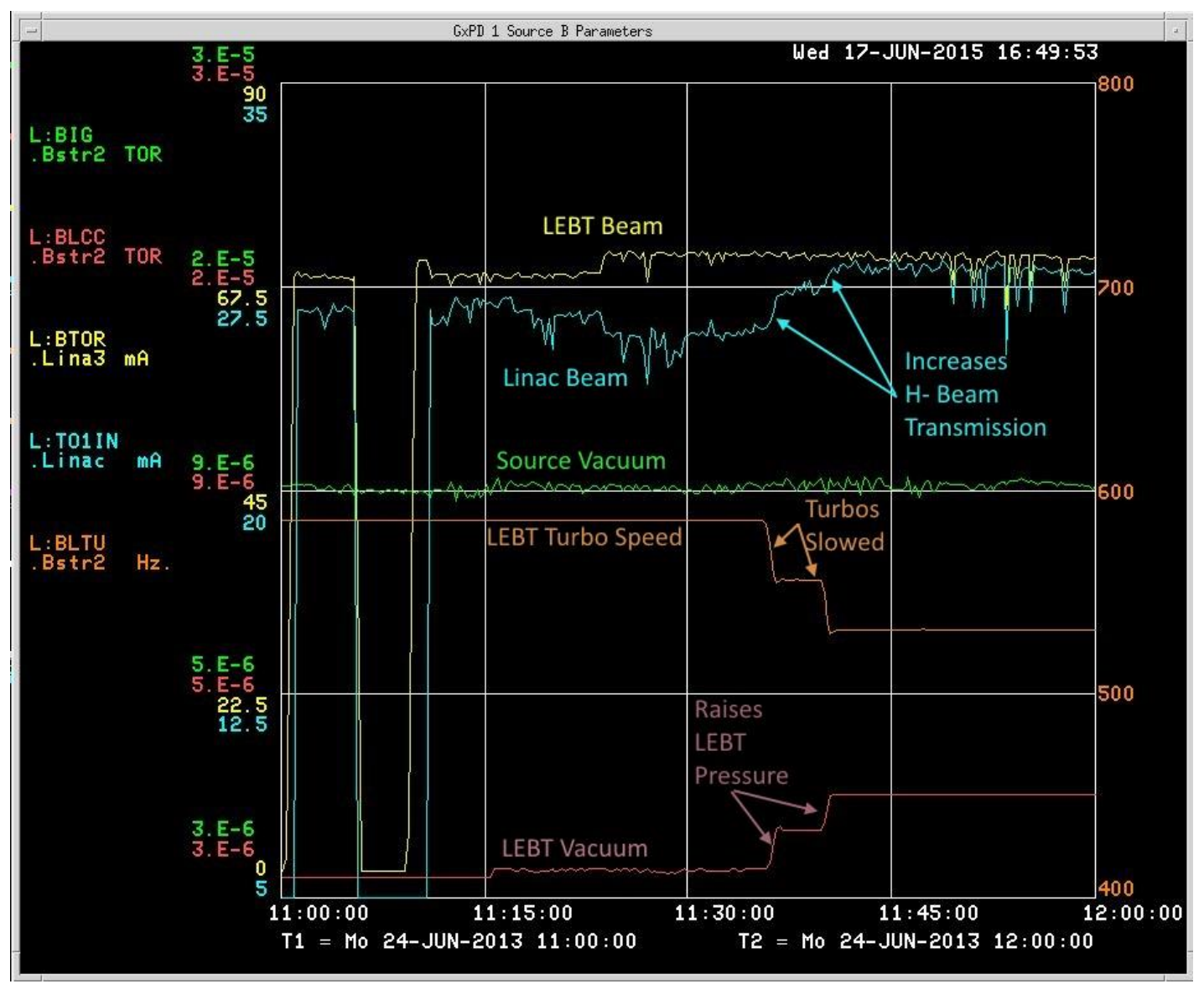

Figure 13: Data plot showing the effects of ion neutralization on beam efficiency in the LEBT. Slowing turbo pumps to raise the pressure increased beam output of the RIL. Linac beam input increased without a similar increase in LEBT beam.

\section{Radio Frequency Quadrupole}

A Radio Frequency Quadrupole (RFQ) is a special kind of RF cavity that not only accelerates the beam, but focuses and bunches it as well. This is achieved via specially shaped electrodes within the cavity placed in close proximity of the beam. The surface of these electrodes closest to the beam is undulated to shape the electric field to accelerate and bunch the beam all at once. These undulations increase in length along the length of the electrodes as the beam energy increases and can be seen in Figure 14. The beam arrives at the exit of the RFQ accelerated to a higher energy and bunched into discrete packets of particles. 
The RFQ that replaced the Cockcroft-Walton Preaccelerators due to its smaller footprint and higher energy efficiency is shown with its vacuum tank opened in Figure 14. A rod style RFQ manufactured by A. Schempp [6] was chosen and was delivered for initial testing in August, 2011. The important parameters are shown in Table 1.

\begin{tabular}{|l|l|l|}
\hline \multicolumn{3}{|c|}{ Table 1 } \\
\hline \multicolumn{1}{|c|}{ Parameter } & \multicolumn{1}{c|}{ Value } & \multicolumn{1}{c|}{ Unit } \\
\hline Input Energy & 35 & $\mathrm{keV}$ \\
\hline Output Energy & 750 & $\mathrm{keV}$ \\
\hline Frequency & 201.25 & $\mathrm{MHz}$ \\
\hline Length & 120 & $\mathrm{~cm}$ \\
\hline Design current & 60 & $\mathrm{~mA}$ \\
\hline $\begin{array}{l}\text { Transmission } \\
\text { efficiency }\end{array}$ & 98 & $\%$ \\
\hline
\end{tabular}

Table 1: Table containing important RFQ design parameters.

Once the RFQ was placed under vacuum and conditioned with low power ( $70 \mathrm{~W} \mathrm{CW}$ ) and with high power ( $100 \mathrm{~kW}$ pulsed) the resonant frequency and the tuning slug range were verified at high power. Once the RFQ was ready, it was connected to the LEBT beam line for beam measurements. Problems were found with its output energy, capture efficiency, and power coupling that required further investigation and troubleshooting to repair. 


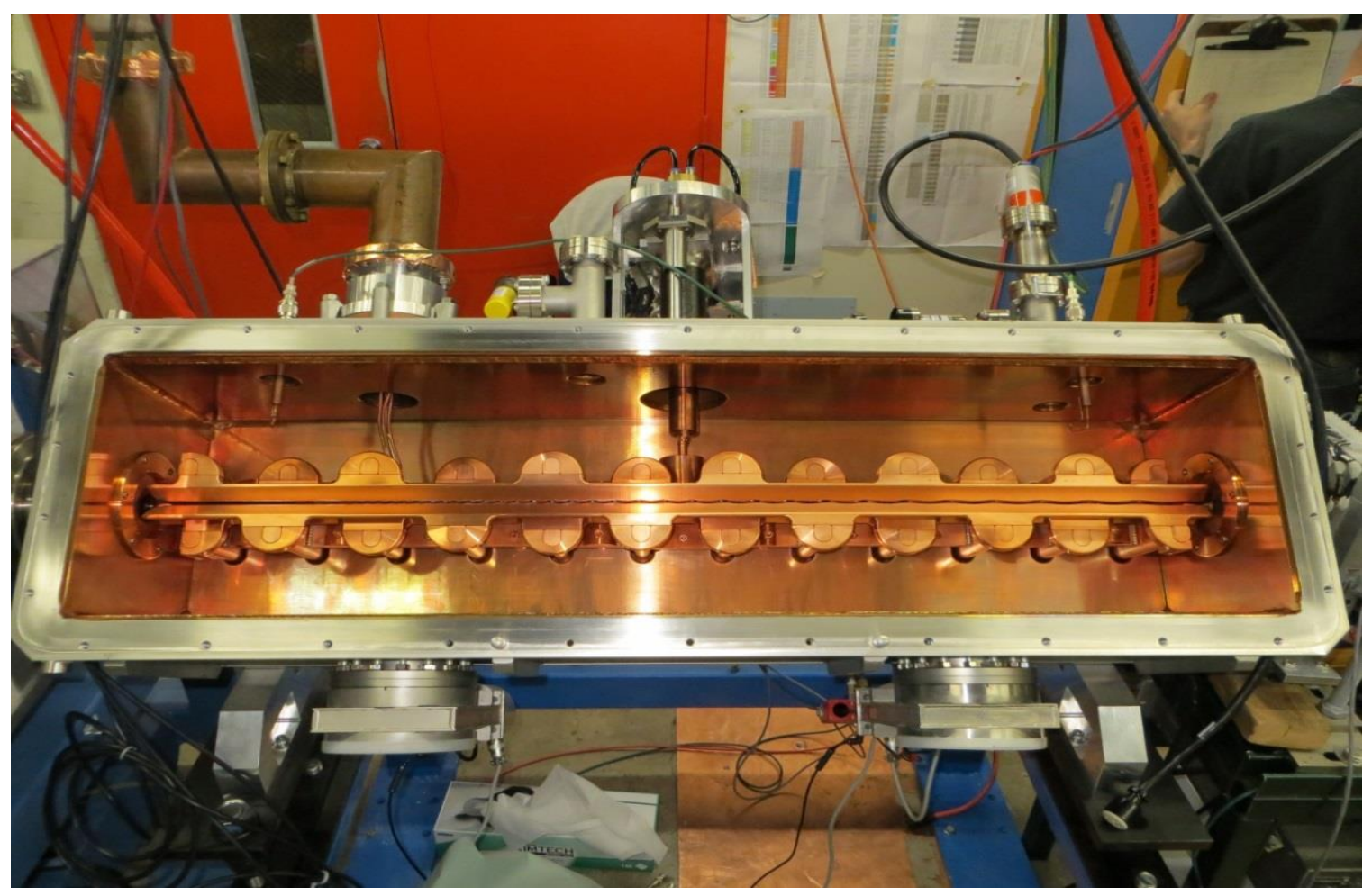

Figure 14: An inside view of the rod type RFQ created by A. Schempp used in the RIL. The inside is copper plated with steel on the outside. The four rod electrodes in the center of the cavity provide the bunching and acceleration of the $\mathrm{H}^{-}$beam as it passes through the cavity. RF input is at top left with the tuning slug at top middle. Two turbo pumps are mounted on the bottom and a third on the lid which is not shown here.

The initial measurements of the RFQ beam output were found to have a far lower intensity than were expected from the specifications. With an input intensity of $\sim 60 \mathrm{~mA}$ the expected intensity downstream of the RFQ was $58 \mathrm{~mA}$. Instead what was seen on the toroid was 46-48 $\mathrm{mA}$, roughly $78 \%$ transmission efficiency. Several causes of this poor efficiency were found, however not all of them were resolvable.

\subsection{RFQ Output Energy Measurements}

The RFQ output energy was measured using two different methods; a time-of-flight (TOF) method using three beam position monitors with a fast sampling oscilloscope, and an energy spectrometer method using a spectrometer magnet and the multiwire assembly used for the LEBT beam line measurements. 


\subsubsection{Time Of Flight Measurements}

The TOF measurement method involves 3 BPM's whose location along the beam line are precisely known, especially with respect to one another. Using this precise distance measurement one can measure the time that each BPM registers the beam pulse and calculate the velocity of the beam and thus the energy of the beam. The setup for this measurement is shown in Figure 15 and the distances between each BPM pair used to calculate the energy of the beam is shown in Table 2 along with the energy calculated from each pair. These energy measurements were made with $180 \mathrm{~kW}$ power in the RFQ.

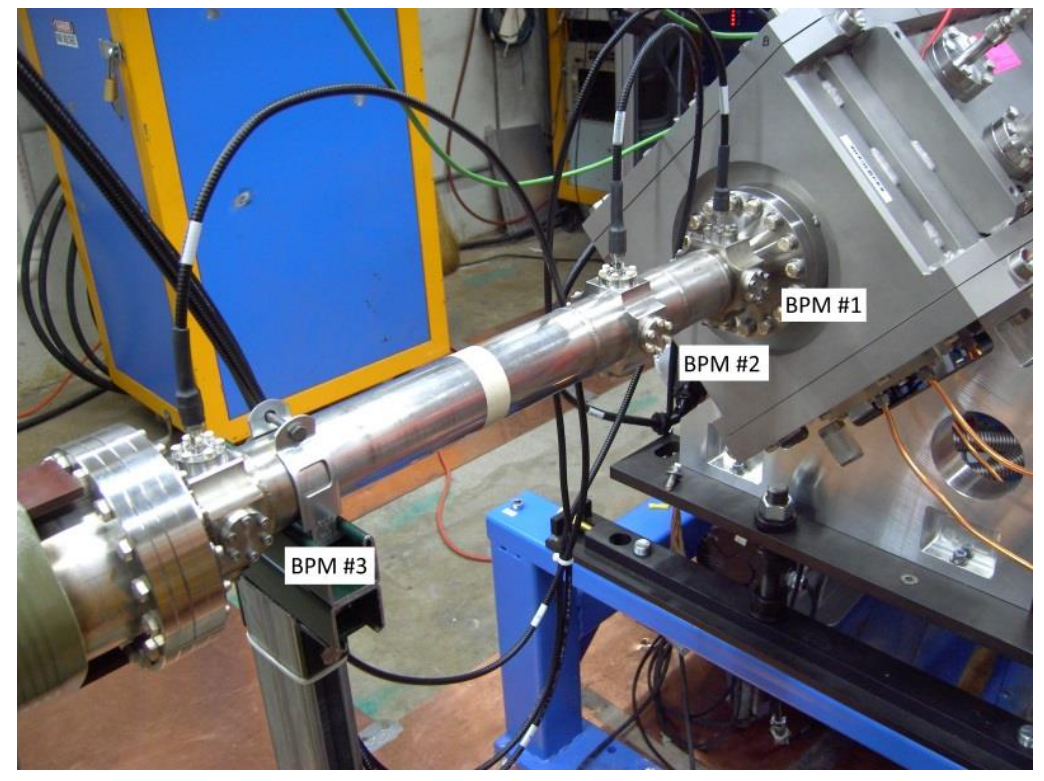

Figure 15: The Time-of-Flight (TOF) experimental setup. The three button type BPMs are labeled as placed downstream of the RFQ.

For each pair, an initial calculation in the time domain using the sequential zero crossings of both BPM signals was made using Mathematica, see [Appendix 3]. The timing of these sequential zero crossings would yield a time span over which the beam pulse travelled between the BPM pair. The beam pulse's velocity is easy to calculate because we know the distance between these BPMs and from this we can extract the $\beta$ and $\gamma$ required to calculate the kinetic 
energy of the beam pulse. This was repeated for all three BPM pairings; 1-2, 2-3, and 1-3. Each measurement showed a beam kinetic energy of approximately $700 \mathrm{keV}$, roughly $50 \mathrm{keV}$ short of the design criterion.

\begin{tabular}{|l|l|l|}
\hline \multicolumn{3}{|c|}{ Table 2 } \\
\hline BPM Pair & Distance (cm) & Energy (keV) \\
\hline $1-2$ & 15.3 & 706 \\
\hline $2-3$ & 40.5 & 707 \\
\hline $1-3$ & 55.8 & 706 \\
\hline
\end{tabular}

Table 2: Table of Tine-of-Flight BPM distances and energy measurements.

The calculations were repeated in the frequency domain to be sure that the sampling of one bunch was not unduly influencing our calculations. This is shown in [Appendix 4]. The waveform data for each BPM was Fourier transformed and the peak location was used to calculate the phase at the $201.25 \mathrm{MHz}$ resonant frequency of the RF. The phase difference between BPM pairs was then used to calculate the beam velocity and thus the beam energy. The kinetic energy was confirmed to be near $700 \mathrm{keV}$.

\subsubsection{Spectrometer Magnet Measurements}

Conferring with the manufacturer on possible causes for the energy error led to a second energy measurement to verify the results of the TOF measurement. Several methods were investigated before the spectrometer magnet was selected because it is the most definitive method for measuring beam energy.

The setup of the spectrometer is shown in Figure 16. Beam exiting the RFQ is first channeled through two narrow slits. These slits were used to establish a known longitudinal axis or zero angle from which to calculate the beam trajectory. After the slits, a dipole spectrometer magnet was set up to bend beam to the right when powered, or straight into a Faraday Cup 
when not powered. Beam was then bent through a separate beam line to a multiwire when the magnet was powered. Upstream of the multiwire is a third slit that was used to establish the trajectory of the beam that hit the multiwire.

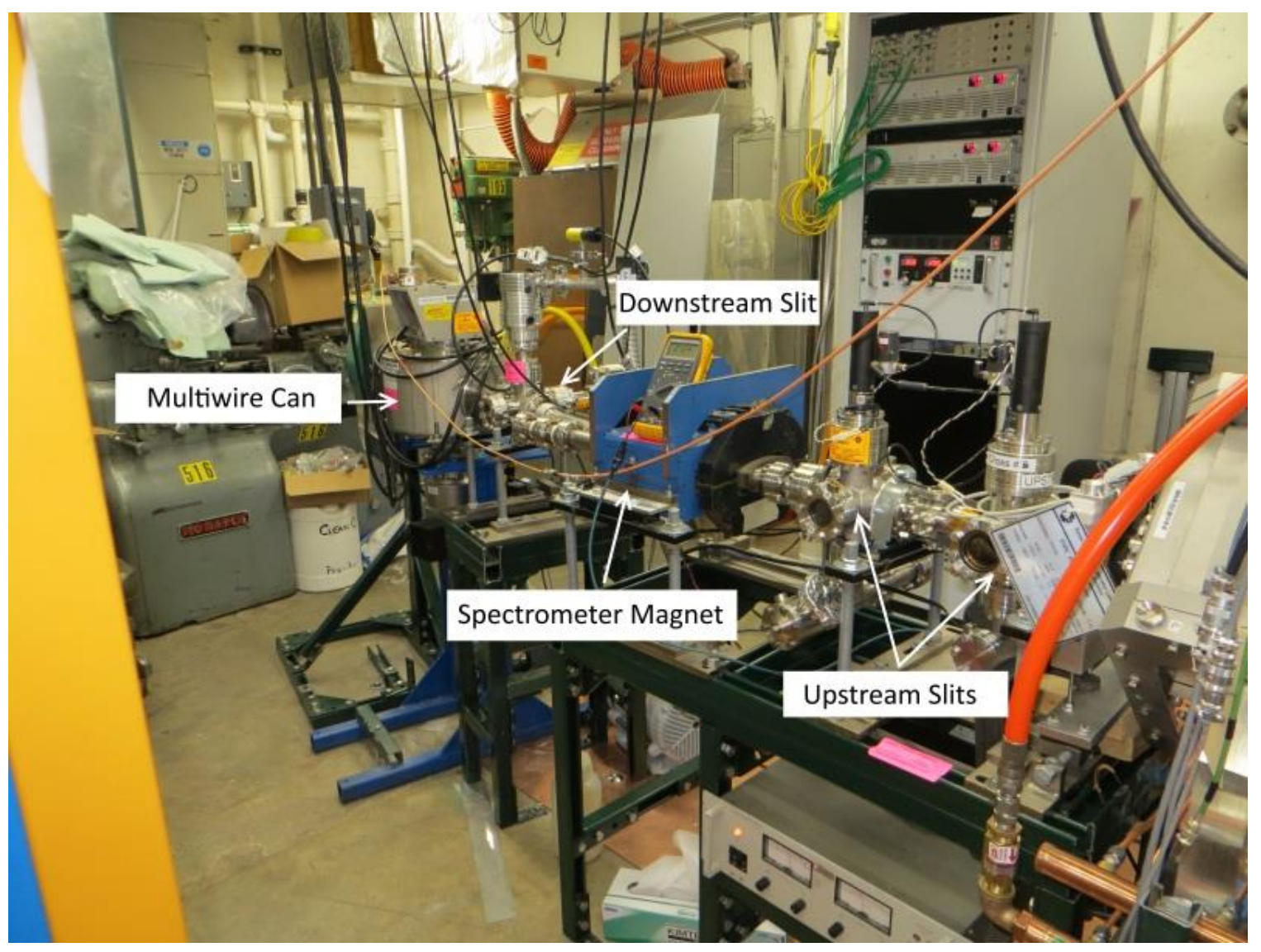

Figure 16: The spectrometer energy measurement setup. The spectrometer magnet bends beam into the multiwire. Slits are used to establish straight lines for easier angle calculations.

The multiwire profiles were then analyzed to find the peak and mean of the signals which were then used to calculate the angle of deflection. This angle and the current in the spectrometer magnet give the beam energy given by Equation 1. $\Theta_{\mathrm{d}}$ is the deflection angle, Bdl is a function of the magnet current measured by the suppliers of the magnet (FNAL Technical Division), and $\mathrm{B} \rho=-\mathrm{p} / \mathrm{c}$, is the beam rigidity. This method confirmed the TOF energy measurement of $700 \mathrm{keV}$. Investigation into the cause of the low energy began while conferring with the manufacturer. 


$$
\theta_{d}=\frac{B d l}{B \rho}
$$

\subsubsection{Energy Error Investigation}

One potential source of the lower than desired kinetic energy was thought to be the RF power going into the RFQ. Initial testing showed that the RFQ required much higher power than was expected. The designed power requirement was for $100 \mathrm{~kW}$ to be enough power to bunch and accelerate the beam in the RFQ to $750 \mathrm{keV}$. However the RFQ required much more power than this for efficient bunching and acceleration. Initial efforts centered on correcting this discrepancy as a possible cause of the low energy output.

Tests were performed to characterize the amount of power required by the RFQ by using the 3 BPM setup from the initial energy measurements and increasing the RFQ power from 110 $\mathrm{kW}$ to $220 \mathrm{~kW}$. The BPM measurements showed that bunching was not evident until at least $130 \mathrm{~kW}$ and was not strong enough to be steadily seen on the $3^{\text {rd }}$ BPM until $180 \mathrm{~kW}$ as seen in Figure 17. The ratio of $2^{\text {nd }} / 1^{\text {st }}$ harmonics for the third BPM takes a step down at this power level. The ratio for the second BPM also takes a step down at this power level. As can be seen in Figure 18 the beam energy cannot be determined with any precision until at least $140 \mathrm{~kW}$ of power is present in the RFQ and the measurements do not agree until over $200 \mathrm{~kW}$. These measurements were verified using the Fast Faraday Cup that was used for the Einzel Lens tests. This device did not show a good bunch structure on the beam until $180 \mathrm{~kW}$.

Upon conferring with the manufacturer the RFQ was opened and the rods were inspected for damage. Scorch marks on the nodes of the rods were evident but were easily cleaned with alcohol and wipes. A survey of the rods also showed two of them to be warped with as big as 1 
$\mathrm{mm}$ deviation from straight. The rods have a minimum aperture of $5 \mathrm{~mm}$ between them through which beam could pass. Aligning the rods to reduce the misalignment did little to correct the energy errors or power coupling into the RFQ.

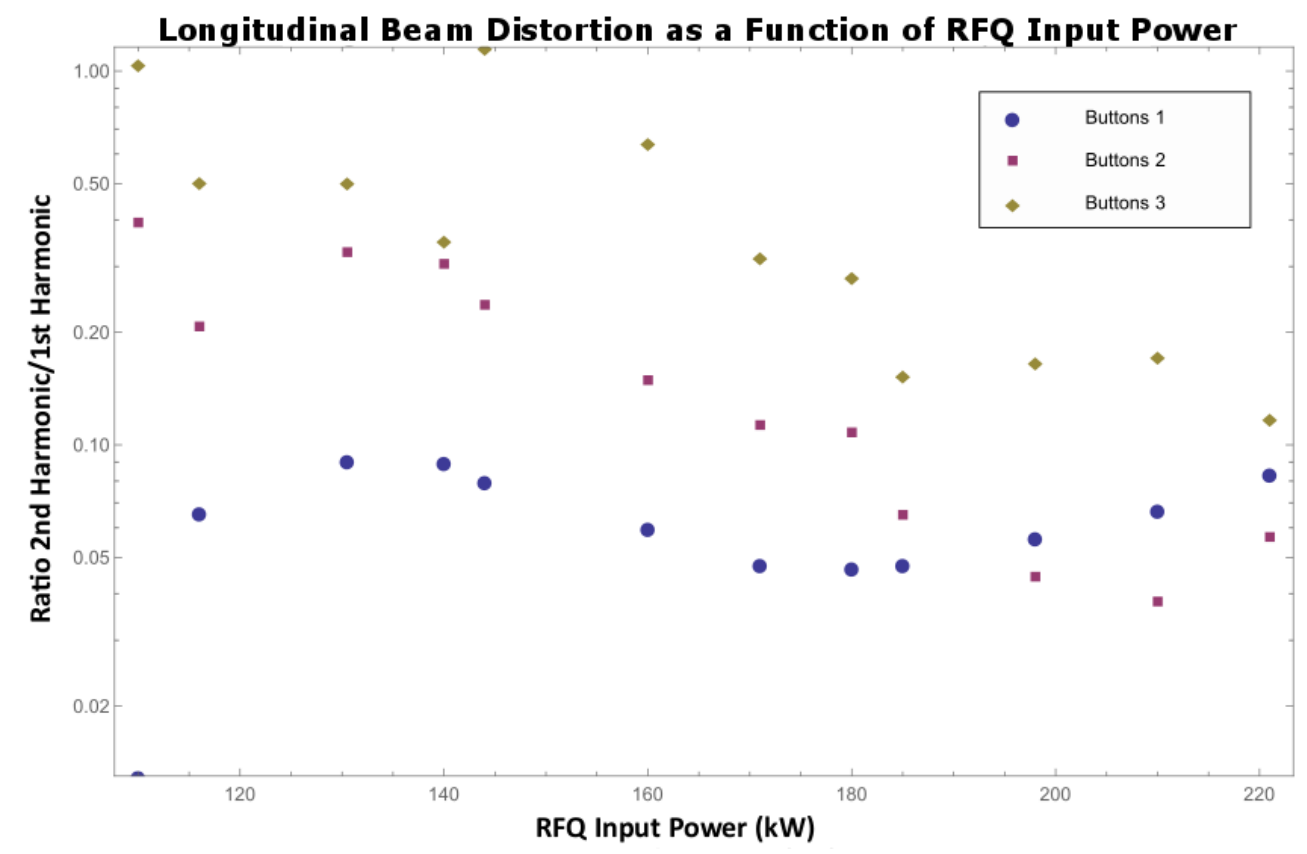

Figure 17: A plot showing the ratio of harmonics in the beam signal as a function of the RFQ input power. Above 180 $\mathrm{kW}$ was required to limit higher harmonics.

Comparing this RFQ with previous models built from the manufacturer showed that the rods in this RFQ were larger transversely than what they had used before. This was done with the intent of making the rods stronger and less susceptible to bending when moved. A new set of rods was ordered and the smaller rods did allow for a lower input power. A visual comparison of the rods can be seen in Figure 19. Bunching was now evident as low as $130 \mathrm{~kW}$ however higher power was still found to produce better bunched beam. Nominal RFQ power for operation is $175 \mathrm{~kW}$ even with the thinner rods.

Simulations performed by S. S. Kurennoy [5] showed that the beam was reaching $750 \mathrm{keV}$ in the RFQ but was slowed down after the beam leaves the rods and before the exit, see Figure 20 . End plates had been installed in the upstream and downstream entrances with $3 / 4$ " apertures to 
limit RF leakage from the RFQ as shown in Figure 21a. Once this end plate was removed from the downstream end of the RFQ, the beam energy from the RFQ was measured to be $757 \mathrm{keV}$ +/- $2.5 \mathrm{keV}$ with $167 \mathrm{~kW}$ of power in the RFQ [6]. The RFQ is operated without this end plate, see Figure 21c.

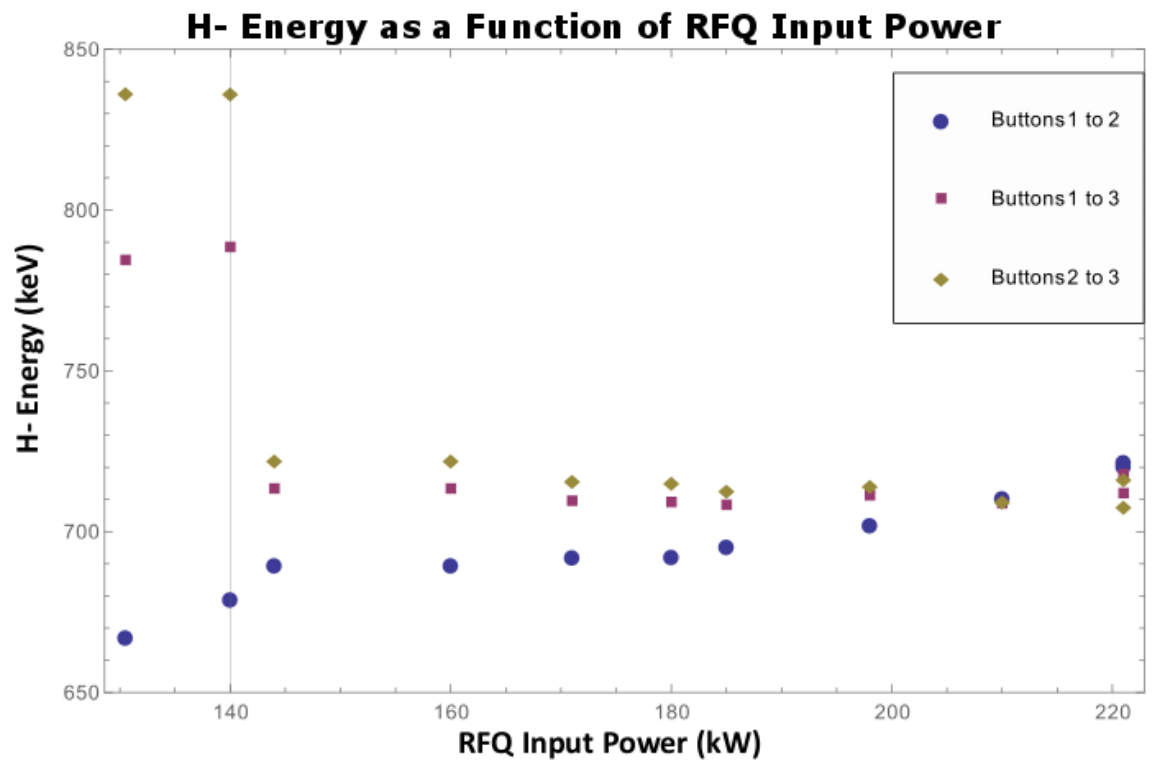

Figure 18: Energy measured as a function of RFQ input power. Reliable values not attainable until $140 \mathrm{~kW}$, and those are not in agreement until over $200 \mathrm{~kW}$.

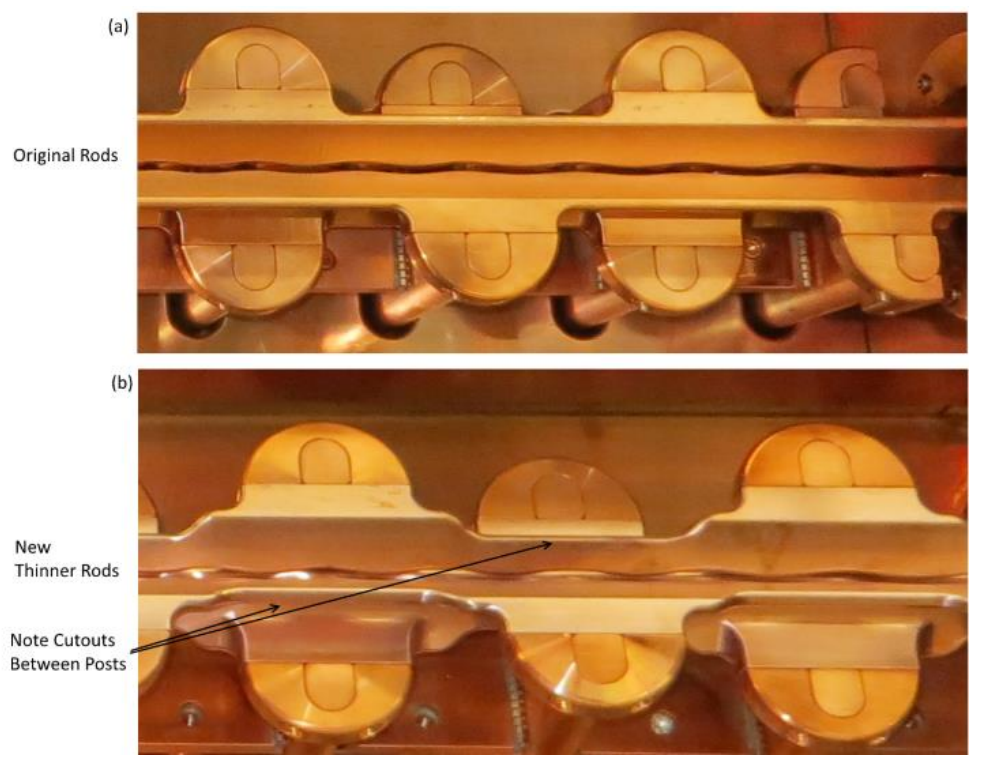

Figure 19: Comparison of two RFQ rod types. The cutouts in (b) reduced the power necessary for beam from over $200 \mathrm{~kW}$ to $140 \mathrm{~kW}$. 


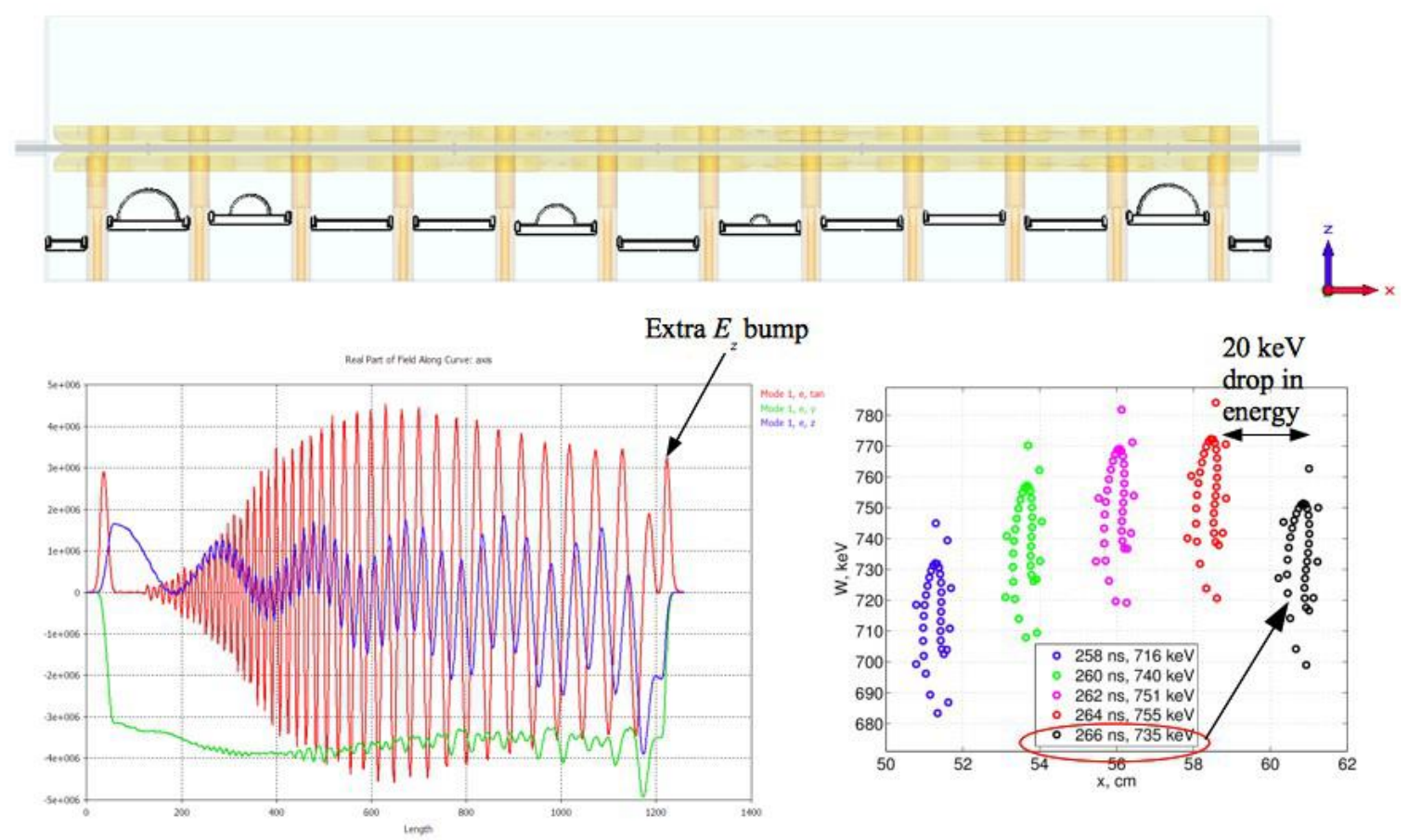

Figure 20: Computer simulations performed by S. Kurennoy of LANL for the RFQ. At the top is a cross section of the model that shows the rods and tuning plates. Some of the plates have half-moon shaped inserts added to help with field flatness. The bottom left picture shows the E-fields along the longitudinal axis of the rods. The red curve is the $E_{z}$ field along this axis. The bottom right picture shows the bunched beam near the RFQ exit. From these pictures it is clear to see that there is a $20 \mathrm{keV}$ drop in beam energy after the ends of the rods and before the exit of the RFQ.

The same simulations that guided us in the removal of the downstream end plate also showed beam would be better served by removing the upstream plate as well. This caused problems with the Einzel Lens that is attached to the RFQ entrance. The Einzel Lens acted as an antenna radiating the $201 \mathrm{MHz}$ RF into the air when the RFQ was operating that caused component trips and sparking in air. A compromise was made by boring out the initial $0.75 \mathrm{in}$. aperture to 1.25 in. as seen in Figure $21 \mathrm{~b}$. This increased the bunching effect of the RFQ while not allowing the $201 \mathrm{MHz}$ RF to leak out and radiate from the Einzel Lens. 


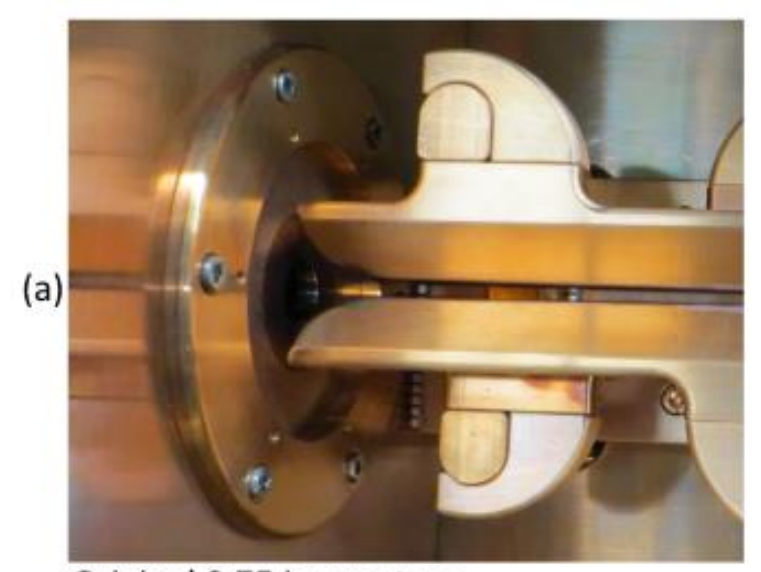

Original 0.75 in aperture

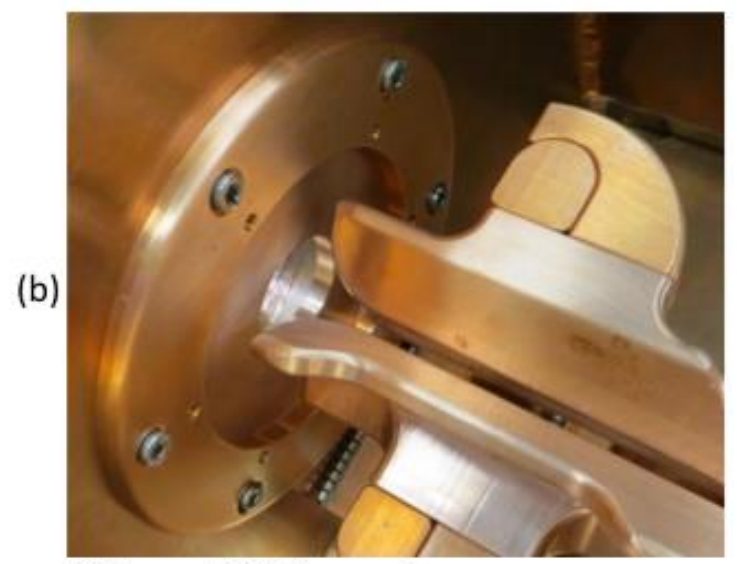

Widened 1.25 in aperture

(c)

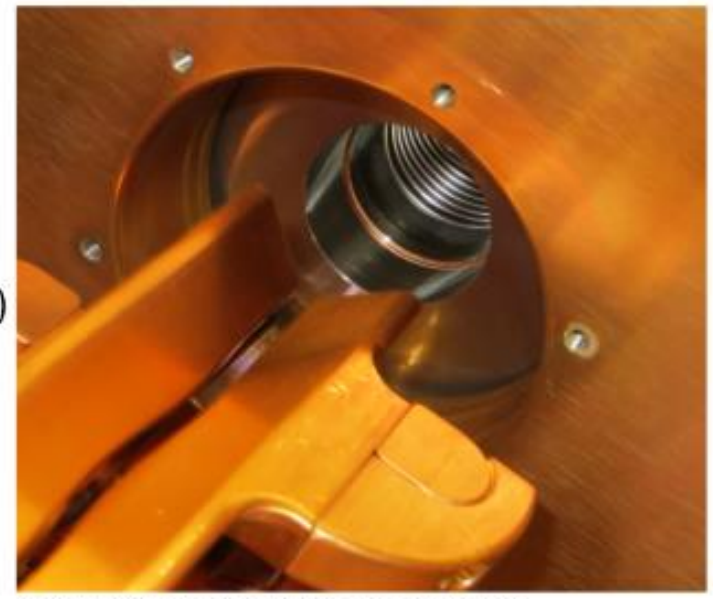

Downstream end plate removed

(screw holes were filled before closing)

Figure 21: RFQ tank end plates from the inside. The 0.75 in aperture was widened to 1.25 in on the upstream side and the entire plate was removed for a 3 in aperture on the downstream end.

\subsection{RFQ Capture Efficiency}

With the energy corrected and RF power issues at least somewhat abated, focus shifted to the RFQ capture efficiency. The design goal of the RFQ called for beam transmission of $>90 \%$ through the RFQ, but even with $200 \mathrm{~kW}$ power in the RFQ it was barely $50 \%$ efficient.

The experimental setup for these measurements included a toroid around a 6" beam pipe attached to the end of the RFQ that terminated in a beam dump. The 6" pipe was used to limit 
beam scraping after it exited the RFQ before reaching the toroid. The beam entering the RFQ was tuned to a focus using the solenoids in the LEBT. The residual gas in the LEBT could also be used to focus the beam once neutralization had taken effect. The gas focusing was found to have a large effect on the beam transmission as was shown in Figure 13.

This was accomplished by slowing the turbo pumps on the LEBT down in discrete steps and monitoring the beam current on the LEBT toroid and the toroid downstream of the RFQ. Using this method, it was found that the optimal LEBT pressure is in the range of $3 \times 10^{-6}$ Torr to $6 \times 10^{-6}$ Torr with the RFQ power near $170 \mathrm{~kW}$. With these parameters $45 \mathrm{~mA}$ was seen at the toroid downstream of the RFQ with $67 \mathrm{~mA}$, an efficiency of $67 \%$. This was not the $90 \%$ that was expected, but it was enough to be able to install the RFQ system to replace the CockcroftWalton system.

\section{Medium Energy Beam Transport}

The Medium Energy Beam Transport (MEBT) is a short beamline that takes the $750 \mathrm{keV}$ beam from the RFQ and matches the beam to the Linac. The first RF cavity in Linac is a drift tube style cavity with quadrupole magnets inside of the drift tubes within the cavity. The Linac tanks are standing wave cavities that require the beam to be shielded from the negative portions of the RF electromagnetic wave, hence the need for the drift tubes. The quadrupoles inside these drift tubes have a repeating structure, or lattice, that requires the beam injected from the RIL to match in order to reduce beam loss. It is also useful to reinforce the $201 \mathrm{MHz} R F$ bunch structure in this beamline as the structure applied from the RFQ will fade quickly after it leaves the RFQ cavity. 
The MEBT consists of two quadrupole doublets with a Buncher RF cavity between them.

Each quadrupole magnet also houses one of each a horizontal and vertical trim making a total of eight dipole trims in the MEBT to go with the four quadrupoles. The MEBT is shown in Figure 22.

The Buncher is a two gap bunching cavity primarily because two single gap cavities would not fit in the space allowed. Grids were inserted into the gap to increase the transit time factor. This was the solution found with the Buncher used at Brookhaven National Laboratory [7]. The grids prevent the electrical field from leaking past the physical gap thus creating a longer gap length. A longer gap length leads to a smaller transit time factor, which was confirmed with a bead pull measurement.

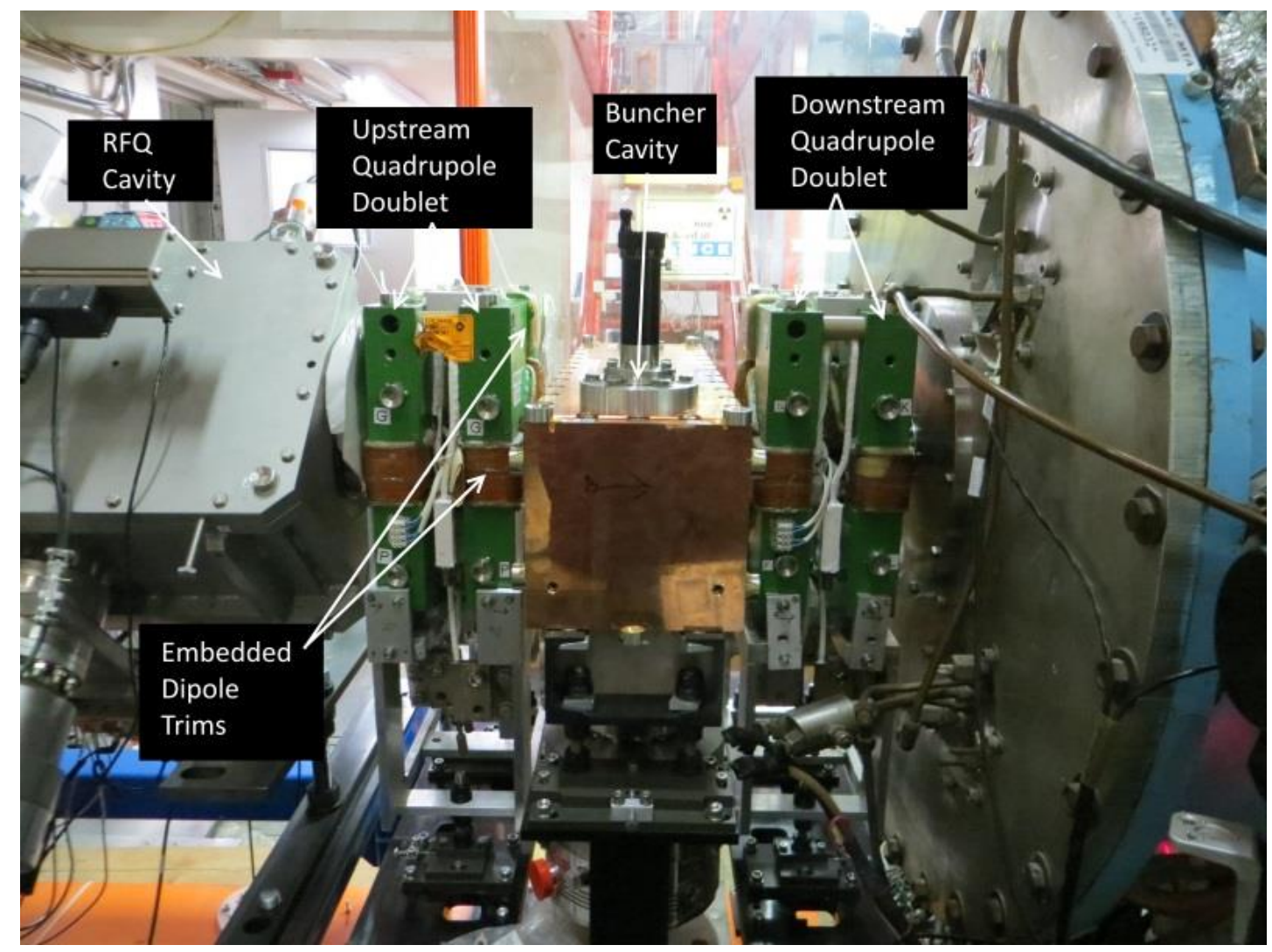

Figure 22: The RIL MEBT with the two quadrupole doublets and a two gap Buncher cavity. 
The Buncher was low power conditioned the same way as the RFQ. The cavity was placed under vacuum and low power, up to $70 \mathrm{~W}$ CW, was provided to the cavity. Initial problems with a loose input connector and a dirty and loose input coupler were fixed. During a break in the RFQ commissioning, the RF amplification system for the RFQ was used to high power condition the Buncher to $3 \mathrm{~kW}$ pulsed for a period of two weeks. Over this time the vacuum pressure in the cavity continued to improve with little sparking in the cavity. It was now considered ready for installation with the rest of the MEBT.

The test area where these systems were commissioned did not have a way to deliver RF power to the Buncher and the RFQ simultaneously, nor did it have the capabilities to power and cool the four quadrupoles. As a result the commissioning of the complete MEBT had to be done in situ after the assembled Source, LEBT and RFQ were installed ahead of Tank 1. Beam was verified at the end of the RFQ after it was installed ahead of Linac, and then the MEBT was installed and commissioned using the emittance scanner and toroid at the beginning of Linac Tank 1.

Initially the intensity to Linac was limited to a little over $30 \mathrm{~mA}$, far below the $45 \mathrm{~mA}$ seen in the test area. The Einzel lens while physically in place was not powered yet during these initial tests, and this did cause a $10 \%$ hit to the intensity. Without the Einzel Lens to chop the beam, the beam pulse length was set by using the RFQ RF pulse as the leading edge and the turning off of the Source arc as the trailing edge. Once the Einzel Lens switches were installed and were able to pulse the lens, the intensity into Linac rose to $33.6 \mathrm{~mA}$. The rise and fall times of the Einzel Lens are short compared to the RFQ RF pulse so less of the beam pulse was affected during the RF power ramp. There is also likely some small amount of steering of the beam even when the lens is between pulses. 
The RFQ power needed to be held around $170 \mathrm{~kW}$ or greater for increased beam into Linac as well. Losing even 10 to $15 \mathrm{~kW}$ in RF power caused a drop in efficiency of $10 \%$, roughly what was gained by using the Einzel Lens. Even today the RFQ power is kept above $175 \mathrm{~kW}$ to ensure high transmission into Linac.

It was discovered that the beam exits the RFQ with a sharp upward angle. The first vertical trim downstream of the RFQ on the first MEBT quad assembly had to be run as high current as the design would allow, $-4 \mathrm{~A}$. The trim is able to correct roughly 1 degree of angle but even with this bend $25 \%$ of the beam exiting the RFQ is lost before it gets to Linac. The RFQ and MEBT were realigned to try to reduce the power needed by the first trim but to no avail. A new thin profile vertical trim was placed between the RFQ and the first quad magnet that would allow for an extra vertical kick closer to the exit of the RFQ. $40 \mathrm{~mA}$ was achieved into Linac with this trim shown in Figure 23 and some retuning of the RIL. 


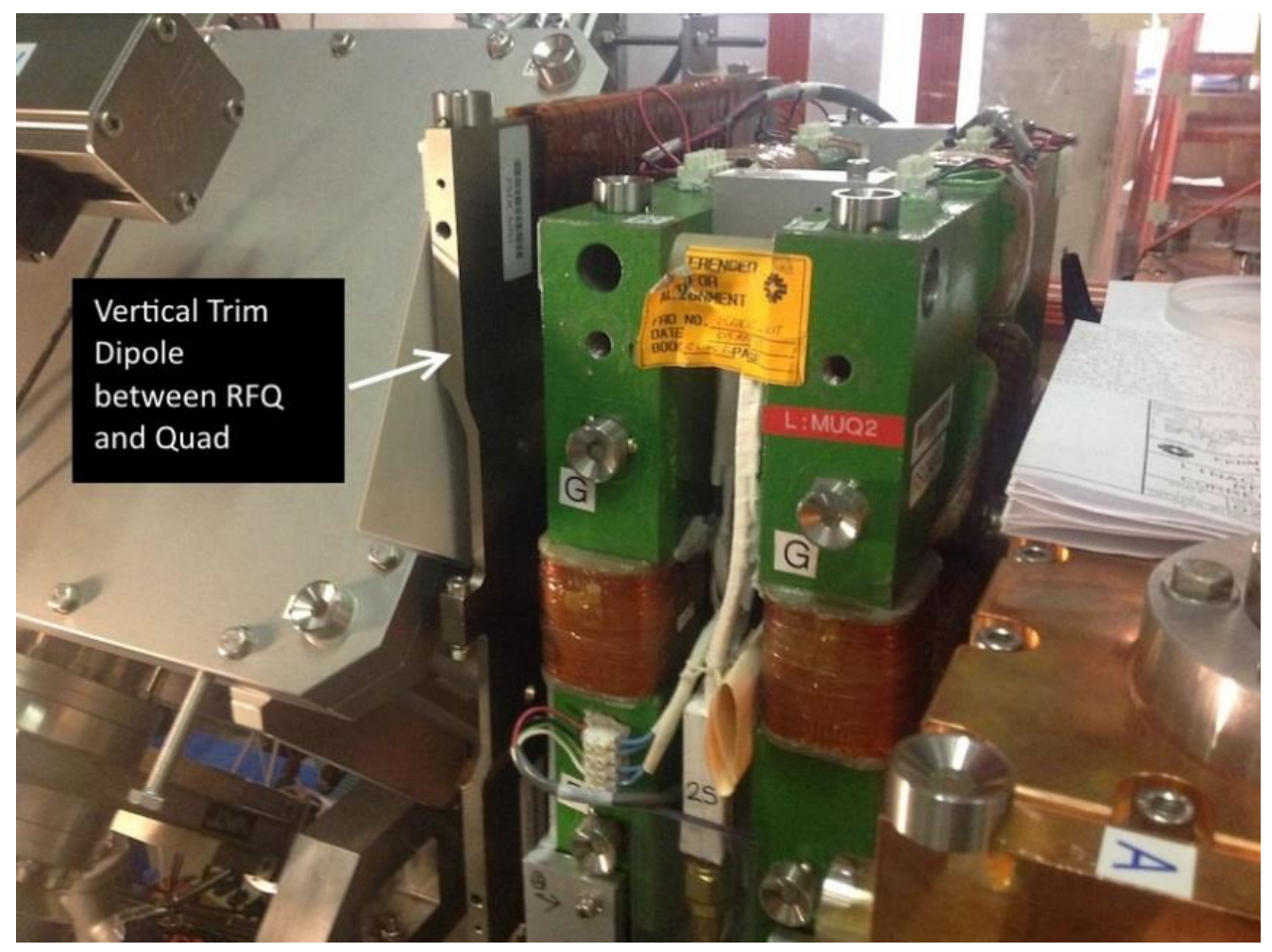

Figure 23: A dipole trim magnet was designed to fit in the small space between the RFQ and first MEBT quadrupole.

When tuned for maximum transmission through the RIL, the transverse emittances are $\varepsilon_{\mathrm{x}}=$ $0.440 \pi \mathrm{mm}$ mrad and $\varepsilon_{\mathrm{y}}=0.5131 \pi \mathrm{mm}$ mrad. Unfortunately when tuned for maximum intensity in the RIL, beam is not well matched to Linac. Current nominal beam current into Linac falls between 25 and $30 \mathrm{~mA}$ with emittances of $0.6263 \pi \mathrm{mm}$-mrad horizontal and $0.4761 \pi \mathrm{mm}$ mrad vertical. While this reduces the overall transmission efficiency of the RIL to $\sim 50 \%$ it helps to increase the Linac efficiency to above $92 \%$. This is a great improvement over the previous Linac efficiency of $74 \%$. It is better to lose the beam at lower energies of $35 \mathrm{keV}$, or even 750 keV. This low energy $\mathrm{H}^{-}$beam will not create radioactivity as readily as a $(22-400) \mathrm{MeV} \mathrm{H}^{-}$ beam will. The lower intensity into Linac is a tradeoff for the much higher transmission efficiency and much lower high energy losses through $400 \mathrm{MeV}$. 


\section{Ion Source}

The ion source, as the name suggests, is the beginning of the RIL and where the $\mathrm{H}^{-}$ion beam is created. The particular style of ion source used in both the Cockcroft Walton as well as the RIL is the magnetron source.

A FNAL magnetron source has two electrodes with a gap between them where hydrogen is injected. Once the hydrogen fills this region a potential is drawn across the electrodes. This creates a plasma where $\mathrm{H}^{-}$ions are created via interactions of the gas molecules with one another, via free electrons, and via interactions with the electrode surfaces. Cesium is used to cover the electrodes, to increase the number of free electrons available to the plasma for ion creation. Magnets are used to confine the free electrons within this plasma, increasing their likelihood of being attached to a hydrogen molecule, thereby creating an $\mathrm{H}^{-}$ion.

This $\mathrm{H}^{-}$ion rich plasma is then exposed to a larger potential difference near the extraction aperture. This extraction potential draws the negative ions as well as free electrons out through the extraction aperture and into the beamline. The magnets used to confine the plasma also cause the co-extracted electrons to be bent away from the beamline, allowing only the heavier $\mathrm{H}^{-}$ions to continue straight into the beamline. In the case of the RIL, into the LEBT where it is focused and transferred to the RFQ.

The choice to continue with the magnetron style $\mathrm{H}^{-}$ion sources was made to capitalize on the 40 year history of operating these sources for the FNAL HEP program. The sources had run reliably and dependably over those 40 years and with relatively minor changes could be redesigned to work with the new front end to provide $\mathrm{H}^{-}$ions for many more years. The retirement of the personnel who had accumulated the 40 years of operational knowledge required the re-learning of many of the operational details of magnetron sources while adapting 
this knowledge to the modifications that were made for the RIL Ion Sources [8]. The magnetron underwent many changes as it was adapted to the RIL system; some small, some significantly larger.

\subsection{Cathode Surface}

A seemingly minor change was the design of the cathode shape, specifically on the side of the cathode that faced the extraction channel. The original cathode used in the early FNAL magnetrons had a flat surface. This evolved over the years to the grooved surface shown in Figure 24. This groove was designed to focus the surface created ions to the extraction channel. The extraction hole in the anode was a slit $1 \mathrm{~mm}$ by $10 \mathrm{~mm}$ to allow for maximum beam extraction.

The re-design for the RIL was needed to address the non-round beam that was created by the previous sources. To create a round beam, a circular dimple shape in the cathode was needed as shown in Figure $24 \mathrm{~d}$. The circular symmetry of this dimple is used to create a round beam instead of the ribbon beam extracted from the slit of the previous sources. This did reduce the surface area of the cathode oriented at the extraction aperture however this was compensated for with the higher extraction voltage in pulling more ions from the source. A perfectly round beam was not seen on the emittance scans in the LEBT and RFQ accelerated beam. This was found to be caused by the dipole field of the permanent magnets affecting the $H^{-}$beam exiting the source, and will be discussed in the Magnetic Field Section [5.3.2]. 


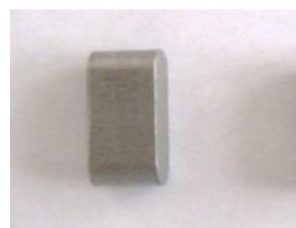

a)

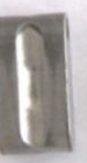

b)

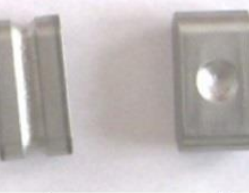

c) d)

Figure 24: Evolution of FNAL magnetron cathodes. The first cathode (a) was flat. A groove facing the extraction slit was added in (b). This groove was extended all-around in (c), and (d) shows the new dimpled cathode design.

The extractor provided the initial acceleration in the old sources with a typical value near 18 $\mathrm{kV}$ applied to the extractor. This was then accelerated through the column to $750 \mathrm{keV}$. The new sources would again have only the extractor to provide initial acceleration for the $\mathrm{H}^{-}$beam before it reached the RFQ, so the extractor would have to run at $35 \mathrm{kV}$ to create the $35 \mathrm{keV} \mathrm{H}^{-}$ beam. This higher extraction voltage pulled more $\mathrm{H}^{-}$ions from the source increasing the beam current while simultaneously allowing the source arc discharge current to run at a much lower value. To provide $40 \mathrm{~mA}$ of beam to the Linac, the old sources required an arc discharge current of $50 \mathrm{~A}$. The new sources with the higher extraction voltage can produce a similar beam current with only $15 \mathrm{~A}$ of arc discharge current.

This lower arc discharge current also allows for a much slower consumption of cesium that is continuously used to cover the surface of the cathode to lower the work function of the molybdenum cathode [9]. A $5 \mathrm{~g}$ ampule of cesium has been shown to last for nearly 600 days, compared to 400 days for the old sources. The estimated lifetime of this magnetron design is 9 months with the lower cesium burn rate and lower arc discharge current. The current longest operational lifetime for one source has reached 8 months and counting (as of June 2015). 


\subsection{Extraction Design Changes}

One of the larger changes was the style of extraction of the ions from the source. The old system had the source situated such that it extracted vertically downwards toward the floor and bent $90^{\circ}$ toward the acceleration column and the wall toward the Linac. This is shown in Figure 25.

The extraction plate sat $2 \mathrm{~mm}$ below the source body extraction slit and was pulsed to approximately $18 \mathrm{kV}$. The $\mathrm{H}^{-}$beam was highly divergent due to the high space charge forces inherent to this type of source as well as the defocusing effects of the extractor plate.

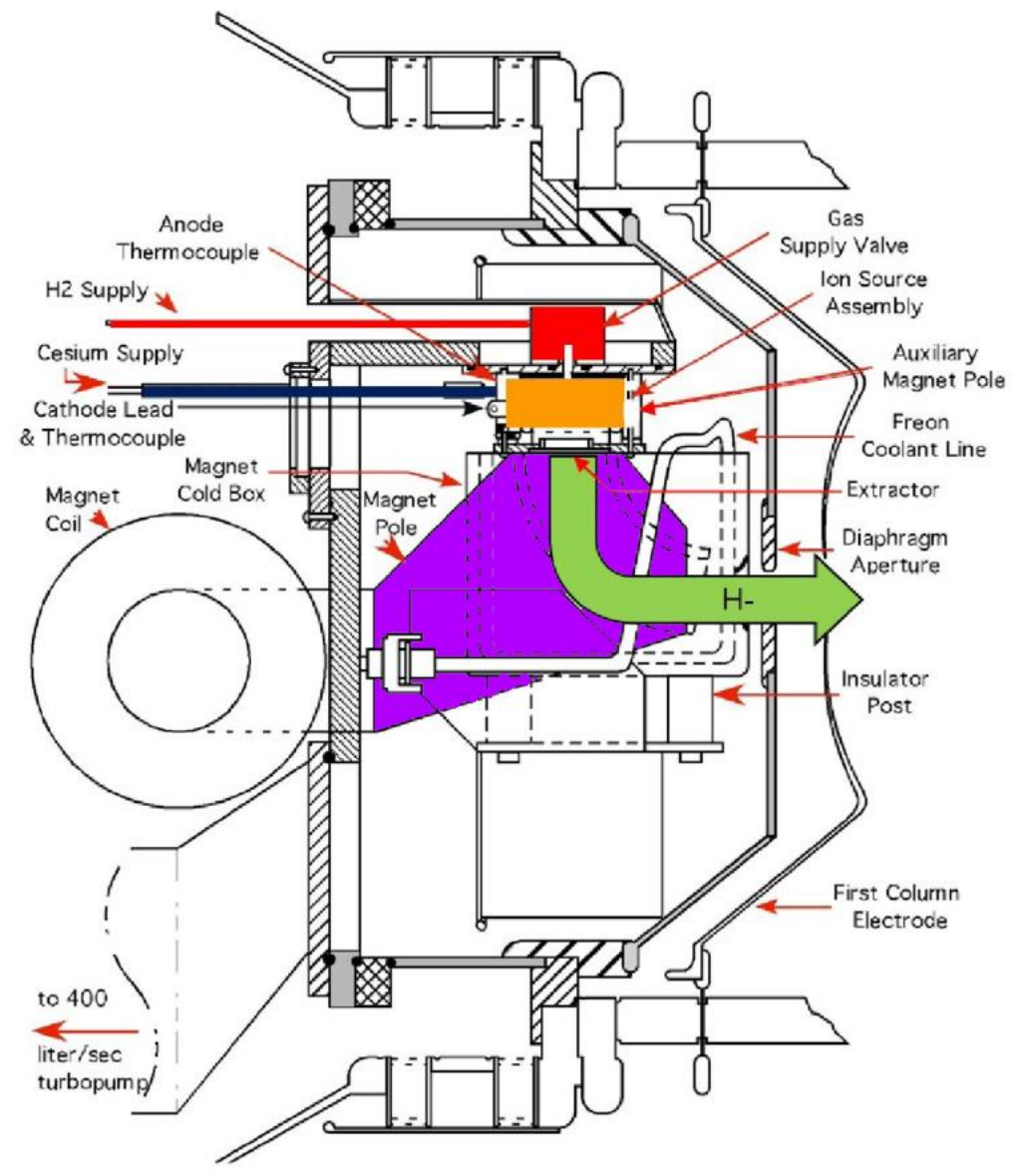

Figure 25: The old source diagram with the hydrogen path in red, cesium in blue, magnet poles in purple, and $\mathrm{H}^{-}$ ion beam in green. 
This source magnet shown in Figure 25 was a dipole with pole faces on both sides of the extractor plate extending down to bend the beam $90^{\circ}$ into the acceleration column. The pole faces were slanted to provide focusing as well as bending of the beam into the column.

Pole tip extensions of the magnet extended upwards to cover the source body and provide the magnetic field necessary to sustain the plasma in the source. It also provided a way to remove co-extracted electrons from the $\mathrm{H}^{-}$beam as it exited the source. The electrons were bent to a much sharper angle relative to the $\mathrm{H}^{-}$ions and did not go into the accelerating column. A cold box below the extractor was cooled to $-30^{\circ} \mathrm{C}$ to capture cesium atoms that escape from the source before it reached the accelerating column. This $90^{\circ}$ bend was made along the long dimension of the extraction slit and resulted in a larger vertical emittance than the horizontal as beam entered Linac.

\subsubsection{New Magnetron Extraction Design}

The new extraction scheme is shown in Figure 26. Beam is extracted straight from the cathode through the extraction aperture. It then proceeds straight across the extraction gap where the $35 \mathrm{kV}$ potential is applied to accelerate the beam into the LEBT. The $90^{\circ}$ bend is removed entirely.

The cold box is no longer used. There is less concern for the cesium bleeding out of the source due to the lower burn rate with these sources so a cold box is not required. The powered magnet poles have been replaced with permanent magnets around the source. This was done to reduce the number of vacuum feed-thru ports on the source cube. 


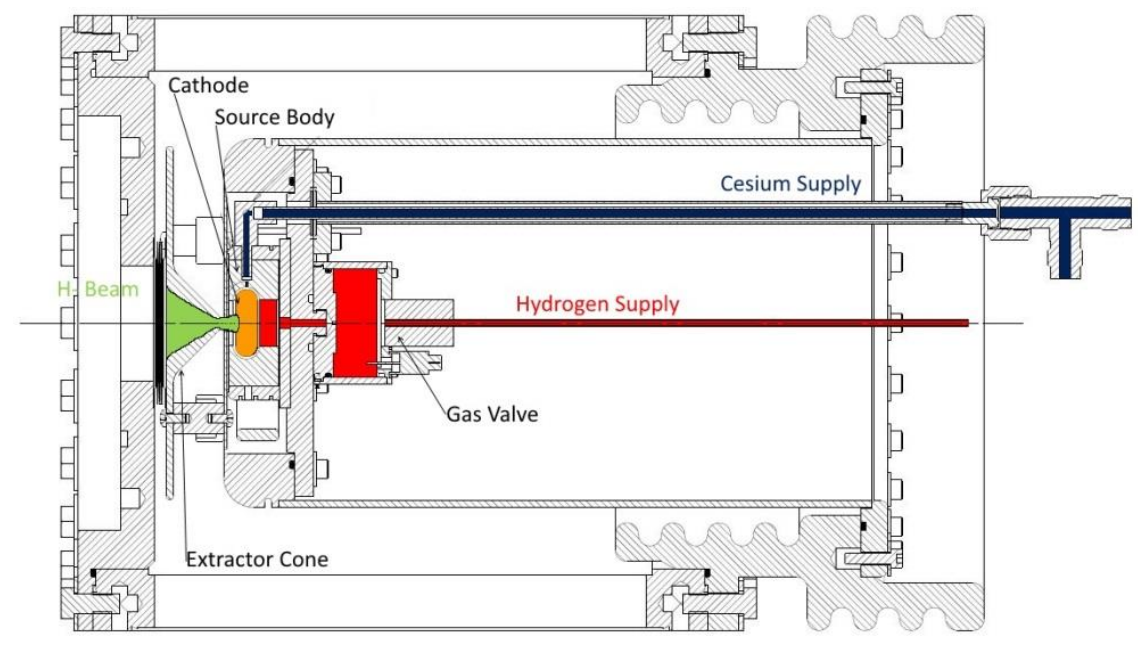

Figure 26: The new source design. The source is mounted on a long can reentrant to the source cube. Extraction is direct from the cathode with no bending magnet. Hydrogen flow is in red, cesium in blue, cathode in orange, and $\mathrm{H}$ beam flow is in green.

The shape of the extractor plate was modified into a cone to limit the surface area that would come in to close proximity of the source body. The extractor plate is attached to the source can with three ceramic standoffs. During the extraction pulse, the two have a potential difference of $35 \mathrm{kV}$. Initial tests to pulse the extractor plate to $+35 \mathrm{kV}$ were abandoned due to sparking and problems with the feed-thru in the source can. Alternatively, the entire can, source body, power supplies, and electronics are pulsed to $-35 \mathrm{kV}$ while the extractor plate is connected to ground potential at the back wall of the source cube. After considerable time and effort this design has led to a source that averages as few as one extractor to source spark a day.

A simple electrical block diagram showing the basic design of three different types of pulsers are shown in Figure 27. (a) shows the design used in the Cockcroft Walton sources where only $18 \mathrm{kV}$ were needed for extraction. These pulsers used a positive voltage because the source was pulsed to $-750 \mathrm{kV}$ with the rest of the dome and it needed the positive voltage to attract the $\mathrm{H}^{-}$ions. (b) shows the pulser for the RIL source. This pulsed to $-35 \mathrm{kV}$ because it was 
pulsing the source body as the extractor cone sits at ground potential. In both (a) and (b) the screen is held at $+400 \mathrm{~V}$ while the control grid is pulsed from $-200 \mathrm{~V}$ to $0 \mathrm{~V}$ to trigger the tube to conduct current during the pulse. The rise time of this varies, for example a brand new tube has a rise time of over $100 \mu \mathrm{s}$. This circuit in (b) has been replaced by solid state switches that are just told to close during the pulse in (c). This solid state system is discussed in greater detail in Section 5.4.5.

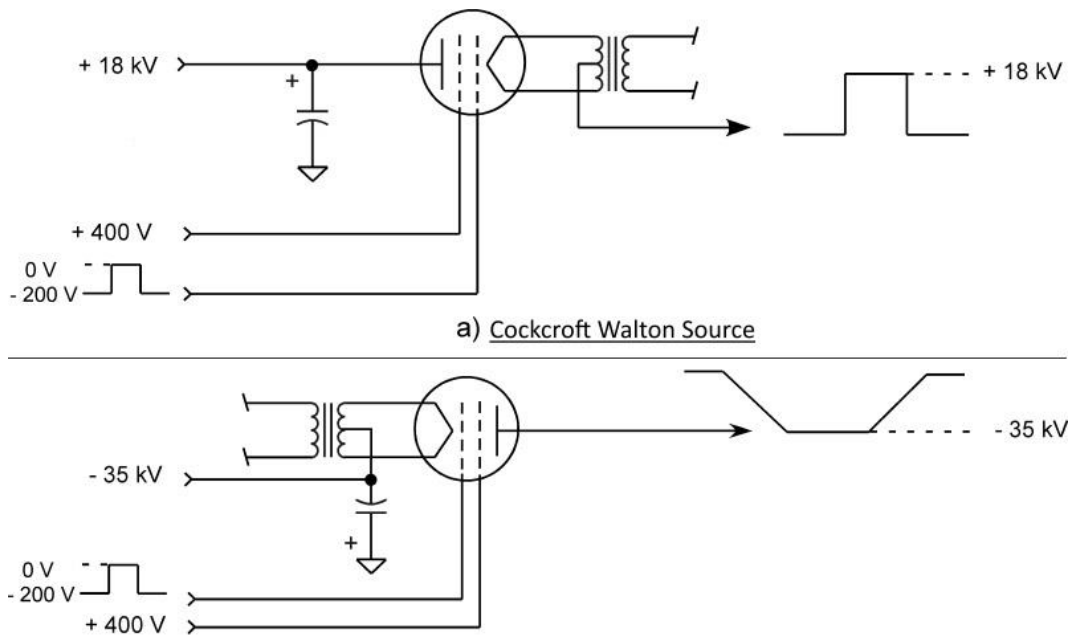

b) RIL Source Tube Pulser

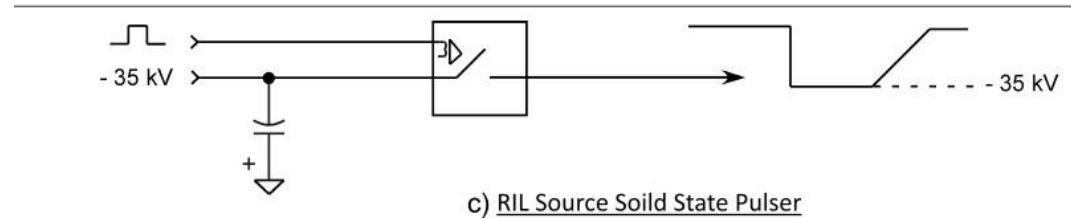

Figure 27: Simple electrical diagrams for the extractor pulser. The C-W sources only pulsed to $+18 \mathrm{kV}(\mathrm{a})$, while the new RIL source pulses to $-35 \mathrm{kV}(\mathrm{b})$, and the solid state system currently used (c).

\subsection{Extractor Spark Mitigation}

Sparking that occurs between the extractor cone and the source can has the potential to be very damaging to the operation of the source. The source electronics that are present in the high voltage rack that are pulsed to $-35 \mathrm{kV}$ are quite susceptible to ground loop problems and errant pulses. Specifically the Hotlink Rack Monitor (HRM) that handles communication 
between the controls system outside of the HV rack and within the rack was frequently affected by extractor sparking. A voltage spike would cause the HRM to set the arc power supply and source heater power supplies to zero output until the HRM was reset.

The source heater power supplies are necessary for keeping the cesium system at a high enough temperature to keep cesium flowing to the source as well as maintaining a high enough source body temperature to maintain the plasma in the source. When these are turned off, the source would falter and the plasma would die out. If the arc power supply was set to zero output, then the arc discharge would never ignite the plasma for the beam pulse. If this happened for more than a few beam cycles, the source temperatures would cool off thus affecting the source operation. Efforts to remove the HRM from the high voltage rack with the use of fiber optic relay channels to communicate with the source look promising. Even so, these units are still susceptible to the effects of extractor sparking. Keeping the source from sparking in the first place is critically important and has required several changes to the initial design, such as the choice of materials for the source body and extractor cone.

\subsubsection{Materials for Source Components}

The new source body design borrowed as much as possible from the original FNAL magnetron design by C.W. Schmidt [1] to simplify operational changes. The parts of the old magnetron are shown in Figure 28. The materials in close proximity to the plasma have to be able to withstand erosion from the plasma as well as the high temperatures near the plasma. This made molybdenum the metal of choice for the extractor cone tip, anode, and cathode. 


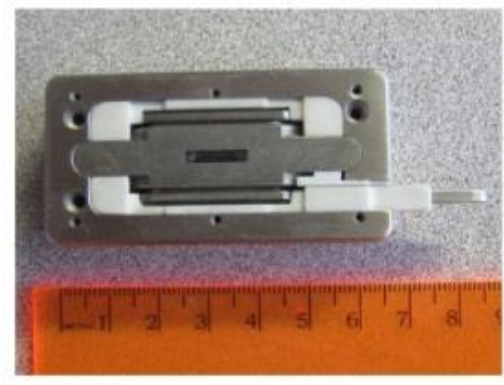

a)

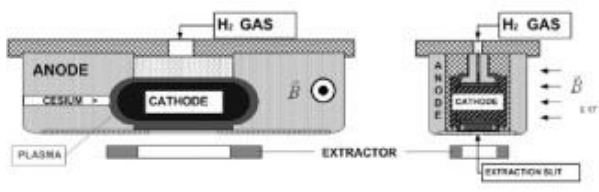

b)

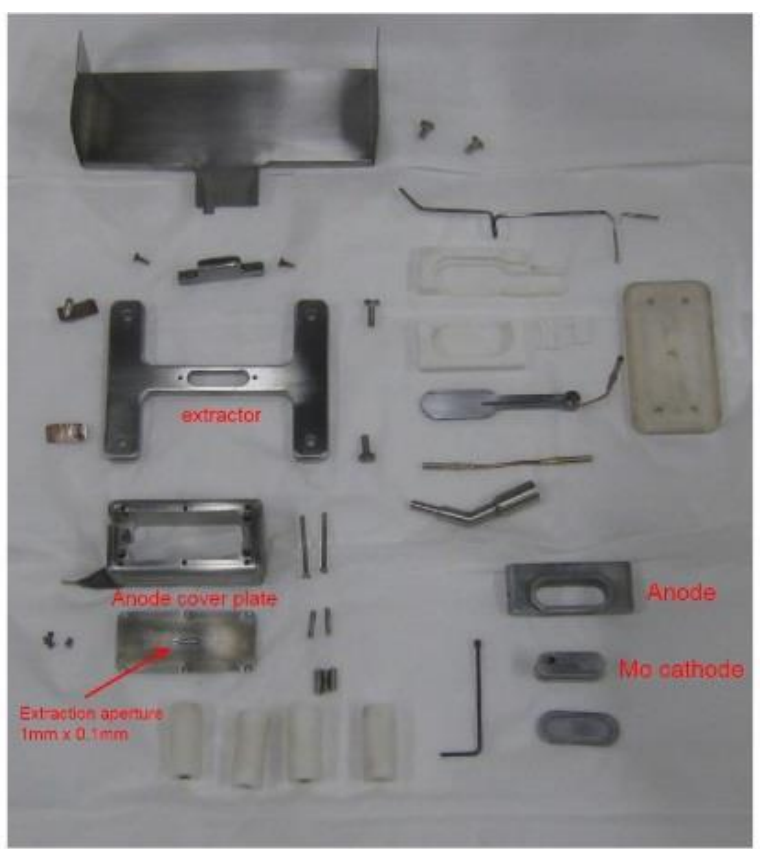

c)

Figure 28: The old C-W style magnetron source assembled (a) and disassembled in (c). Diagram in (b) shows the various inputs and output.

The extractor cone tip was still damaged by sparking as shown in Figure 29a, even though it is made out of molybdenum. Repeated sparks from the anode cover plate to the extractor cone tip would lead to sharp points developing on the cone tip. These sharp points would then become antennas that became sources for continuous sparking. This damage would require the source to be removed so that these points could be sanded down and refinished or the cone tip replaced entirely. Other damage was caused by the stream of co-extracted electrons as seen in Figure 29b. The electrons are diverted by the magnetic field of the permanent magnets and over time would cut a notch into the extractor cone tip. After consulting with experts from BNL, the design was altered to allow for a tungsten insert into the molybdenum cone tip as shown in Figure 29c. Tungsten is a harder metal and the new inserts have shown little damage from sparking across the extraction gap. 


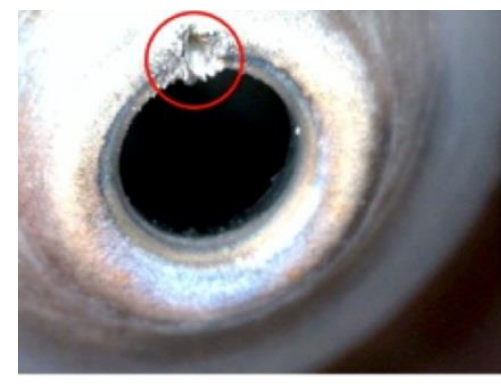

a)

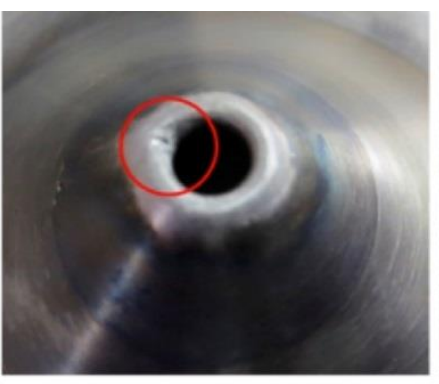

b)

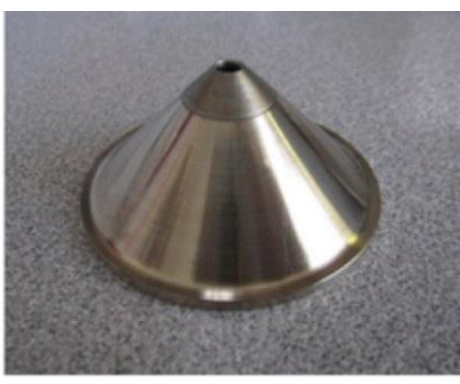

c)

Figure 29: The RIL source extraction cone. Damage to the cone tip by repeated sparking is shown in (a) and electron bombardment in (b). The new tungsten tip inset in the cone is shown in (c).

On the upstream side of that extraction gap, on the source side, is the anode cover plate. This plate has the extraction gap on one side and the plasma gap on the other. It is a point where sparking and erosion could both cause problems at the circular aperture. Originally this was made from titanium. These plates would suffer erosion from the plasma and from the ions streaming through it as shown in Figure 30. This erosion would occur unevenly resulting in a less transversely round beam, but more importantly it would also create sharp points that would lead to more sparking across the extraction gap. A change to a molybdenum plate still showed signs of erosion, which like the extractor cone tip, had led to a tungsten anode cover plate being used. These plates made from tungsten are now standard, and similar to the extractor cone tip, show few signs of damage.

The source cathode has long been made of molybdenum due to its durability and low work function that makes it a great electron provider for the plasma, especially when coated appropriately with cesium [9]. Even with erosion evident from the plasma, molybdenum continues to be used to create high intensity $\mathrm{H}^{-}$beams. Tungsten would be a possible replacement metal for the cathode. It has a higher work function and lower $\mathrm{H}^{-}$yield than molybdenum but it does have a broader peak for $\mathrm{H}^{-}$production which might allow for more 
flexibility in source parameters. A plan to create a molybdenum cathode body with a tungsten cathode dimple insert is afoot but at present has not been made into a reality.

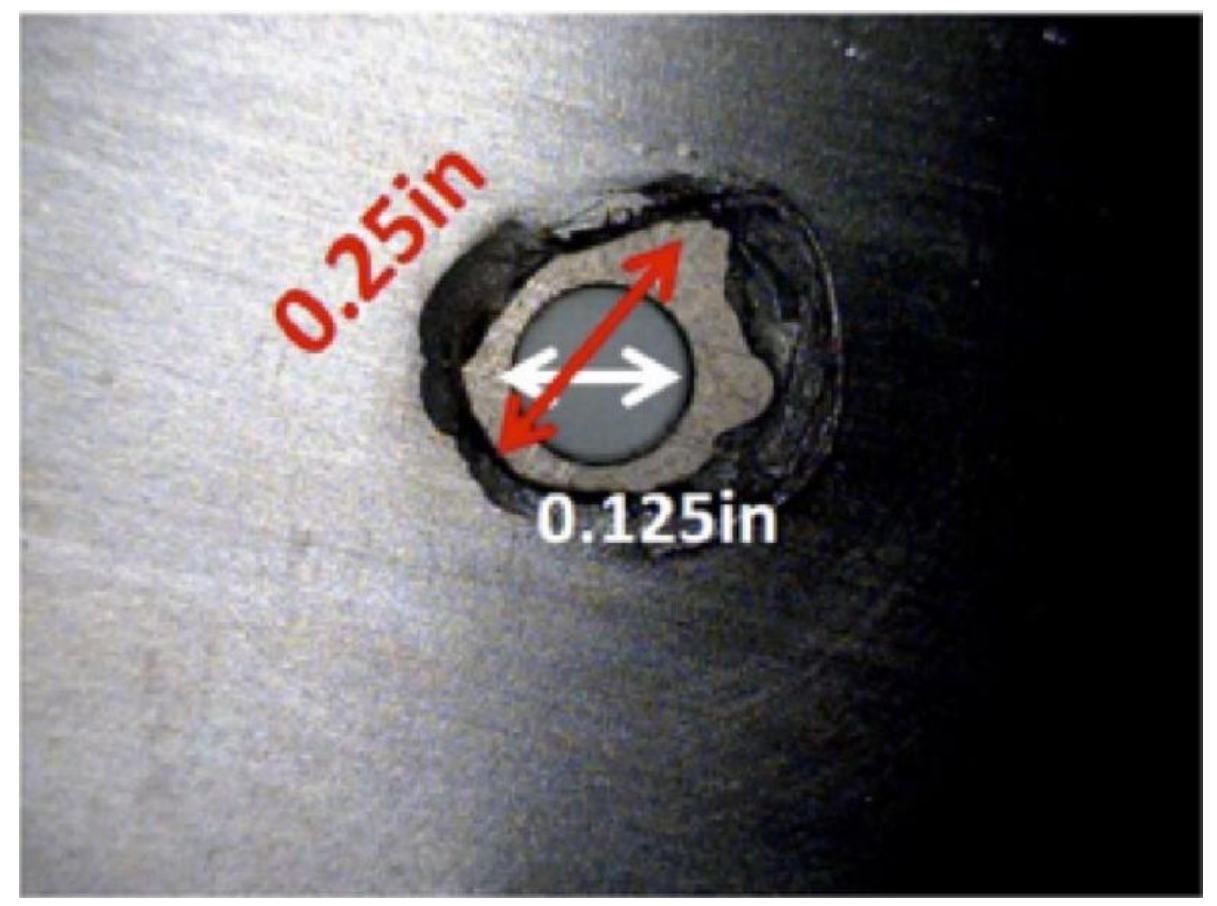

Figure 30: Damage to anode cover plate shown here can lead to sparking in the extraction gap even as it allows more beam through the enlarged aperture.

\subsubsection{Source Magnetic Field}

A magnetic field is critically important for confining the plasma and electrons within a magnetron source. A magnetic field transverse to the beam propagation direction is used to confine the electrons within the plasma. Too weak of a magnetic field within the plasma, and the electrons will be lost to the high electric field of the extractor. Simulations showed that a transverse magnetic field strength of $1 \mathrm{kG}$ was necessary to limit electron loss to a level that allowed for sustained plasma [8]. This also serves to limit the co-extracted electrons that can cause extraction gap sparking. 
The direct extraction scheme for the new sources no longer required the magnet to bend the beam into the accelerating column. It was needed only to provide the field for the source plasma. As a result, permanent magnets were chosen to simplify the design because no feedthru was needed to power the magnets.

The original design for these source magnets used four disc shaped permanent magnets mounted on a U-shaped iron yoke. See Figure 31a. The commercially available 3/4" diameter, 1/4" thick samarium cobalt permanent magnets are mounted two to a side on the ends of the yoke to go around the source body. Thick steel back plates were used to keep the magnets close to the source and steel pole tips were placed between the source and the magnets. Unfortunately this design did not create a strong enough magnetic field in the plasma region and excessive sparking in the extractor gap resulted. The second attempt doubled the magnets to four a side, giving eight in total. The thick back plate was removed to make room for the extra magnets. See Figure 31b. This did improve the magnetic field, but not quite enough to get $1 \mathrm{kG}$ in the plasma region.

The third attempt involved a redesign of the magnets and the yoke seen in Figure 31c. The yoke was made thicker and taller to enable it to channel more magnetic field flux. The 4 circular magnets per side were replaced with 1 rectangular magnet, still made of samarium cobalt. This design increased the field in the plasma region as well as provided a stronger bend to the lessened co-extracted electron beam to prevent it from striking the extractor cone tip. Figure 32 shows a comparison of the magnetic field strength overlaid atop the source geometry. With this new design the field in the plasma region is typically higher than $1 \mathrm{kG}$, and nearly $1.2 \mathrm{kG}$ in the cathode dimple. A higher field allows for some field degradation due to the increased temperature in a running source. 

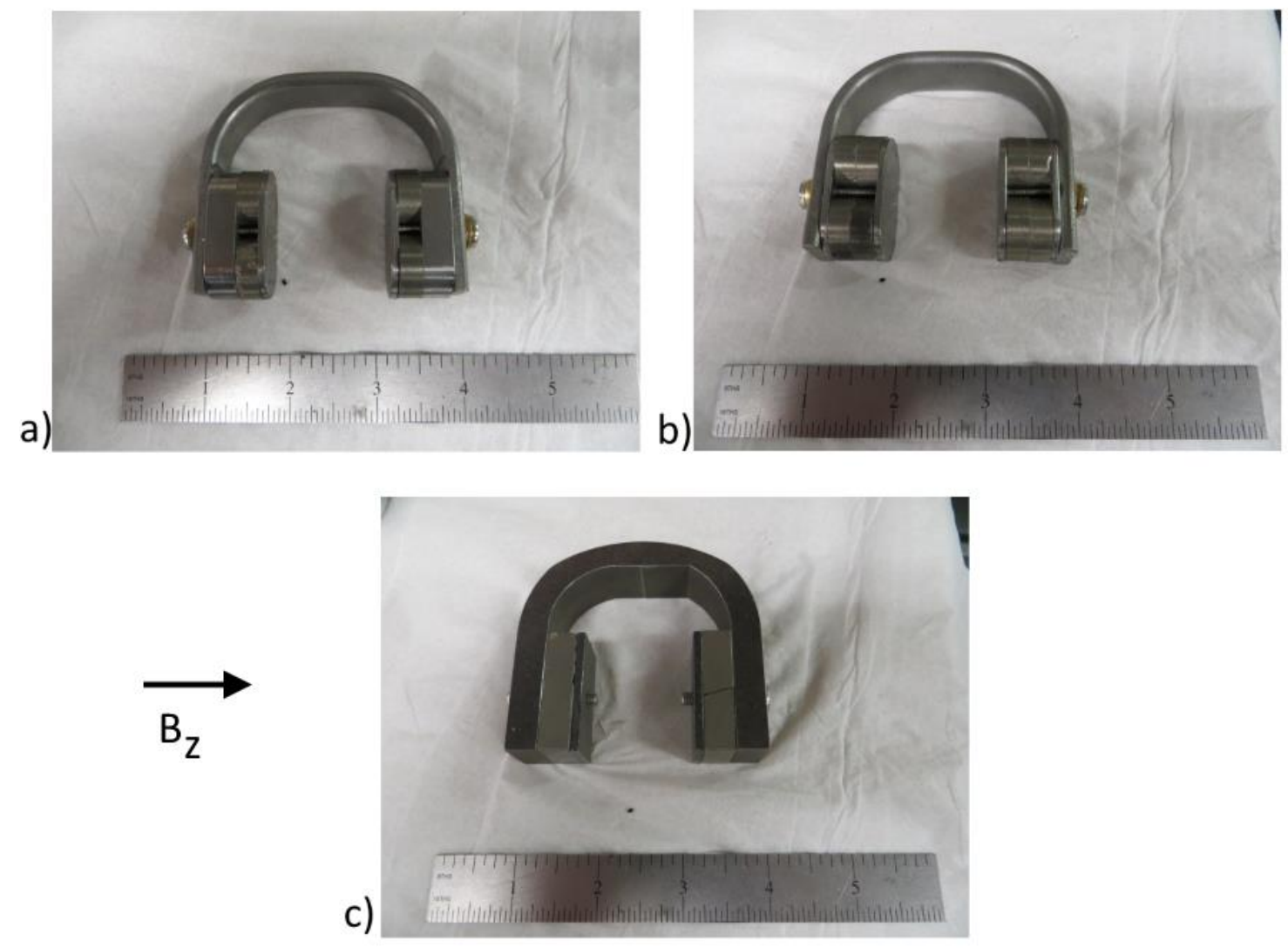

Figure 31: The RIL source magnetron permanent magnets and yoke. 4 magnets in (a), 8 magnets in (b), and 2 larger rectangular magnets in (c).

The magnetic fields were measured using a Hall probe mounted to a stand which had micrometer dials mounted to measure movement in all three axes. A scan of the $B_{z}$ field for each magnet was performed from the base of the magnet assembly up to 1.2 in above the base, well past the tip of the extractor cone as shown in Figure 32. The z-direction labeled here is not in the direction of beam travel. The same Hall probe has been used for all magnetic field measurements for these sources.

These permanent magnets also affected the roundness of the beam exiting the source. The $\mathrm{B}_{z}$ field is strong enough to create some dispersion as the $\mathrm{H}^{-}$beam passes the magnets. Original measurements on the LEBT emittance probes showed that the vertical emittance was routinely larger than the horizontal. As a test of this theory, the next source installation was rotated $90^{\circ}$ 
when mounted to the source cube. All emittance measurements from this installation had larger horizontal emittances. Currently operational sources are mounted such that the vertical emittance is larger than the horizontal.

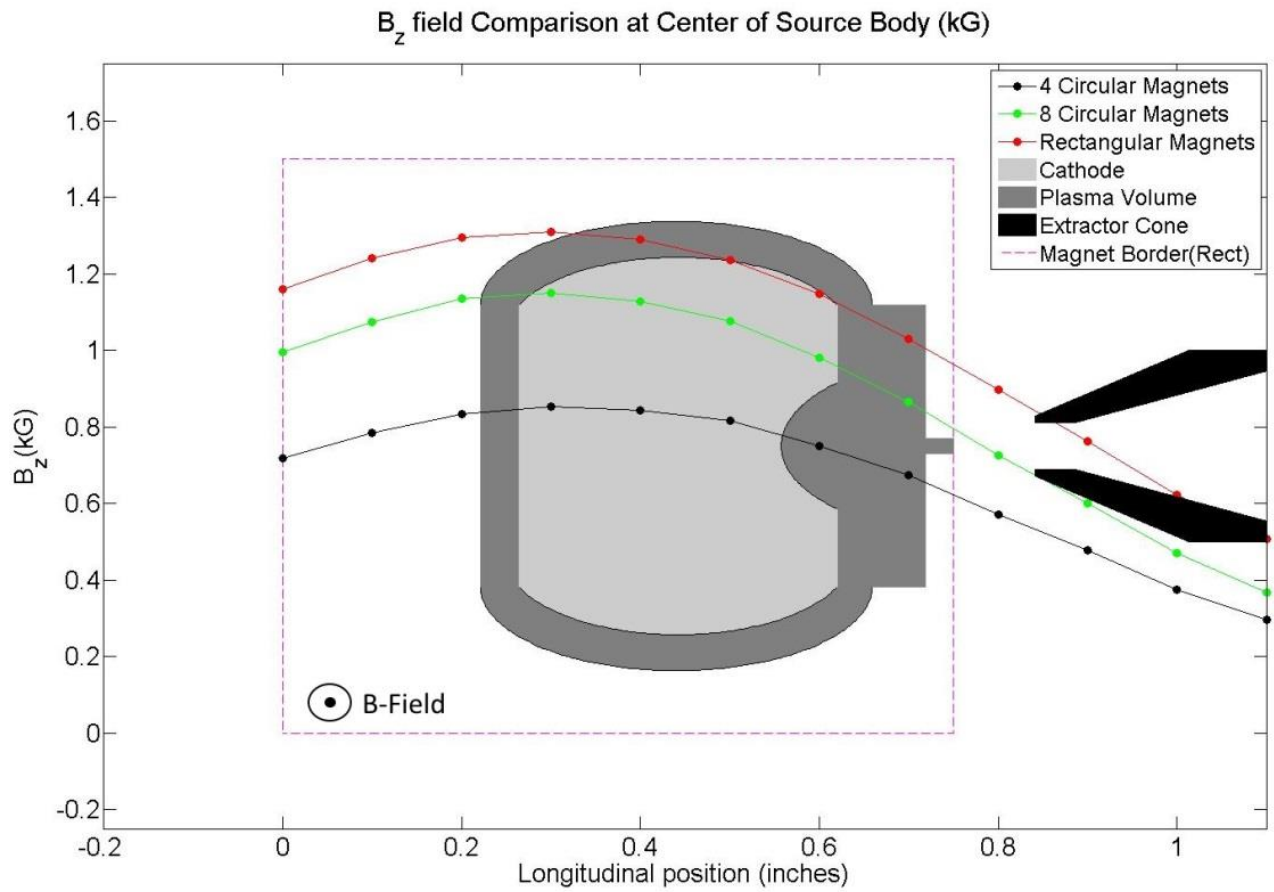

Figure 32: Plot showing relative $B_{z}$ field strength of the magnet designs overlaid with the source geometry. It is clear that the $B_{z}$ field strength in the plasma region is much improved with the rectangular magnets. $B_{z}$ direction is not the longitudinal direction of beam travel.

One possible concern with the permanent magnets was that the high source body temperatures would affect the magnetic field over time. If raised to a high enough temperature the samarium cobalt magnets would irreversibly lose some field strength. Studies were performed by heating a magnet assembly with 8 disc magnets on the original yoke to $120^{\circ} \mathrm{C}$ at atmospheric pressure using an industrial hot plate. The magnetic field was reduced at high temperature as shown in Figure 33a, but returned to normal upon cooling to room temperature. A thermocouple was attached to this magnet assembly while running a source in a test stand with the results shown in Figure 34. While the source arc was present over a period of 6 weeks 
the magnet temperature never exceeded $75^{\circ} \mathrm{C}$. The supplier lists the maximum working temperature at $300^{\circ} \mathrm{C}$, far out of the range of the source operation even when sources run hotter during start up. Lastly a magnetic field measurement is taken at the source plasma region and extraction gap each time a source is removed from operation and cleaned or rebuilt. The results are shown in Figure 33b from June 2013 until January 2015 and show no signs of long term loss of magnetic field strength.

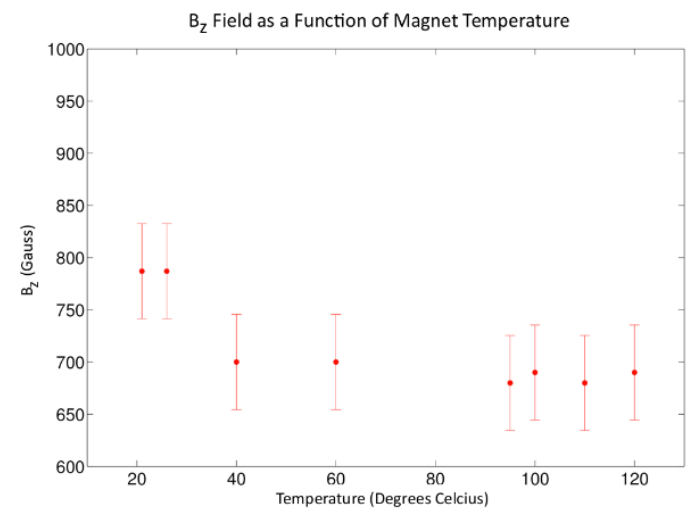

a)

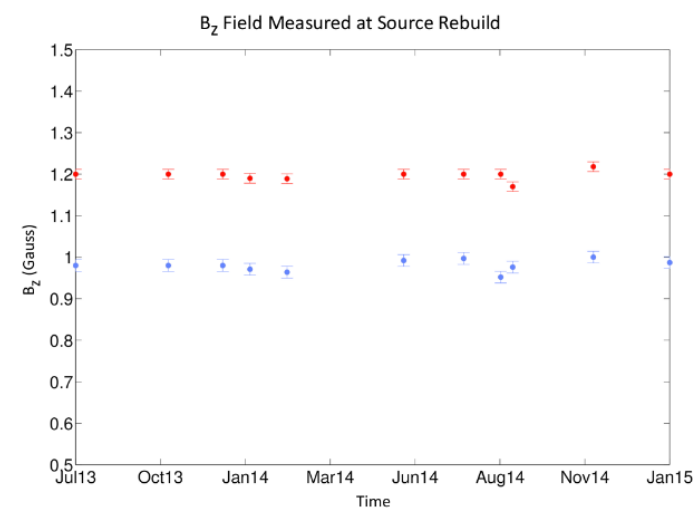

b)

Figure 33: Plots showing $B_{z}$ field vs temperature for the 8 disc shaped magnets design in (a) and over a period of almost 2 years of measurements for the rectangular magnet design in (b). This is the field measured in the plasma region above the dimple in the cathode (red) and in the extraction region (blue). 


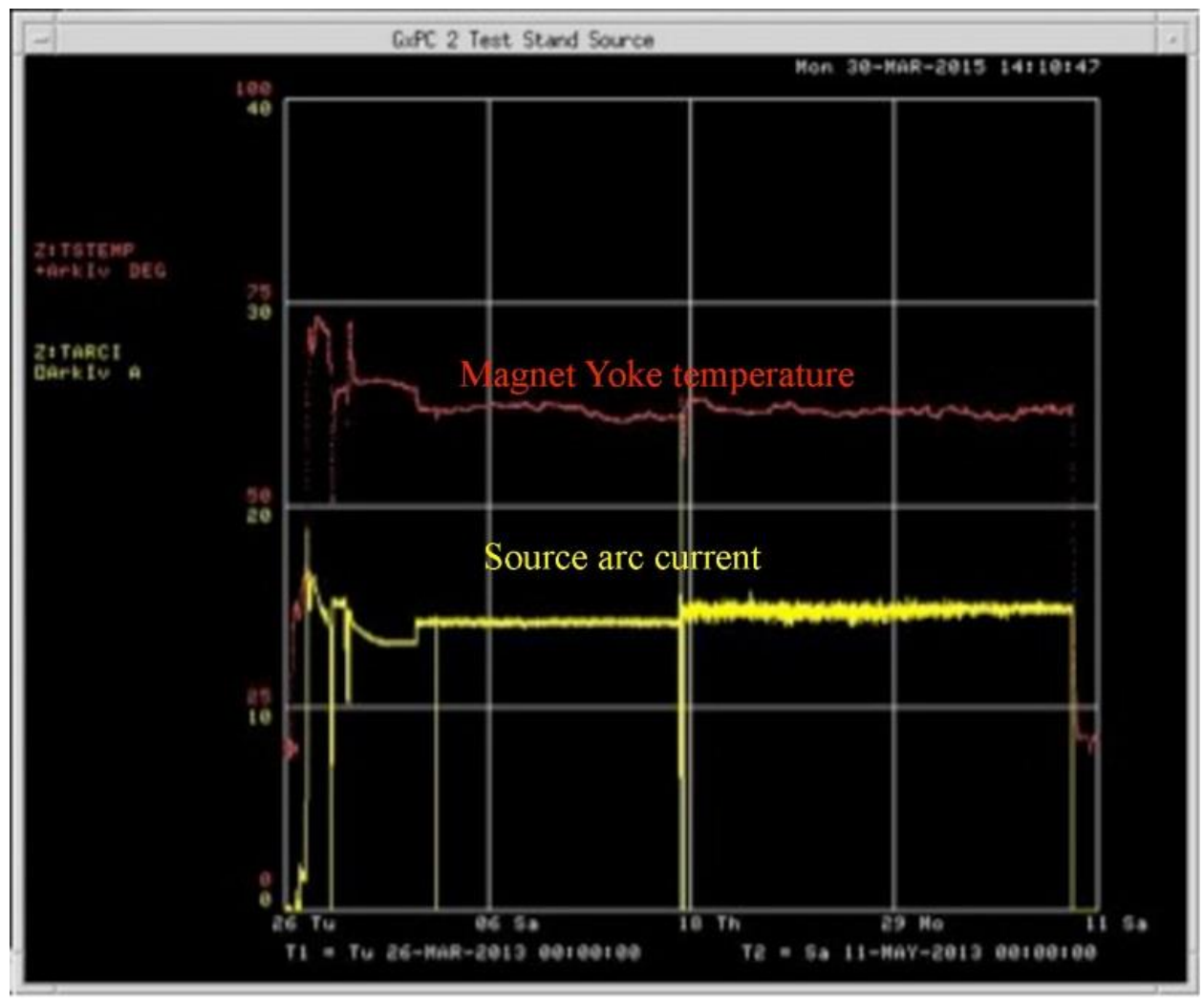

Figure 34: Plot showing magnet temperature during test stand source operation over six weeks. The magnets never get hotter than $75^{\circ} \mathrm{C}$.

\subsubsection{Source Vacuum Pressure}

The typical line of thinking when it comes to vacuum pressure and particle accelerators is that a lower vacuum pressure is always better for beam. As was seen in the LEBT with gas focusing and to a greater extent in the source cube this is not always the case. A magnetron source such as the one operated at FNAL has both upper and lower vacuum pressure boundaries.

The upper boundary is defined by the pressure limit at which $\mathrm{H}^{-}$ion stripping becomes evident. The extra electron in an $\mathrm{H}^{-}$ion is very weakly bound to the nucleus. At high enough vacuum pressures there are enough ions and electrons moving around with enough energy to be able to strip the $\mathrm{H}^{-}$ion of its extra electron. This results in a decreasing beam current output 
from the source when the vacuum pressure is raised. If the pressure remains this high for long enough, or is raised even higher, sparking in the source is more likely to occur. This is due to the free electrons creating sparks from the anode to the cathode. The mere presence of the extra gas from higher pressure is not likely to cause the source to spark from the anode to the cathode or from the anode to the extractor. It does however make it more likely to continue sparking once a spark does occur. Lowering the vacuum pressure in this instance will not only lessen the spark rate, but improve the beam output intensity as shown in Figure 35 . The extractor voltage plotted in red dives to zero during a spark as the voltage on the source goes to zero.

Wanting to avoid high source cube pressure, the original design for the cube vacuum system included two $1200 \mathrm{~L} / \mathrm{s}$ turbo pumps on the source cube. Keeping enough hydrogen flowing into the source to maintain stable plasma required the cube pressure to be between (1-2) $\times 10^{-6}$ Torr. Experience with sources run at this pressure level showed very high spark rates, sometimes with multiple sparks per minute.

The exact cause of the lower limit in the source cube pressure is unknown. There may be processes in the plasma that we are unable to measure. The geometry of the ion source makes it difficult to study the plasma directly. We are left with maintaining the ion source parameters in a regime that allows for steady ion source operation. One possible explanation for the excessive sparking lies in an understanding of the Paschen curve. 


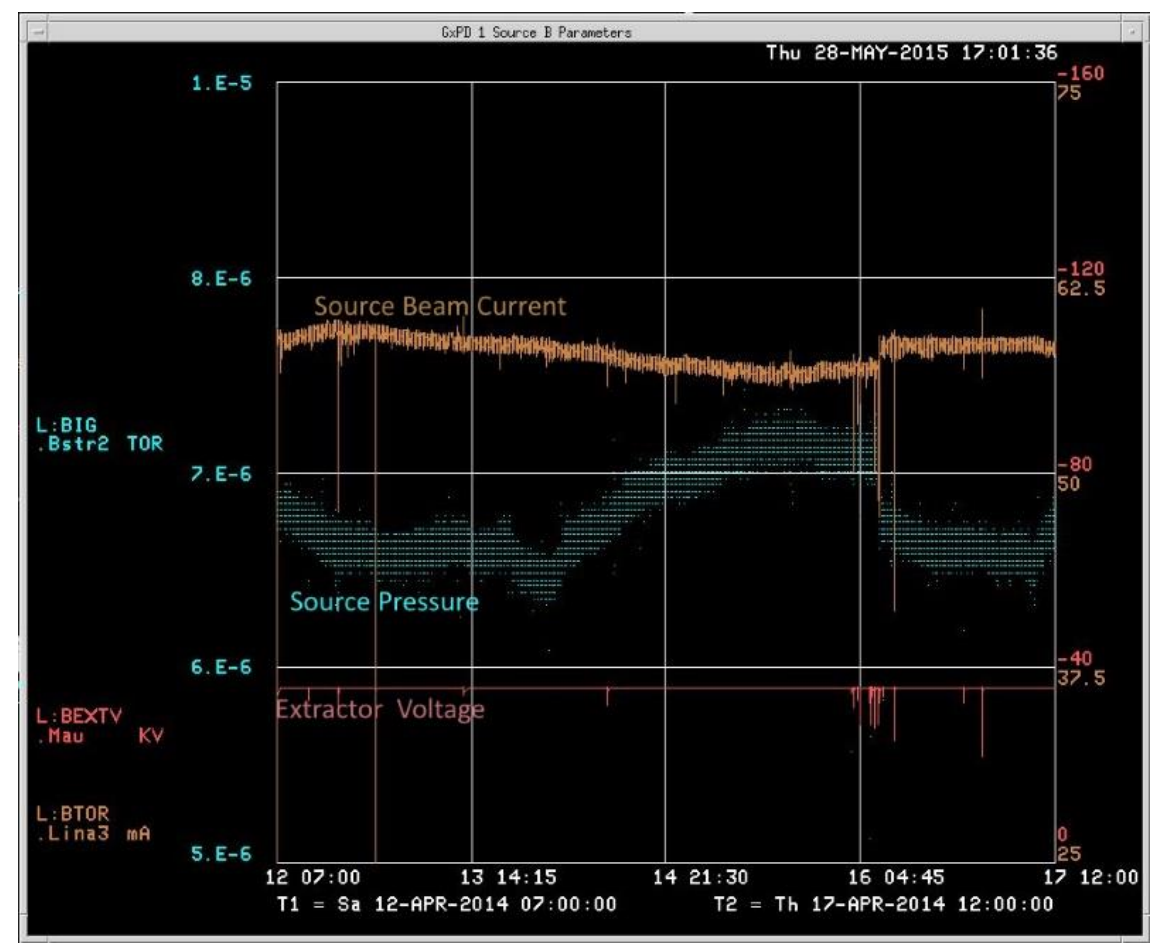

Figure 35: Plot showing the positive effect of lowering an already too high source pressure. Not only does sparking cease but beam intensity increases.

The Paschen curve relates the breakdown voltage of a gap to the pressure of a given gas between the electrodes. Equation 2, was discovered by F. Paschen in 1889 [10]

$$
V_{b r}=\frac{B \times(p d)}{\log \left(\frac{A \times(p d)}{\log \left(\frac{1}{\gamma+1}\right)}\right)}
$$

The breakdown voltage $V_{b r}$ is a function of the product of the electrode gap distance $d$, and the pressure of the gas $p$ between said electrodes. $A(\text { Torr } \times \mathrm{mm})^{-1}$ and $B(\mathrm{~V} / \mathrm{mm}) \times$ Torr $^{-1}$ are constants determined by the gas. $\gamma$ is a dimensionless constant determined by the metal of the electrodes related to the number of emitted electrons from its surface when impacted by a positive ion. For hydrogen, this curve is plotted in Figure 36. 


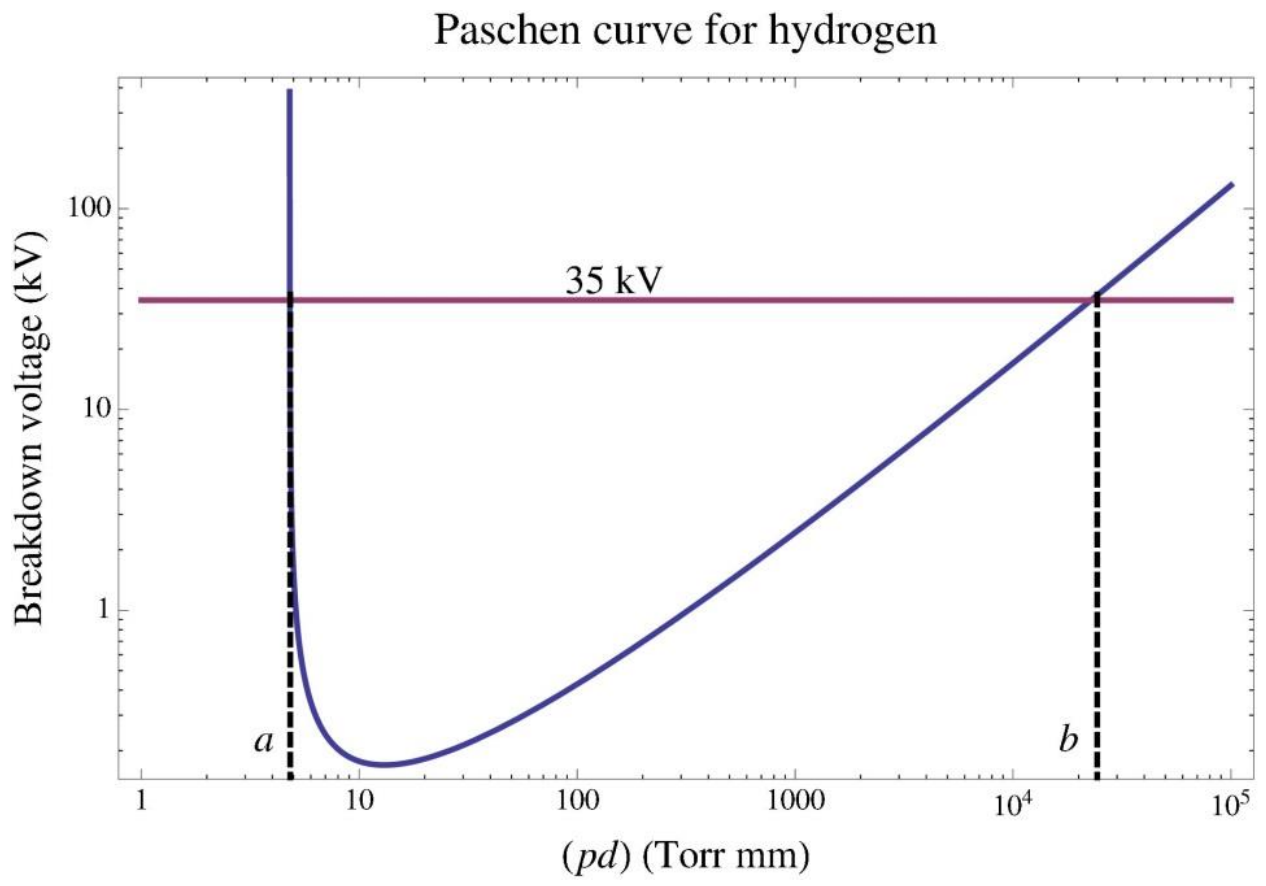

Figure 36: The Paschen curve for hydrogen gas. The voltage at which a spark is likely to occur is based on the product of the distance of the gap of the electrodes and the gas pressure between those electrodes.

An errant assumption early on led us to believing that the source would operate in the part of the graph to the left of the breakdown voltage minimum, safe from sparking. This mistake was regarding the vacuum pressure as read from an ion gauge on the source cube as the actual vacuum pressure between the electrodes. The electrodes in this instance are the extraction cone tip and the anode cover plate. The distance between these two is tightly controlled when assembling the source and is set to (0.90-0.95) inches. The pressure as read from the ion gauge was $2 \times 10^{-6}$ Torr. The problem is that this is an average pressure reading from the ion gauge for the entire beam cycle of $66 \mathrm{~ms}$. This ion gauge is not close to the electrode gap, nor does it read back the instantaneous pressure when the hydrogen gas is injected into the ion source. The instantaneous pressure in this gap can reach 10's of Torr. This meant that the ion source was operating on the right hand portion of the curve in Figure 36 and that lowering the source vacuum pressure as was standard procedure would actually increase sparking, not reduce it. 
Without a way to measure this instantaneous vacuum but certain that it was much closer to 1 than $1 \times 10^{-6}$ Torr, an attempt to run a source with a much higher average pressure was performed. This was done by turning off one of the two $1200 \mathrm{~L} / \mathrm{s}$ turbo pumps on the source cube resulting in a vacuum pressure of $8 \times 10^{-6}$ Torr. Experience with average vacuum levels much above this had shown $\mathrm{H}^{-}$ion stripping and sparking as mentioned above. This large shift in pressure should move the product $p d$ to the right on the Paschen curve thereby increasing the breakdown voltage. The results of this change are shown in Figure 37. While the spark rate did not change immediately, it did result in a greatly reduced spark rate over time. Further experimenting with average cube vacuum pressure showed a lower limit of $5 \times 10^{-6}$ Torr before sparking again became common. This combined with the upper limit derived from $\mathrm{H}^{-}$ion stripping gives an operational range of $(5-9) \times 10^{-6}$ Torr for the average vacuum.

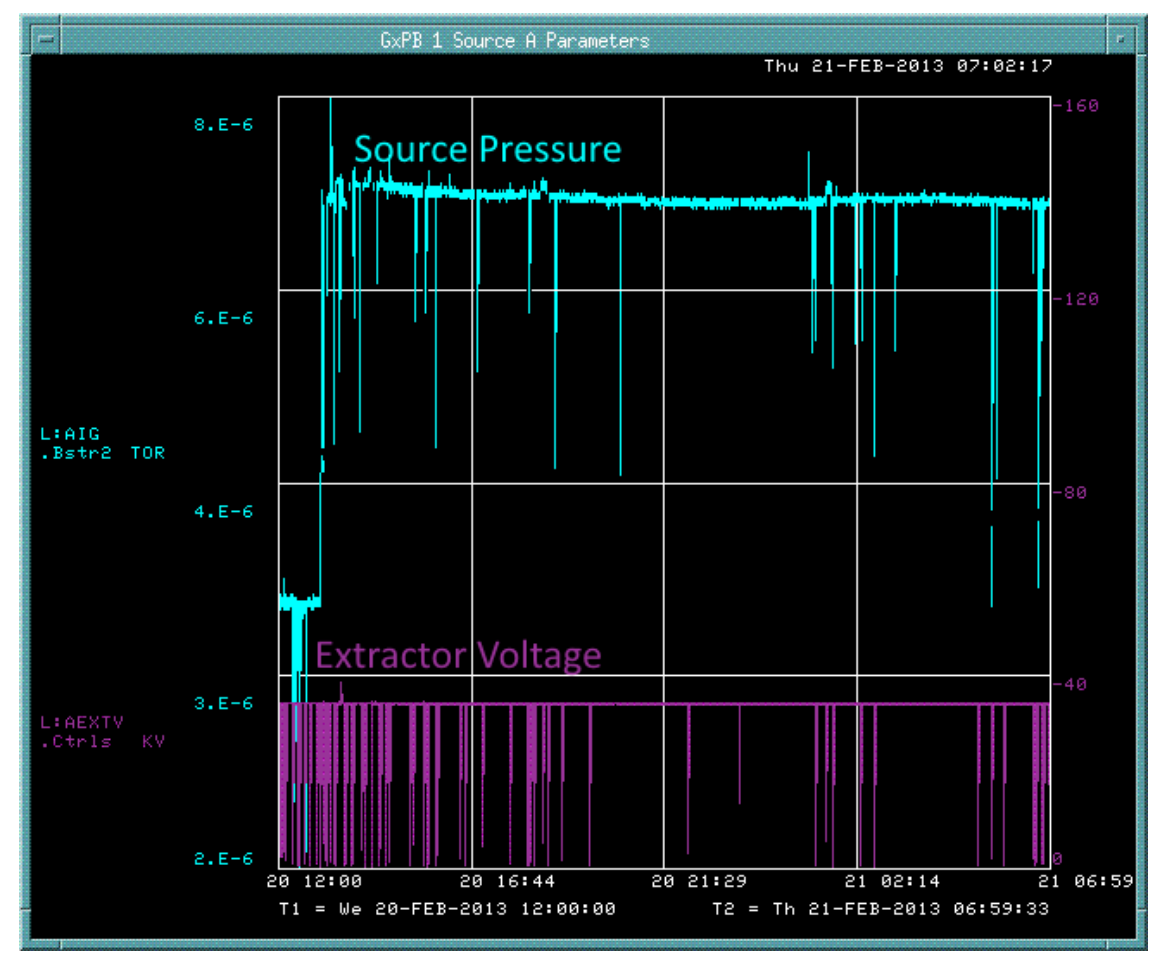

Figure 37: Plot showing the effect of increasing the source pressure away from the minimum of the Paschen curve. Extractor sparking seen in the purple trace's drops to $0 \mathrm{kV}$ reduces within hours. 
An added advantage of this change was that only one of the source turbo pumps was required for operation. The bottom turbo pump for the source cube was removed and the space was used to mount the hydrogen bottles underneath the slide on which the sources and LEBTs are mounted. This means that when a source change is required the gas line is no longer broken interrupting gas flow to the source. As the turbo pumps are able to withstand the motion of the slide when changing sources, the change can be done with both sources still operating. The extractors are turned off for safety, but the arc is still pulsing within the cube. This allows for a source change to be completed in less than an hour with no source recovery from contamination.

Even without discovering the distinct cause of the low pressure limit for the ion source operations, it has been empirically shown that raising the vacuum pressure above $5 \times 10^{-6}$ Torr does lead to lower spark rates in the extraction region. This alone is not enough to stop the sparking. Another important factor is maintaining proper control of the cesium layer on the cathode surface.

\subsubsection{Cesium Layer on Cathode}

A magnetron source can maintain plasma without cesium, however the arc discharge is quite low at only 2-3 A which provides less than $10 \mathrm{~mA}$ beam output from the source. In studies at Novosibirsk, V. Dudnikov and his colleagues discovered that introducing a cesium vapor into the magnetron source increased the $\mathrm{H}^{-}$production significantly [11]. At FNAL, introducing cesium to the magnetron resulted in a beam output of $70 \mathrm{~mA}$ from the old magnetron design. The cesium vapor adhered to the molybdenum cathode surface lowering the work function. 
The molybdenum cathode has a work function of $4.36 \mathrm{eV}$ while cesium has a work function of $2.14 \mathrm{eV}$. A suitably thin layer of cesium deposited on the molybdenum surface has an even lower work function closer to $1.5 \mathrm{eV}$ [9]. More electrons are freed from the surface and are able to combine with the hydrogen atoms to create a higher yield of $\mathrm{H}^{-}$ions. This optimal cesium layer is difficult to maintain.

In the previous versions of the FNAL magnetron sources, the arc discharge current was kept as high as $50 \mathrm{~A}$. The temperatures of the source body and cathode were kept at $300{ }^{\circ} \mathrm{C}$ and 430 ${ }^{\circ} \mathrm{C}$ respectively. In order to replenish cesium that will be thermally ejected from the cathode surface, these magnetron sources require a constant flow of cesium. The cesium boiler was kept at or above $140{ }^{\circ} \mathrm{C}$ to provide enough cesium to operate.

With the new magnetron design, the arc discharge current is kept at $15 \mathrm{~A}$ which results in typical source body temperatures between $160{ }^{\circ} \mathrm{C}$ and $200{ }^{\circ} \mathrm{C}$ and cathode temperatures between $350{ }^{\circ} \mathrm{C}$ and $380^{\circ} \mathrm{C}$. These lower temperatures will boil off the cesium layer more slowly which means that the cesium boiler temperature can be lowered to reduce the cesium flow.

While the cesium flow is too low to be measured as it is in the $\mathrm{nm} / \mathrm{s}$ range, it can still be controlled and observed in the source operation. It was noticed during times when the cesium boiler heater power supply would trip and no longer boil cesium. The source spark rate would decrease following these trips. Deducing that the cesium layer on the source was too thick, the boiler heater power supply current was lowered in stages, typically $3-6^{\circ} \mathrm{C}$ at a time. As can be seen in Figure 38 this did lead to lower source spark rates, eventually. There is a substantial lead time of (16-24) hours between when the change is made and when it seems to affect the spark rate. However with no other change made, it has been seen repeatedly that a change in the cesium boiler temperature on one day will affect the source spark rate on the following day. 


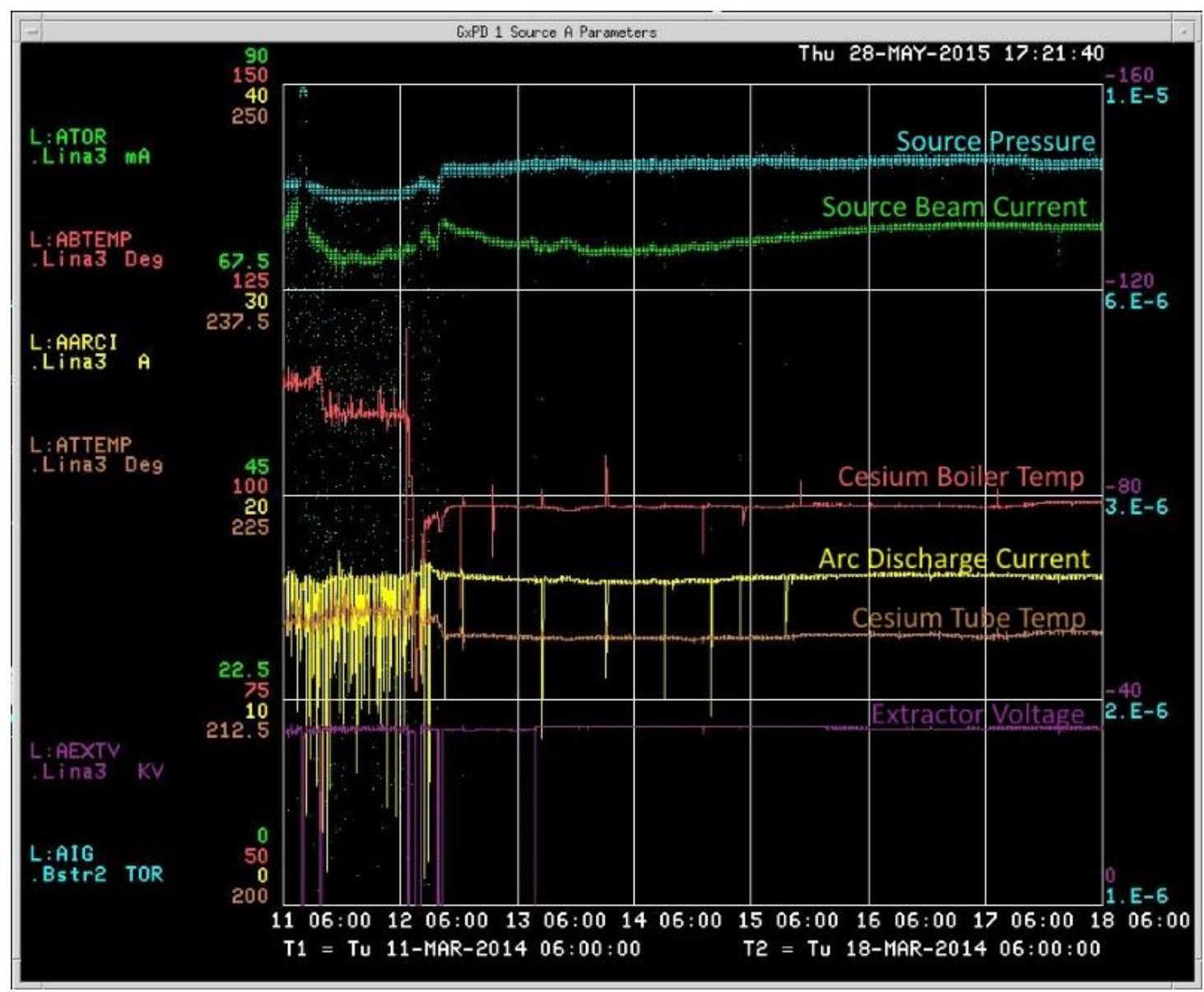

Figure 38: Plot showing that the decrease of the cesium boiler temperature, and thus cesium flow into the source leads to reduced sparking after 16-24 hours. Early attempts at source operation copied heater temperatures from the C-W sources that had to be reduced for RIL sources.

The boiler is only one part of the cesium delivery path to the source. There is also a valve to isolate the boiler from the source and a tube from the valve to the source body that measures roughly 11 inches long. These parts are heated with separate power supplies. To investigate the cesium level and its effect on sparking, changes can be made to the tube and valve to change the flow through them with a shorter time constant than the 16-24 hours of the boiler changes. For optimal source operation, a delicate balance between these temperatures must be found where each element closer to the source is warmer than the previous one.

An operational source can run for a day or two without a spark with the cesium system under control. Compared to early spark rates of several sparks per minute, the new design and 
operation of the magnetron sources have come a long way. With a stable running source, it was possible to spend time trying to advance the design for further improvements in intensity, efficiency, and quality.

\subsection{Further Magnetron Source Research}

The arc discharge current is the primary indicator of the health of the source. Most efforts to tune a source for output or efficiency revolve around this current being stable at a nominal value. Once a source is running stably it is typically not adjusted until the arc discharge current starts to vary enough to affect the beam output. Thus it is important to remove dependencies that vary over time or due to ambient temperature. It is also important to be sure to maintain a plentiful plasma volume to ensure the arc discharge is held high during the entire beam pulse.

\subsubsection{Cathode Geometry and Plasma Volume}

The FNAL magnetron source requires a long arc pulse to allow for enough time for the extractor pulser to rise to $35 \mathrm{kV}$ which is approximately $110 \mu \mathrm{s}$. Once the extractor has reached $35 \mathrm{kV}$ the $\mathrm{H}^{-}$beam traveling through the LEBT requires another $60 \mu$ s for ion neutralization. This leaves the last $60 \mu$ s of the $230 \mu$ s beam pulse for useable beam to any user. In order to sustain a flat arc discharge through this pulse length the plasma must not become rarified, or lacking in hydrogen. One recommendation to prevent rarefied plasma is to increase the plasma volume so that the arc has more plasma from which to extract the $\mathrm{H}^{-}$ions.

Alessi and Sluyters from BNL [12] showed that increasing the space between the anode and the rear of the cathode produced a stable arc discharge at a lower pressure point than the normal grooved magnetron with an increased emission current density. It was suggested by 
Wiesemann [13] that a magnetron with an asymmetric gap to the back of the cathode will have a larger plasma volume that will allow the plasma to spread around to the extraction region due to the ExB drift of the plasma. The increase of the total plasma volume could lead to a more stable arc discharge with the higher emission current density leading to less noise on the produced $\mathrm{H}^{-}$beam.

The old magnetron cathodes eventually extended the groove around the entire cathode body, not just the side facing the extraction slit. The new style magnetron has just a dimple facing the extraction aperture. A series of cathode designs were tested [14] that varied the cathode shape and decreased the cathode volume, thus increasing the plasma volume. These variations to the cathode shape are shown in Figure 39. Baseline comparisons were taken with a normal dimpled cathode as seen in (1). A shallow groove was cut on the back of that cathode (2) which then led to a deeper groove on the back (3). This groove was then extended allaround the cathode body to the edges of the dimple (4). These were all performed on the same cathode 'blank' in order to save materials and machine shop time.

V. Dudnikov suggested another method of increasing the plasma volume which was then attempted in the test stand [15]. The new design labeled a hollow cathode is a cathode body with a cylinder cored from the cathode body facing the cesium inlet. This method has been attempted in other surface plasma sources. A $3 \mathrm{~mm}$ diameter hole was drilled $6 \mathrm{~mm}$ deep into the side of the cathode as seen in Figure 39 (5). The hole was supposed to be placed opposite the cesium inlet to attain the best results as the ExB drift would pull $\mathrm{H}^{-}$ions from the hole towards the front of the cathode. The cathode connection to the arc power supply is also on this side preventing the hole from being placed on this side. 


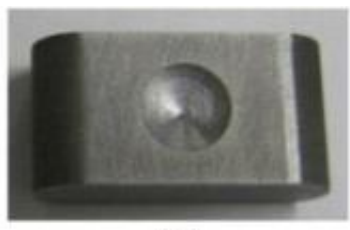

1a)

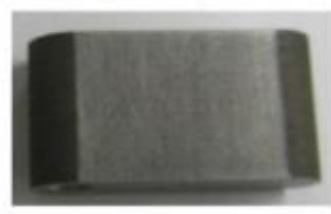

1b)

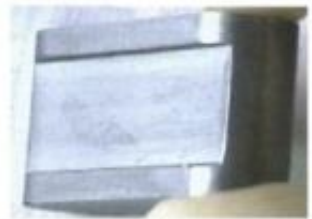

2)

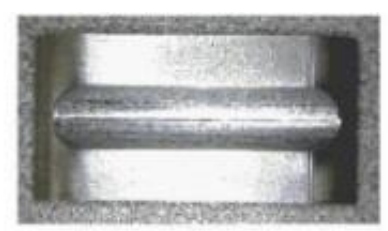

3)

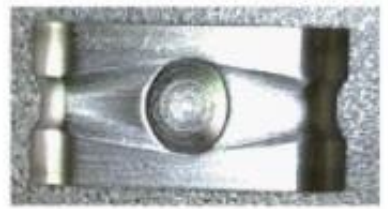

4a)

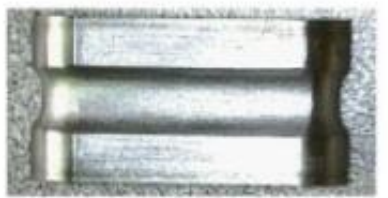

4b)

Figure 39: Cathode surface evolution for tests: (1) normal dimpled source, (2) shallow grooved back, (3) deep grooved back, (4) all-around grooved, (5) hollow cathode.

After testing the hollow cathode with the hole on the far side of the cathode another attempt was made with the magnetic field reversed for the source thus creating the proper direction for the ExB drift. Further pursuance of this design would require a change in the mounting of the extractor because the reversed field would bend the co-extracted electrons from the source directly toward one of the mounting posts creating a possible spark path.

Once each source was holding a steady arc discharge, emittance measurements, as well as, beam noise calculations were performed to look for any changes in beam quality. The beam current was measured with both a toroid and a Faraday cup to determine if the increased plasma volume was creating a less noisy beam. These signals, as well as, the extractor voltage were measured on a $500 \mathrm{MHz}$ oscilloscope. The RMS and mean values were measured over a $70 \mu$ s portion of the extracted beam flattop that is typical of the operational sources. The ratio of the RMS beam current to the mean value of the beam current for each cathode shape is plotted in Figure 40a. The pressure reported is the average pressure of the source cube with the 
ion source can sitting inside the cube. There is a $1000 \mathrm{~L} / \mathrm{s}$ turbo pump providing the vacuum pumping for the source. This cube extracts to the test stand which is a large steel vessel of approximately $18 \mathrm{ft}^{3}$ that has another $2200 \mathrm{~L} / \mathrm{s}$ turbo pump on it.

The shallow grooved source performs quite similar to the standard dimpled cathode albeit with higher noise than all the other cathodes. The cathode grooved all-around performs similarly to the reversed B-field hollow cathode, but not as well as the hollow cathode with the normal B-field. The deep grooved cathode created the least noisy beam especially at high pressures. Unfortunately operational sources cannot operate at pressures above $2 \times 10^{-6}$ Torr. At low pressures, approaching operational cube values, the normal cathode and the hollow cathode with the reversed B-field exhibit the least noise on the beam. Unfortunately the test stand sources cannot maintain a stable arc at this low pressure.

Figure $40 \mathrm{~b}$ shows the source output beam current as a function of the gas pressure and gives a good indication of when $\mathrm{H}^{-}$stripping becomes evident in the source. These tests were done with the extractor voltage at $28 \mathrm{kV}$ because extractor sparking is prevalent at high pressures at $35 \mathrm{kV}$ in the test stand. For all the various cathodes, $\mathrm{H}^{-}$stripping becomes apparent above $1.6 \times 10^{-6}$ Torr. The shallow grooved cathode created the highest beam output at slightly higher pressure than the normal dimpled cathode. The all-around the cathode grooved source preformed the worst, with at least $5 \mathrm{~mA}$ lower beam current than even the normal dimpled source. The deep grooved cathode performs better at higher pressures as it did in the beam noise tests. Its maximum output is still almost $10 \%$ below that of the normal dimpled cathode. Again this pressure is far too high to operate a source in the RIL. 


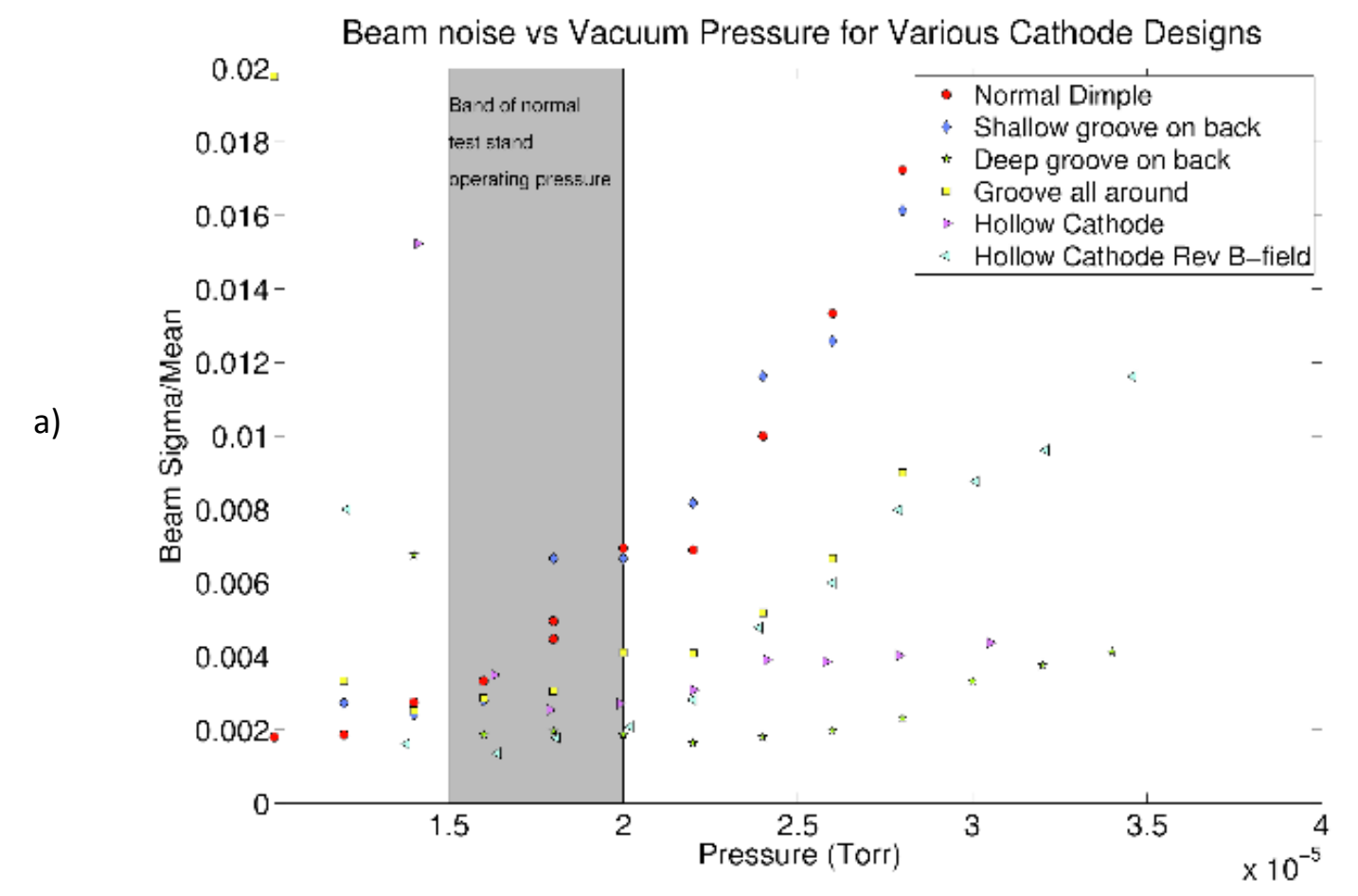

Beam Current vs Vacuum Pressure for Cathodes at $28 \mathrm{kV}$ Extraction Voltage

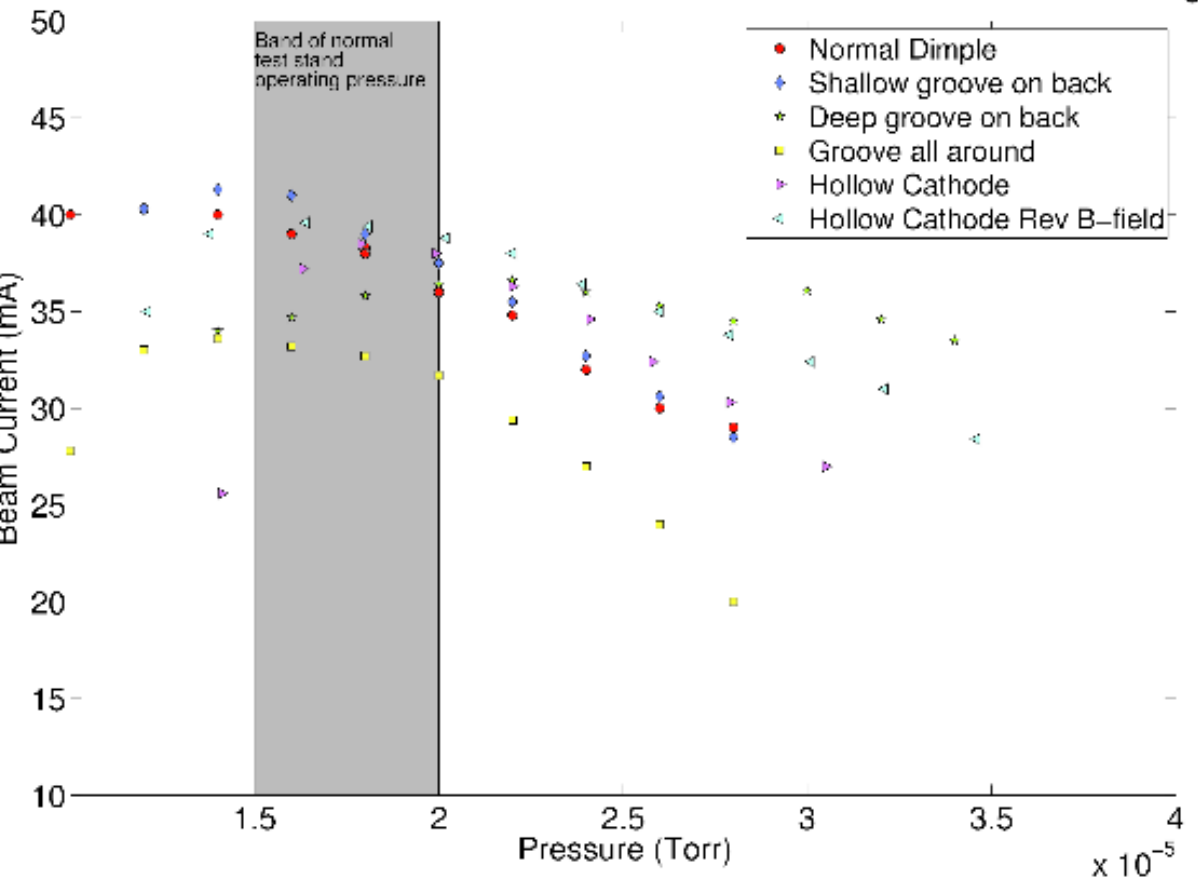

Figure 40: Plots comparing experimental cathode surfaces. Beam noise versus pressure is shown in (a) and beam output current versus pressure is shown in (b).

Emittance numbers for the various cathodes are compared in Figure 41. The vertical emittances are always larger due to the permanent magnets on the source. The horizontal 
emittances of the deep grooved cathode and the all-around grooved cathode are the smallest but that could also be due to the lower intensity. The shallow grooved emittances are smallest vertically but the second largest horizontally with the highest beam intensity. The hollow cathode, even with a reversed B-field, was able to maintain beam intensity without overly increasing the emittances. The vacuum pressure issues with these measurements due to the limitations of the test stand will be discussed in Section 5.4.5.

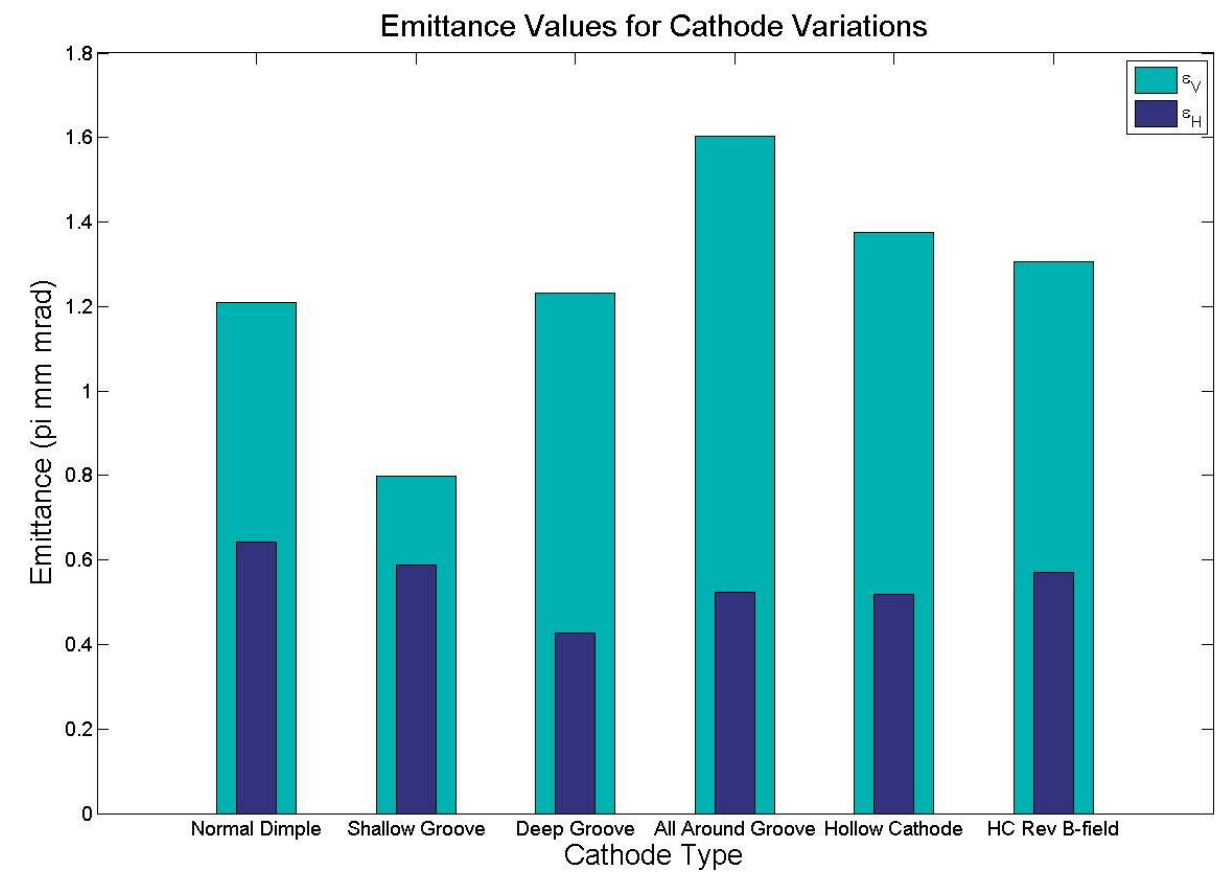

Figure 41: Comparison of emittances measured with each cathode surface.

\subsubsection{Nitrogen Doping of Hydrogen}

Another method found while researching methods to stabilize arc discharge current and decrease beam noise was the introduction of nitrogen to the hydrogen gas used to fuel the plasma. In the early 1980's tests were done at LANL on [16]. These tests show that even though the mechanism is not understood, that a small percentage of nitrogen gas added to the 
hydrogen gas has a quieting effect on the arc discharge current. Tests with hydrogen gas doped with small percentages of nitrogen were performed in the same manner as the cathode studies. Figure 42 shows the beam noise and extracted beam current results. The best results were found with $1 \%$ nitrogen at low pressures while with $3 \%$ nitrogen, it was difficult to keep a stable arc. An emittance comparison is shown in Figure 43. The $1 \%$ nitrogen mixture beam size is closest to the pure hydrogen gas while the $0.25 \%$ and $0.5 \%$ nitrogen tests show an increasing disparity in the emittances.

A potential problem with using nitrogen is that the vendor could not guarantee the required percentage. The bottles listed a variance of $+/-5 \%$. While differences were found between the $0.25 \%, 1 \%$, and $3 \%$ there is no way at present to verify the percentage listed on the bottle or to ensure that successive bottles would have the same percentage. Unfortunately as with the cathode geometry tests, conclusive results of these tests are hard due to the test stand limitations. 

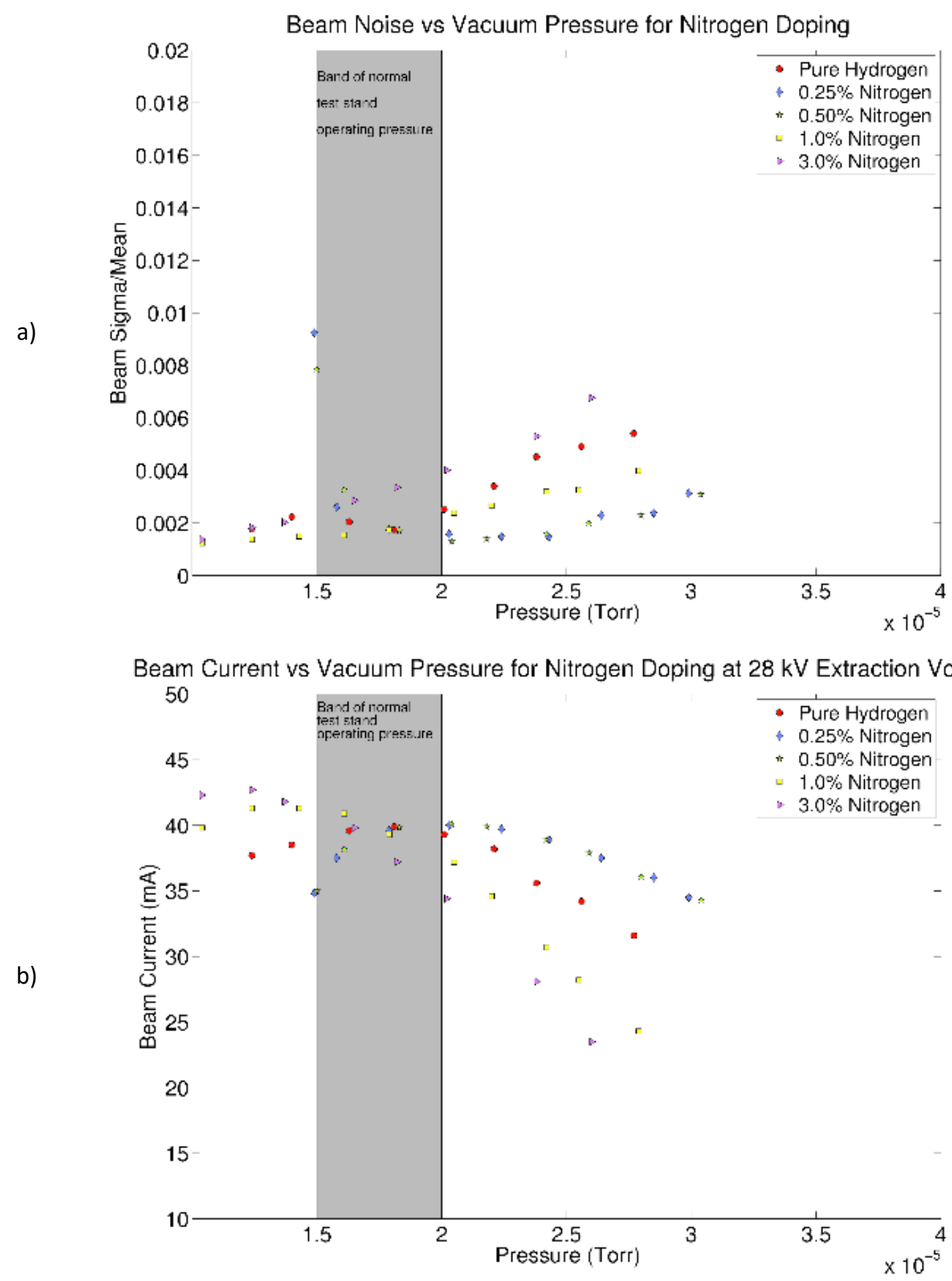

Figure 42: Plots comparing levels of nitrogen doping in the hydrogen gas. Beam noise versus pressure is shown in (a) and beam output current versus pressure is shown in (b). 


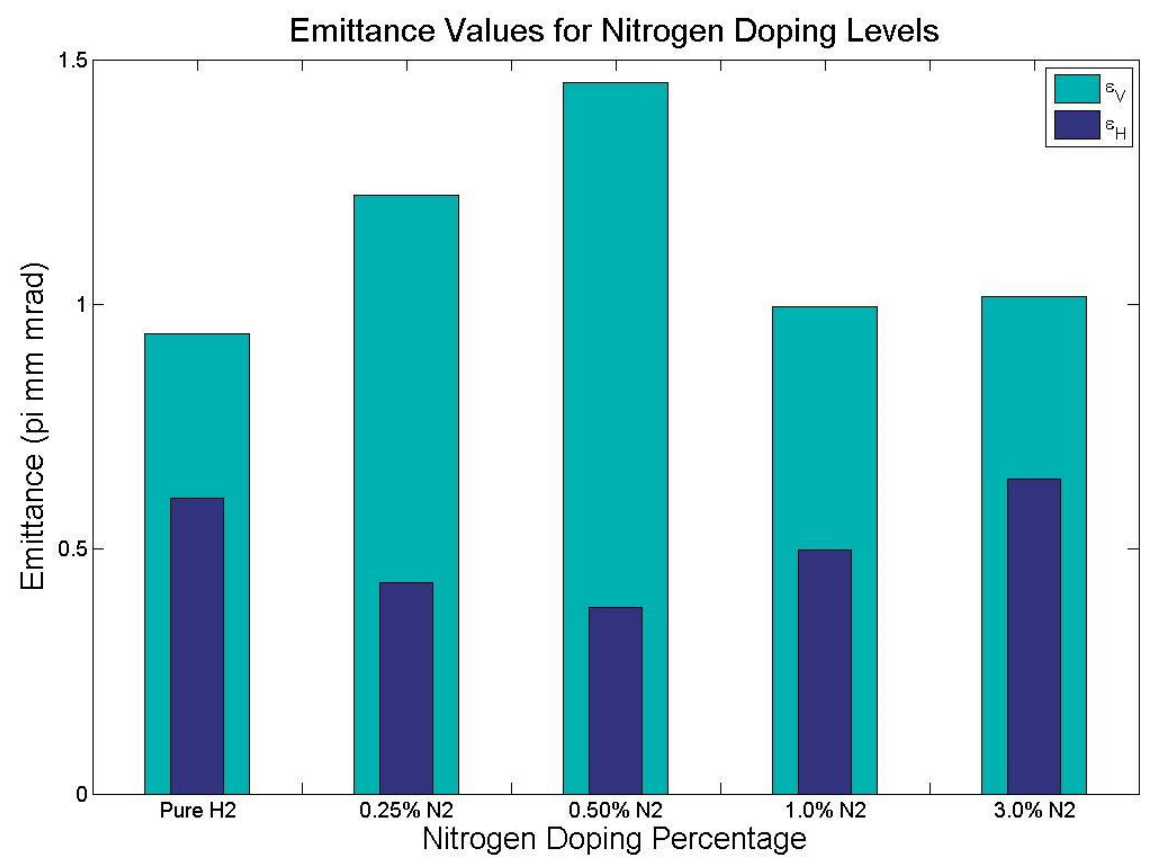

Figure 43: Comparison of emittances measured with each level of nitrogen doping in the hydrogen gas.

\subsubsection{Limitations of Current Test Stand}

The cube that the source sits in has a volume of $1000 \mathrm{in}^{3}$. This volume is reduced by the source can sitting re-entrant in the cube, leaving a volume of roughly $800 \mathrm{in}^{3}$ that is required to be kept under vacuum during operation. It is this volume where the source ion gauge sits and reports back the average vacuum pressure of the source. The source cube opens up to the LEBT through a 1 in diameter hole. The LEBT beam pipe through to the RFQ has a volume of $584 \mathrm{in}^{3}$ as it is 46.55 in long by 4 in diameter. The LEBT has two $350 \mathrm{~L} / \mathrm{s}$ turbo pumps that provide the vacuum.

The test stand where these sources were run for these tests contains a much larger vacuum volume of $18 \mathrm{ft}^{3}\left(31,104 \mathrm{in}^{3}\right)$ shown in Figure 44. Some test equipment such as a Faraday cup and emittance probes sit within this space, but the majority of this space is open and requires pumping to be kept at vacuum pressures when the source is operating. With the vacuum 
equipment available it is typical to maintain a pressure of $(1.5-2.0) \times 10^{-5}$ Torr with a source in operation. This is higher than the operational sources can reliably run. This difference alone makes the larger part of the data set for the emittances hard to translate to the operational sources. As a result of the increased vacuum and close proximity of the diagnostic equipment in this test stand to the source extractor it is unable to operate for extended times at $35 \mathrm{kV}$ without sparking. Experience with this test stand has played a part in defining the upper limit of the vacuum pressure in the operational sources.

A new test stand is under construction, and once completed will allow for these tests to be redone with similar settings to the operational sources and beam line. It will be a mockup of the operational cube and LEBT with the ability to attach emittance probes, multiwire cans, and other diagnostics.
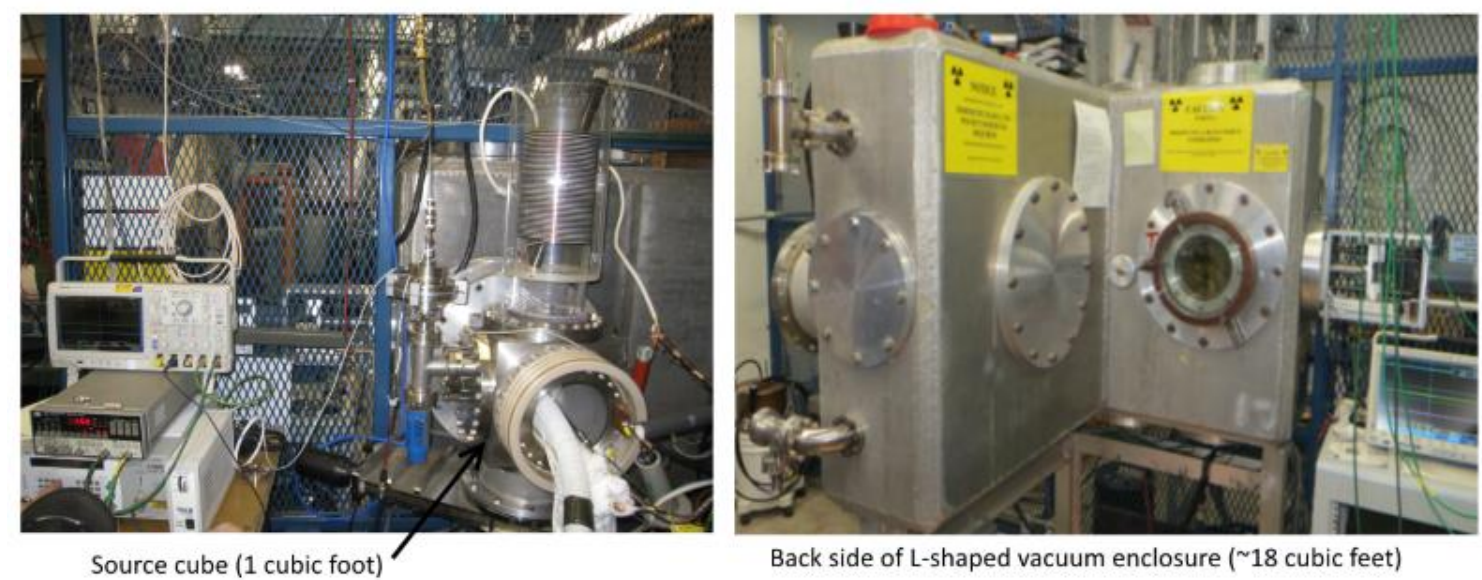

Back side of L-shaped vacuum enclosure ( $\sim 18$ cubic feet)

Figure 44: The test stand vacuum enclosure showing attached source cube (a) and opposite side with numerous flanges and ports (b).

\subsubsection{Solenoid Gas Valves}

It is important to maintain tight control over the source gas pressure, because it is one of the major parameters used to control the arc discharge current as well as the beam current. 
The valve used to control the gas injected into the source is a piezoelectric valve that has been in use for decades. Applying a voltage of approximately $100 \mathrm{~V}$ causes the piezoelectric crystal to flex allowing the hydrogen gas into the source. These valves typically require a negative offset voltage to force them closed to prevent gas leaking through the valve when it does not close properly. The piezo electric crystal is also very temperature sensitive. Figure 45 shows the effect of ambient temperature variations on the valve as reflected in the source gas pressure.

When the ambient temperature increases, the valve becomes harder to flex and allows less gas through the inlet, thus reducing the source gas pressure. Once the ambient temperature decreases, the valve becomes more flexible and allows the hydrogen gas to seep past the crystal into the source, thus raising the pressure.

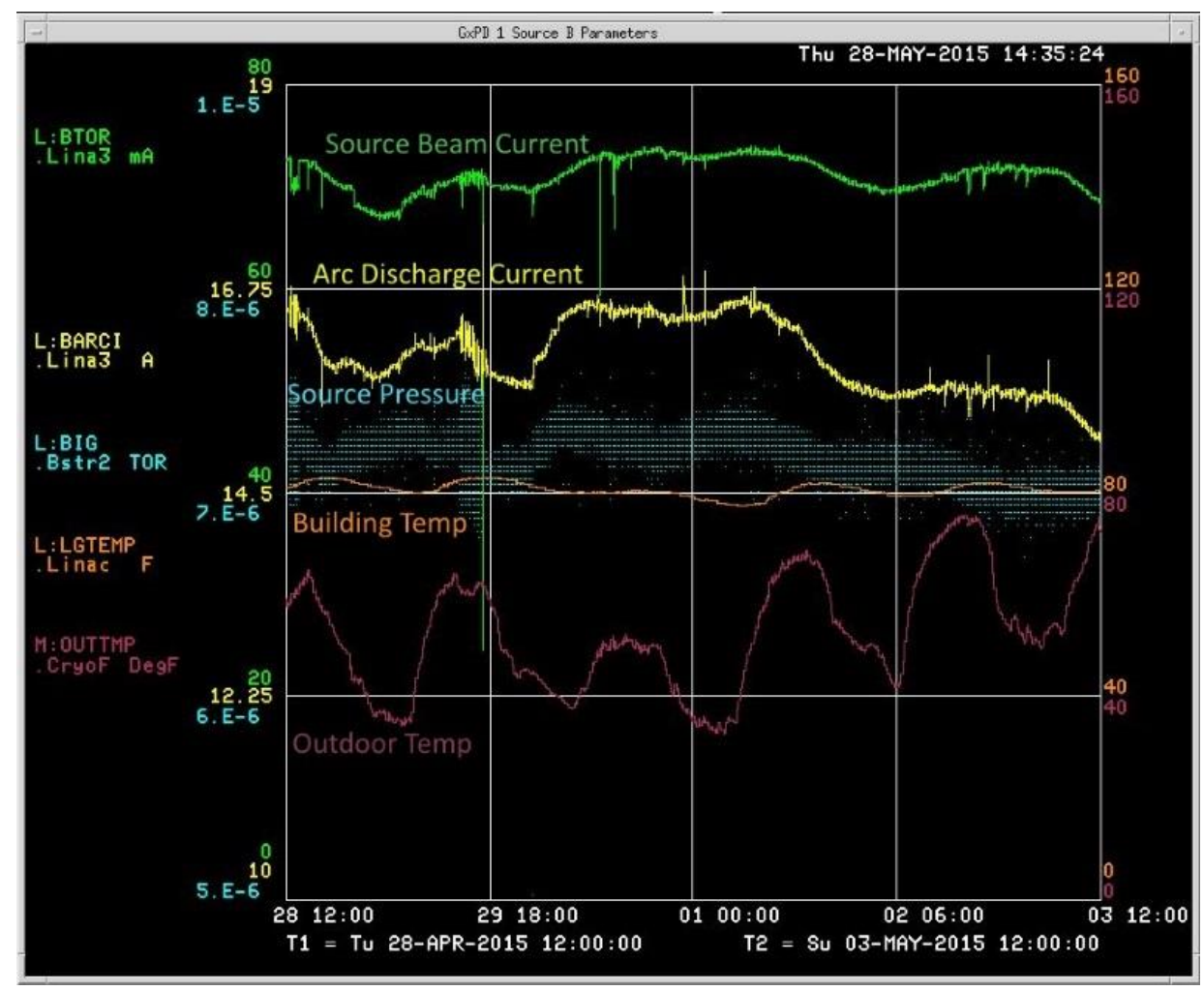

Figure 45: Plot showing the effect that temperature can have on the ion sources. The room containing the sources is open on two sides to the larger Linac gallery so it is impossible to control the temperature. 
The attempts made to regulate the gas pressure with a control loop that was designed for the old sources gave little benefit. The cooling system in the old Cockcroft-Walton domes did a better job of moderating temperature swings than the new source area. The new sources do sit inside the same enclosure as the old sources. There are now large openings in two of the walls of the room. These holes make it next to impossible to shield the sources from ambient temperature swings. It would be difficult to cool the high voltage enclosure around the source connections with the cesium system heaters which are also present in the enclosure. This impasse to cooling has led to searching for a new style of valve that would not be as susceptible to these temperature swings. A solenoid valve was procured from Parker Hannifin Corporation and is being tested to ensure it is a suitable replacement.

A test valve was installed on an operational source once it was verified to be able to produce gas bursts similar to the piezoelectric valve. This source ran for a month with the new valve. A failure mode was identified during temperature dependence tests. The solenoid valve uses a small PTFE polymer poppet to seal the aperture when the valve is closed, see Figure 46. When the valve body reached $54{ }^{\circ} \mathrm{C}$ the poppet melted. The valve runs closer to $30{ }^{\circ} \mathrm{C}$ so this is not a problem under normal conditions.

When the valve was disassembled, the poppet was found to have been warped. After contacting the valve manufacturer, replacement poppets were ordered that were made of a harder, more temperature resistant material, Vespel. This was inadvertently temperature tested when a valve body was accidentally run to 200 VDC instead of being pulsed. The temperature of the valve quickly rose to $160{ }^{\circ} \mathrm{C}$ at which point the solenoid itself failed by shorting across turns of wire. When this valve was disassembled the new poppet was found undamaged. Pictures of these poppets and the valve body are shown in Figure 46. This same poppet is currently still operating in a new valve with a new solenoid. It has been pulsing for 
over seven months in this new body, plus one month in the old body. The valve will be considered robust enough for operational use when it completes nine months of continuous pulsing. Nine months is the expected life of the magnetron source, so it could be rebuilt during source refurbishment if needed.

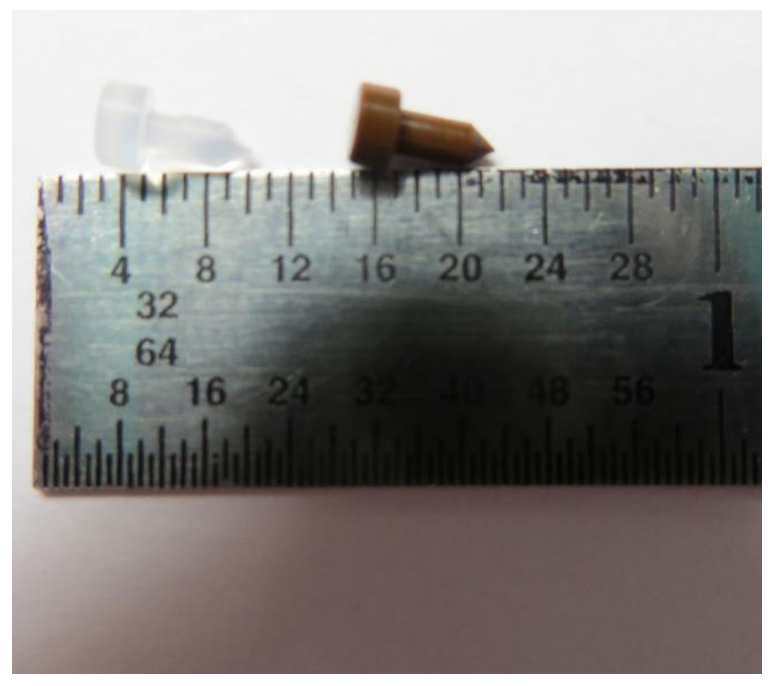

a)

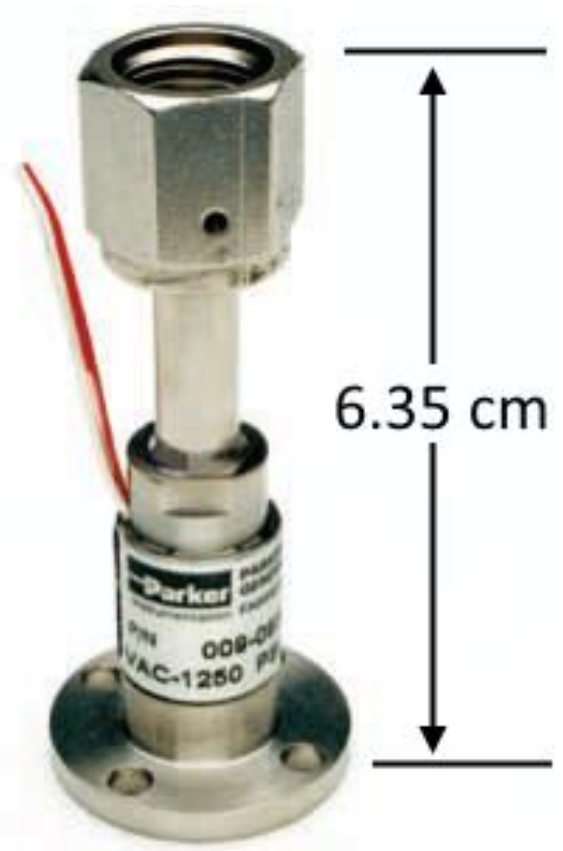

b)

Figure 46: Picture of poppets from the solenoid valves. In (a) on the left is PTFE polymer and on the right is Vespel. Vespel is the material of choice due its resilience at higher temperatures. On the right is the Parker Hannifin Corporation solenoid valve.

\subsubsection{Solid State Extractor Pulser}

When the new style source extractor was designed, a new negative pulsing high voltage pulser was designed. As part of the plan to keep costs low for the conversion, many parts from the old system were reused. The pulser was built around an Eimac 4PR250C tetrode that acted as the switch for the $35 \mathrm{kV}$ that is applied to the extractor cone during the extractor pulse. This tube had been used for years in the old sources. In the old sources, it was pulsed to $18 \mathrm{kV}$ and a tube could be expected to last at least six months before the tube would become emission limited and no long be able to switch fast enough for the extractor pulse. With the change to 
the new sources these tubes now had to pulse to $35 \mathrm{kV}$. This shortened the tube lifetime to 3 months before the emission limited tube would no longer be able to provide the $35 \mathrm{kV}$ in time for the beam request. An example of the tube when healthy and when failing is shown in Figure

47.

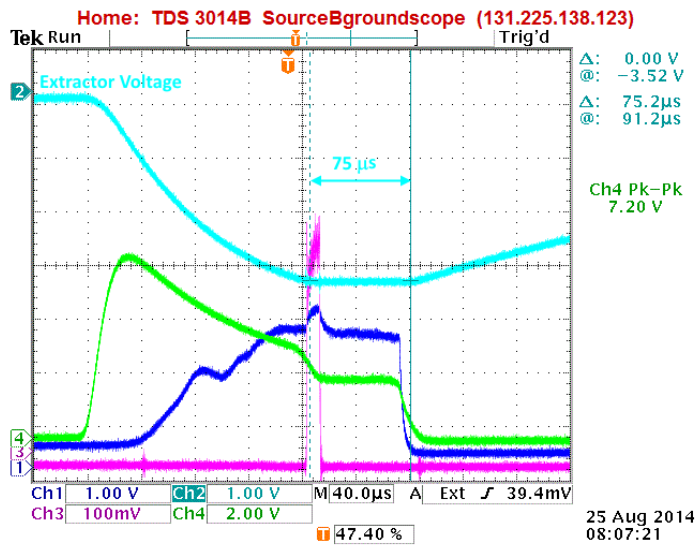

a) Old tube

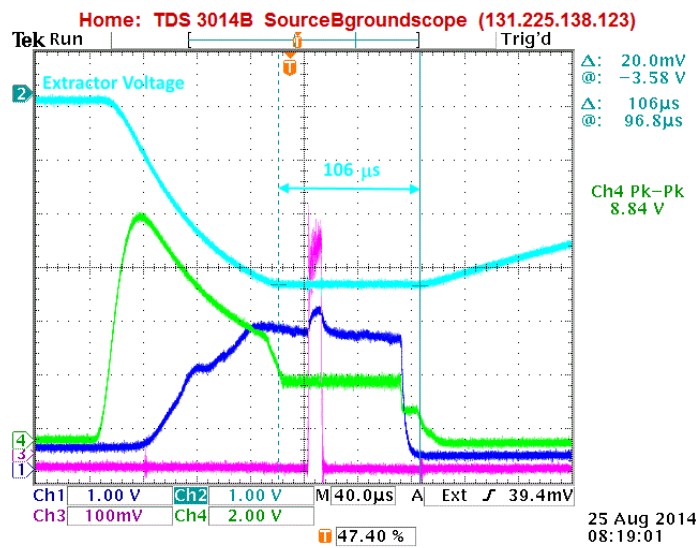

b) New tube

Figure 47: Oscilloscope pictures showing the difference between an emission limited extractor tube (a) and a healthy extractor tube (b). Note the time the extractor takes to reach $35 \mathrm{kV}$ and the time the extractor spends at the $35 \mathrm{kV}$ flattop for beam extraction.

Even when healthy, the tube's long rise time to $35 \mathrm{kV}$ required the arc pulse to be extended by $130 \mu$ s to allow for it to reach full voltage and extract beam that the RFQ could accept, effectively doubling the arc pulse width. It is important to pulse the arc supply prior to the extractor because excessive sparking results from starting the arc while the extractor is already at $35 \mathrm{kV}$.

When testing the sold state switches for the Einzel Lens pulser, it was thought that the same switches could be used for the extractor pulser. The rise time of the solid state switched pulser was $150 \mathrm{~ns}, 1000$ times faster than the tube switches. Initial tests were done using a single bank of the sold state switches that pulsed the extractor and showed that even with the switch not 
properly matched to the extractor, it was able to reach $35 \mathrm{kV}$ in fewer than $50 \mu \mathrm{s}$. As a result more solid state switches were purchased and a new pulser was designed and built.

The new solid state extractor switches were built and then tested in September, 2014. The switches were deliberately made to rise slower than the rise time of the Einzel Lens because such a fast rise time is not needed for the extractor pulser. A $5 \mathrm{k} \Omega$ resister was placed in series to limit the current that could be drawn through the switches that are rated to handle $50 \mathrm{~A}$. Even with this series resistor, the solid state pulser rise time to $35 \mathrm{kV}$ was a short $15 \mu \mathrm{s}$. The switch is opened at the end of the desired pulse width and the current is allowed to flow through the resistive elements in the circuit. As the extractor is pulsed once every $67 \mathrm{~ms}$, the pulser has plenty of time to bleed down.

The difference in rise times between the tube and solid state switched pulsers is $115 \mu$ s and is shown in Figure 48. This allows for a much longer beam flattop than needed, or allows for the arc pulse to be shortened. The arc pulse width is $230 \mu$ s so this would allow for a reduction of $50 \%$ to the arc pulse width. A change this big requires corresponding adjustments to the source body heater and the cesium supply heaters as the shorter arc pulse width will provide less heat to the system. The arc discharge is sensitive to temperature and requires the cathode and source body to maintain a nominal temperature to remain stable. If the temperature drops below this level the arc pulse will falter and it will take substantial time and effort to restore the source to operation. Slowly changing the arc pulse width over the course of several days for a source in the test stand required no changes to maintain a stable arc discharge. In operations, this change will be made even more slowly in the operational sources because their stability is of utmost importance for providing stable beam to FNAL HEP experiments. 


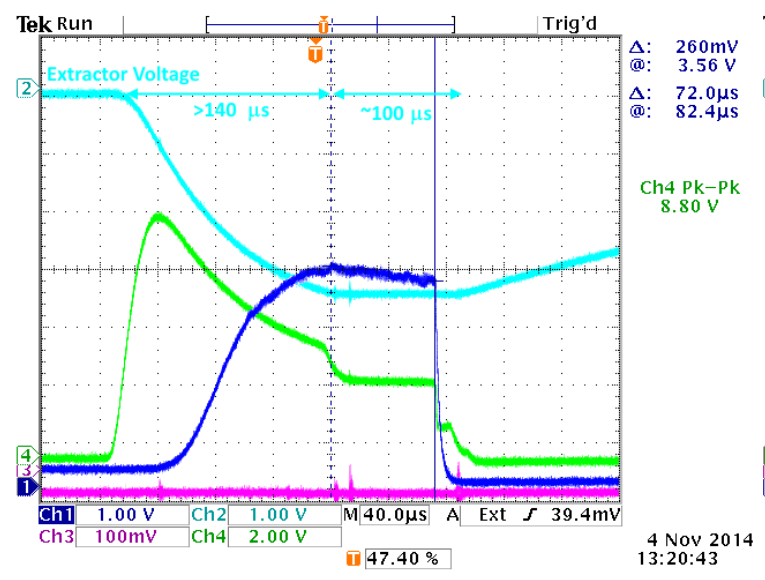

a) Tube extractor pulser

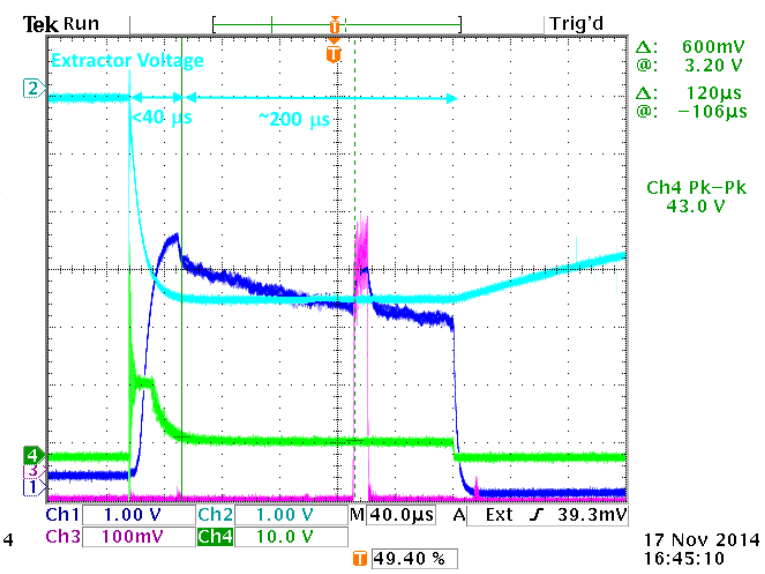

b) Solid state extractor pulser

Figure 48: Oscilloscope plots comparing tube switched extractor pulse (a) and solid state switched extractor pulse (b). The solid state switch takes $10 \%$ of the time to reach the $35 \mathrm{kV}$ flattop and allows for a beam flattop of $200 \mu \mathrm{s}$ versus $100 \mu \mathrm{s}$ with the tube pulser.

One consequence of this shorter arc pulse width is a lower duty factor for the source operation. The drop in duty factor could lead to longer operational periods and a source lasting longer than the current 6-9 months. The reduced arc pulse should also require less cesium to maintain the optimal layer and further extend the lifetime of the $5 \mathrm{~g}$ ampules used in the sources.

\section{Conclusions}

The commissioning and installation of the new RIL front end for the FNAL Linac was a long and arduous task, continuing long after the system was installed. Much of the ion source development only occurred after the RIL was operational, and continues today. The sources have evolved over time and will no doubt continue to do so for years to come.

The initial goal was to "do no harm" at the minimum and be able to match the beam qualities that were provided by the Cockcroft-Walton sources. At first glance it might seem that the RIL did not meet this expectation. Although the beam into Linac is $29 \mathrm{~mA}$ which is reduced 
from $49 \mathrm{~mA}$ from the Cockcroft Waltons, much of this $49 \mathrm{~mA}$ of beam was lost in the first RF tank in Linac. Linac used to provide $35 \mathrm{~mA}$ of beam to Booster, but with the RIL it can only provide (27-28) mA. This decrease in intensity is compensated by increasing the number of turns in the Booster. Increasing the number of turns mean that Booster layers successive $2.2 \mu \mathrm{s}$ lengths of beam until the desired intensity has been accumulated. Efficiency wise Linac now accelerates $94 \%$ of the beam injected into it, whereas previously it provided $72 \%$. This is a huge jump in efficiency and means that less beam is lost in Linac which translates to less irradiation of components.

Work is ongoing to further improve the RIL performance and will continue into the foreseeable future. Neutralization tests in the LEBT has thus far used hydrogen. The next step will be to inject nitrogen gas into the LEBT to look for more effective neutralization. This should help increase the RIL efficiency and increase Linac intensity.

A new test stand has been built that fixes the vacuum level problems encountered in the previous test stand. This will allow us to better understand the effects of the cathode volume changes and nitrogen doping. A new gas mixing scheme is being designed that will allow us to have better control over the nitrogen fraction in the source gas mixture as well. Either of these methods could lead to a less noisy arc discharge in the source and hopefully lead to a less noisy and thus more stable beam output. A more stable source beam output will benefit all of the downstream machines and the Fermilab HEP program as a whole. 


\section{Acknowledgement}

Operated by Fermi Research Alliance, LLC under Contract No. De-AC02-07CH11359 with the United States Department of Energy.

\section{References}

[1] C.W. Schmidt \& C.D. Curtis, "A 50-mA Negative Hydrogen Ion Source", IEEE Proc. Nucl. Sci., NS-26, pg. 4120-4122, 1972.

[2] C.Y. Tan, D.S. Bollinger, K.L. Duel, P.R. Karns, M.J. Kucera, J.R. Lackey, J.F. Larson, W.A. Pellico, E.A. Peoples-Evans, V.E. Scarpine, C.W. Schmidt, B.A. Schupbach, R.E. Tomlin \& A.K. Triplett. The $750 \mathrm{keV}$ RFQ injector upgrade. Technical Report Beams-doc-3646-v16, Fermilab, 2013.

[3] J.R. Lackey, D.S. Bollinger, P.R. Karns, C.Y. Tan. A novel low energy fast chopper for Hinjectors. Nucl. Instrum. Meth. A 701: 194-200, 2013.

[4] D. Raparia, et al, "Results of LEBT/MEBT Reconfiguration at BNL 200 MeV Linac", Part. Acc. Conf. Proc. 2009.

[5] S.S. Kurennoy, R.W. Garnett, L.J. Rybarcyk. Electromagnetic and multi-particle beam dynamics modeling of 4-rod RFQs. In Proceedings of IPAC2013, 2013.

[6] J. S. Schmidt, B. Koubek, A. Schempp, C. Y. Tan, D. S. Bollinger, K. L. Duel, P. R. Karns, W. A. Pellico, V. E. Scarpine, B. A. Schupbach, and S. S. Kurennoy. Investigations of the output energy deviation and other parameters during commissioning of the four-rod radio frequency quadrupole at the Fermi National Accelerator Laboratory. Phys. Rev. ST Accel. Beams 17, 030102, 2014. 
[7] M. Okamura, H. Yamauchi. Effects of grids in drift tunes. In Proceedings of IPAC2012, 2012

[8] D.S. Bollinger, P.R. Karns, C.Y Tan. A Cookbook for building a high current dimpled Hmagnetron source for accelerators. IEEE Trans. Plasma Science. Submitted for publication.

[9] H. Zhang, lon Sources, ISBN 3-540-65747-9 Springer. 341, 1999.

[10] J.D. Cobine. Field-intensified ionization and breakdown of gases. In Gaseous conductors theory and engineering applications, chapter VII. Dover, 1958.

[11] Yu.I. Belchenko, G.I. Dimov and V.G. Dudnikov, Nucl. Fusion 14 (1974) 113, 1974.

[12] J. G. Alessi and Th. Sluyters. Regular and asymmetric negative ion magnetron sources with grooved cathodes. Rev. Sci. Instrum., 51(12), 1980.

[13] K. Wiesemann. Some physical aspects of H- sources. Proc. Symp. On the Production and Neutralization of Negative lons and Beams, BNL 50272, 97, 1977.

[14] D.S. Bollinger, P.R. Karns, C.Y. Tan. Reduction of Beam Current Noise in the FNAL Magnetron Ion Source. In AIP Conf. Proc. 1655, 070002, 2015.

[15] V. Dudnikov, R. P. Johnson. High brightness H- surface plasma sources. In Proceedings of IPAC'10, 2010.

[16] H. Vernon Smith Jr. Emission spectroscopy of the $4 \mathrm{X}$ source discharge with and without N2 gas. AT-10 TECHNICAL NOTE 89-07, Los Alamos National Laboratory, April 1989. 


\section{A. Appendices for Mathematica Programs}

\section{A.1 LEBT Beam line Model}

Calibrating the test line (27 Dec 2011)

I'm going to change tactics here and assume that there *is* a lens from gas focusing at the end of solenoid2. This is a revisit of my old idea to see if it works here

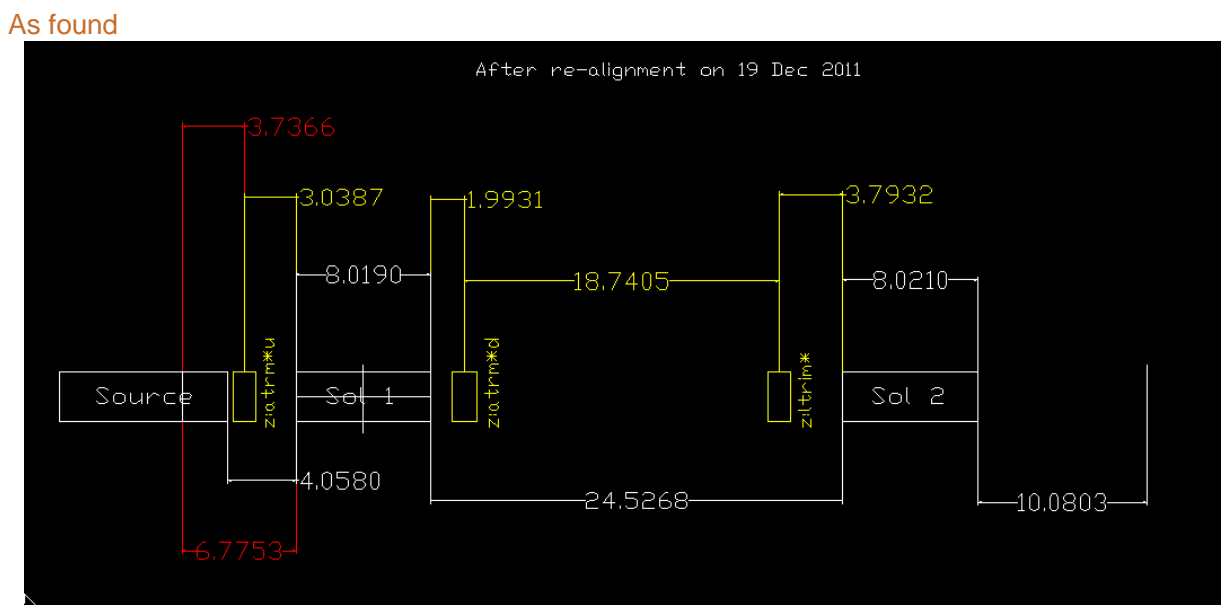

Solenoid effective

After correcting solenoid 2, 19 Dec 2011

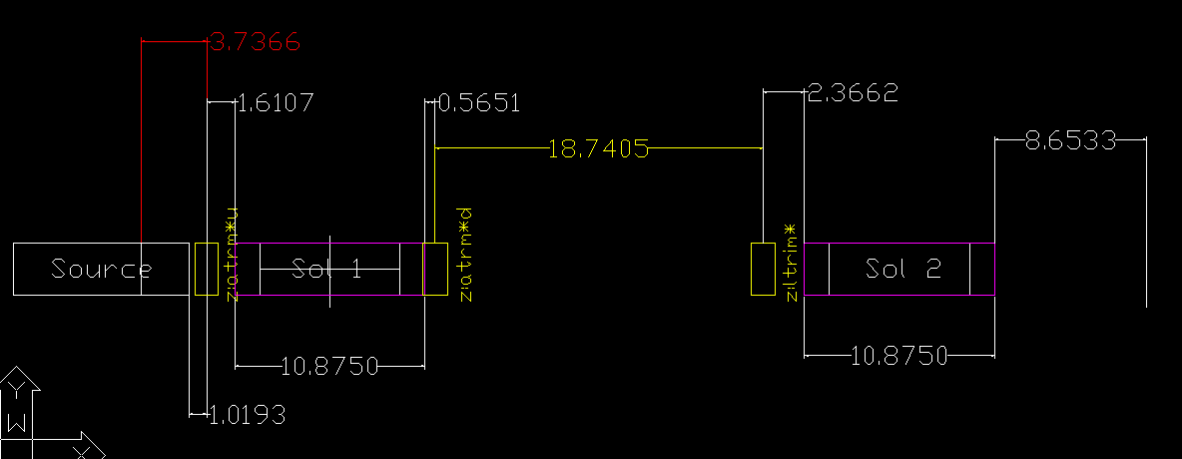

Solenoid 1 set to $466 \mathrm{~A}$ and solenoid 2 is set to 418 amps. NOT paraxial beam.

\section{Constants}

$\mathrm{c}=310^{8} ;(* \mathrm{~m} / \mathrm{s} *)$

$\mathrm{p}=8.110^{6} ;\left(* \mathrm{eV} / \mathrm{c}^{*}\right)$

inch2m $=2.54 \quad 10^{-2}$;

\section{Measured Bz}

The data is from PSSA001-0 excitation and z scans.xlsx. I extracted out the $z$ vs bz ${ }^{2}$ column to produce bz2.dat. This data has been taken at $400 \mathrm{~A}$.

bz2 = ReadList [" /expt/rfq/math/bz2.dat", \{Number, Number\}];

Convert $\mathrm{z}$ to metres and make $\mathrm{Bz}$ from $\mathrm{Bz}^{2}$

bzm $=$ Table $[\{b z 2[[i, 1]]$ inch2m, Sqrt $[b z 2[[i, 2]]]\},\{i$, Length $[b z 2]\}] ;$

ListPlot[bzm, Joined-> True, AxesLabel-> \{"z (m)", "Bz(T)"\}] 


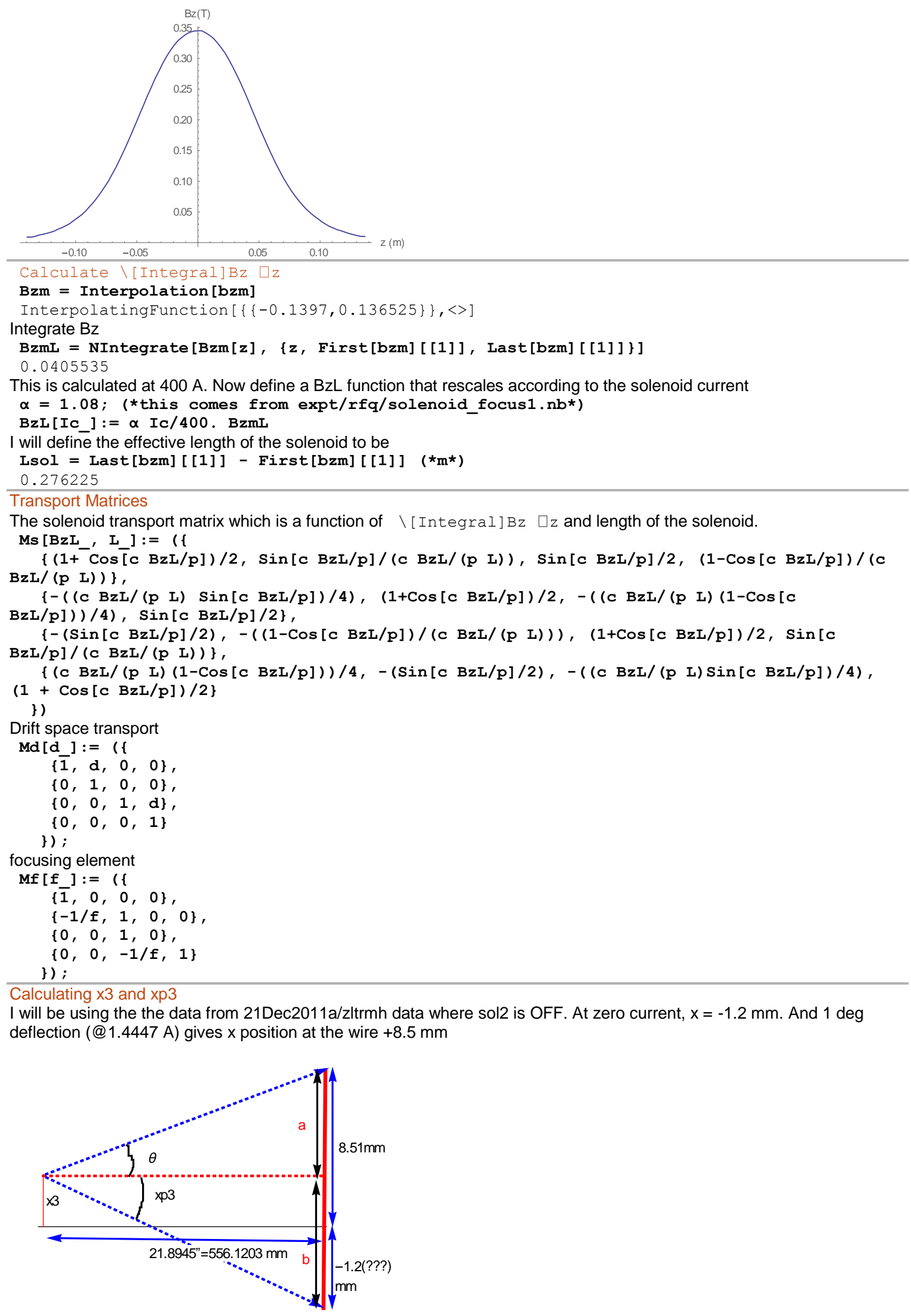




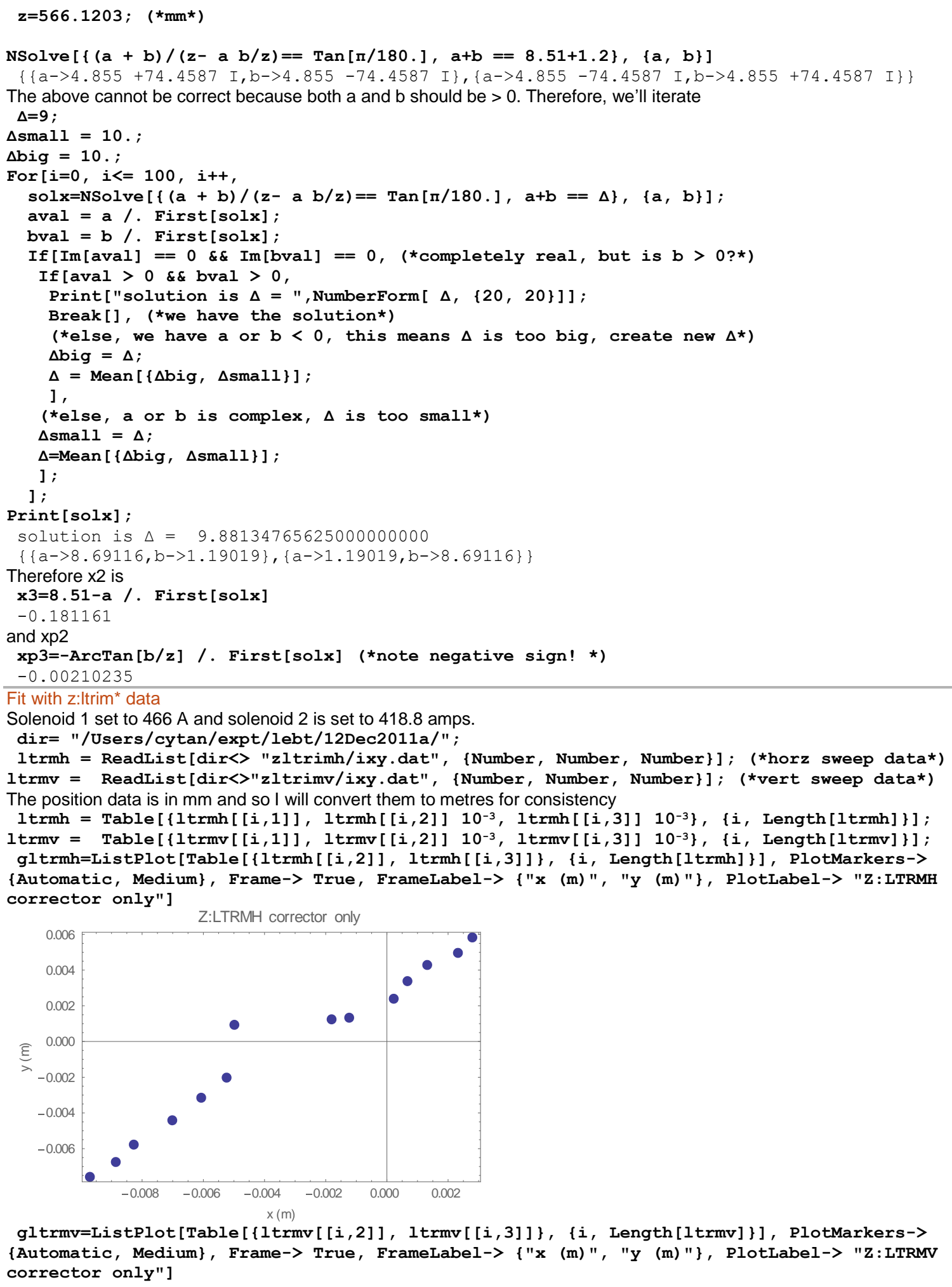

gltrmv=ListPlot[Table $[\{1 \operatorname{trmv}[[i, 2]], 1 \operatorname{trmv}[i, 3]]\},\{i$, Length[1trmv]\}], PlotMarkers-> \{Automatic, Medium\}, Frame-> True, Framelabel-> \{"x (m)", "y (m)"\}, PlotLabel-> "z:LTRMV corrector only"] 


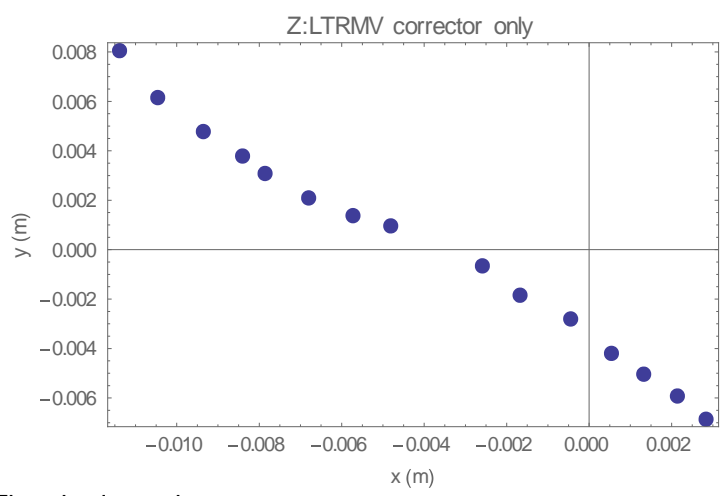

Fit to horizontal scan

fzltrmh = Fit $[\operatorname{Table}[\{1 \operatorname{trmh}[[i, 2]], 1 \operatorname{trmh}[[i, 3]]\},\{i, \operatorname{Length}[1 \operatorname{trmh}]\}],\{1, \mathbf{x}\}, \mathbf{x}]$

$0.00292914+1.01035 x$

$0.00292914+1.01035 \times$

$0.00292914+1.01035 \times$

Show [gltrmh, Plot[fzltrmh, $\{x,-0.01,0.01\}$, PlotStyle-> Red] ]

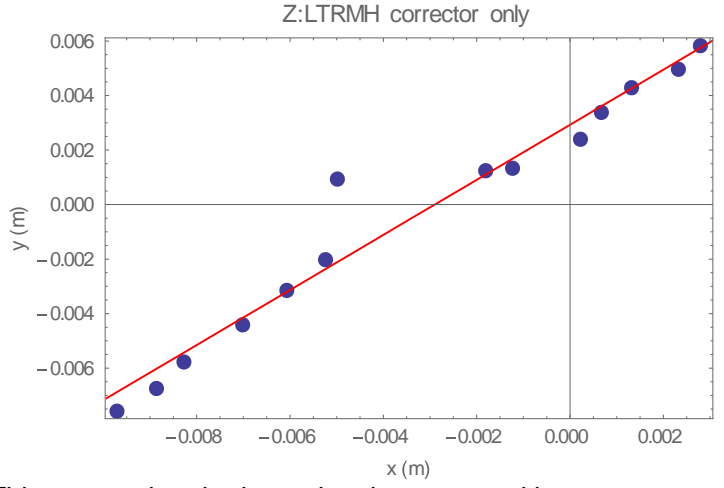

This means that the beam has been rotated by

$\theta \mathrm{h}=$ ArcTan [Coefficient [fzltrmh, x] $]$ 180/n (* deg*)

45.295

The rotation formula for going through a solenoid is

$\phi=-q B z L /(2 p)$ in SI units

$=-\mathrm{c} \mathrm{BzL} /(2 \mathrm{p})$ in $\mathrm{eV}$ units

Note: we have no negative sign because the B-field here is anti-parallel to the beam

$\phi h=c I B z L /(2$ p) $180 / \pi$

1061.03 IBzL

sol=Solve $[\phi \mathrm{h}==\theta \mathrm{h}, \mathrm{IBzL}]$

$\{\{$ IBzL->0.0426895\}\}

sol2=Solve [BzL[Isol2] == (IBzL/. First[sol]), Isol2]

$\{\{$ Isol2->389.878\} \}

Is2 = Isol2/.First [sol2]

389.878

Correctors

From the 21 Dec 2011a measured data, the $z$ :Itrimh $0.692168 \mathrm{deg} / \mathrm{A}$.

zltrmh [Ic_] $:=(\{$

$\{0\}$

$\{0.692168 \mathrm{~m} / 180 . \mathrm{Ic}\}$,

$\{0\}$,

$\{0\}$

\});

Note : if zltrmh is the following, the fits are actually much better. However, the measurements really want the calibration to be $0.69 \mathrm{deg} / \mathrm{V}$. It was measured twice.

(*zltrmh [Ic_] : $=10$

$0.422771 \pi / 1 \overline{80}$. Ic

)$; *)$

and from 09 Dec 2011 z:Itrmv is $0.422771 \mathrm{deg} / \mathrm{A}$

(*zltrmv[Ic_] := $(0$ 


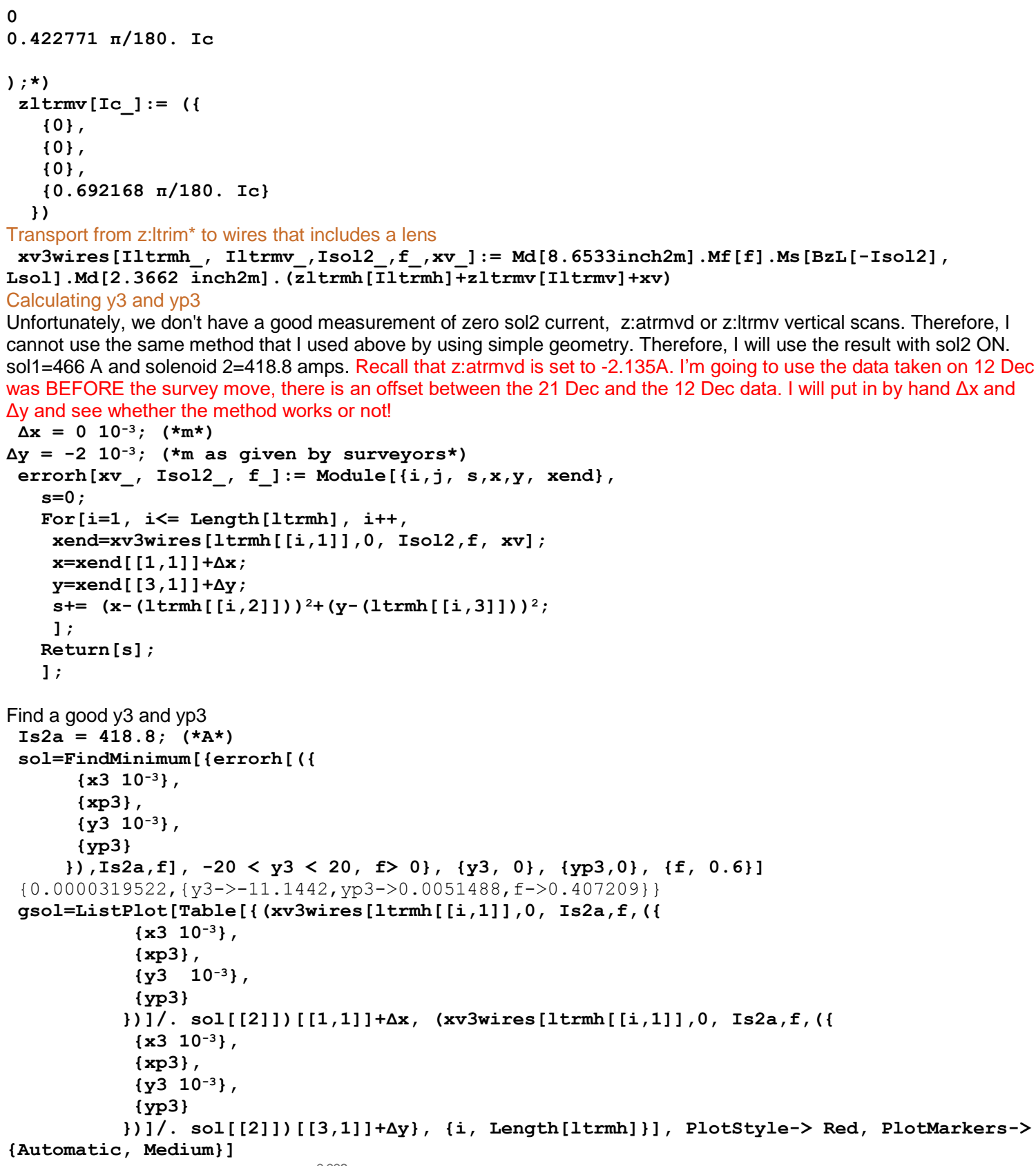




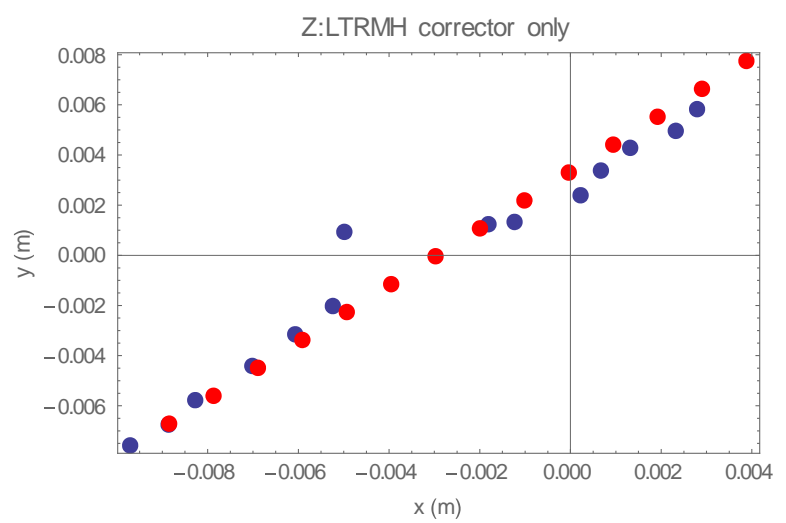

$\mathrm{OK}$, the size and slope fits but it is surprising that the solenoid is so much stronger, in fact about a factor of 1.38 times stronger! Check z:Itrmv

gsolv1=ListPlot $[\mathrm{Tab} l e[\{(\mathrm{xv} 3$ wires $[0,1 \operatorname{trmv}[[i, 1]], \operatorname{Is} 2 a, f,(f$

$\left\{x 310^{-3}\right\}$,

$\{\mathrm{xp} 3\}$

$\left\{y^{3} 10^{-3}\right\}$

$\{\mathrm{yp} 3\}$

\}) $] /$. sol $[[2]])[[1,1]]+\Delta x, \quad(x v 3 w i r e s[0,1 \operatorname{trmv}[[i, 1]]$, Is $2 a, f,(\{$

$\left\{\times 310^{-3}\right\}$,

$\{\mathrm{xp} 3\}$

$\left\{\mathrm{y}^{3} 10^{-3}\right\}$,

$\{\mathrm{yp} 3\}$

\}) $]$ /. sol[[2] $][[3,1]]+\Delta y\},\{i$, Length [1trmv] $\}]$, PlotStyle-> Red, PlotMarkers-> \{Automatic, Medium\}]

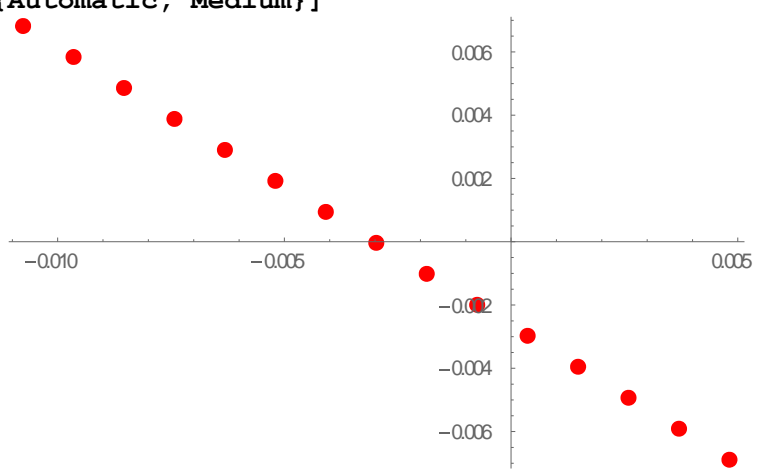

Show [gltrmv, gsolv1, PlotRange-> All]

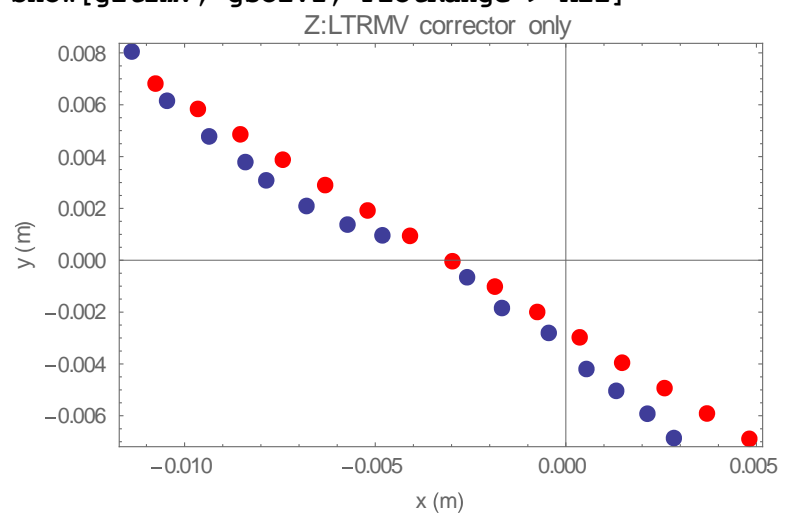

Redo using both z:Itrmh and z:Itrmv data

errorhv $\left[x_{-}\right.$, Isol2_, $\left.f_{-}\right]:=\operatorname{Module}[\{i, j, s, x, y$, xend $\}$,

$\mathrm{s}=0$;

For $[i=1, i<=$ Length $[1$ trmh $], i++$,

xend=xv3wires $[1 \operatorname{trmh}[[i, 1]], 0$, Isol2, $f, x v]$;

$\mathbf{x}=\mathbf{x e n d}[[1,1]]+\Delta \mathbf{x}$;

$y=x e n d[[3,1]]+\Delta y$

$s+=(x-(1 \operatorname{trmh}[[i, 2]]))^{2}+(y-(1 \operatorname{trmh}[[i, 3]]))^{2}$; 
] ;

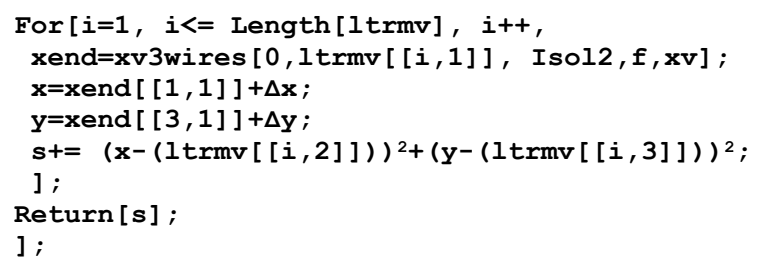

\}) $] /$. sol $[[2]])[[3,1]]+\Delta y\},\{i$, Length $[1$ trmh $]\}]$, PlotStyle-> Red, PlotMarkers-> \{Automatic, Medium\}]

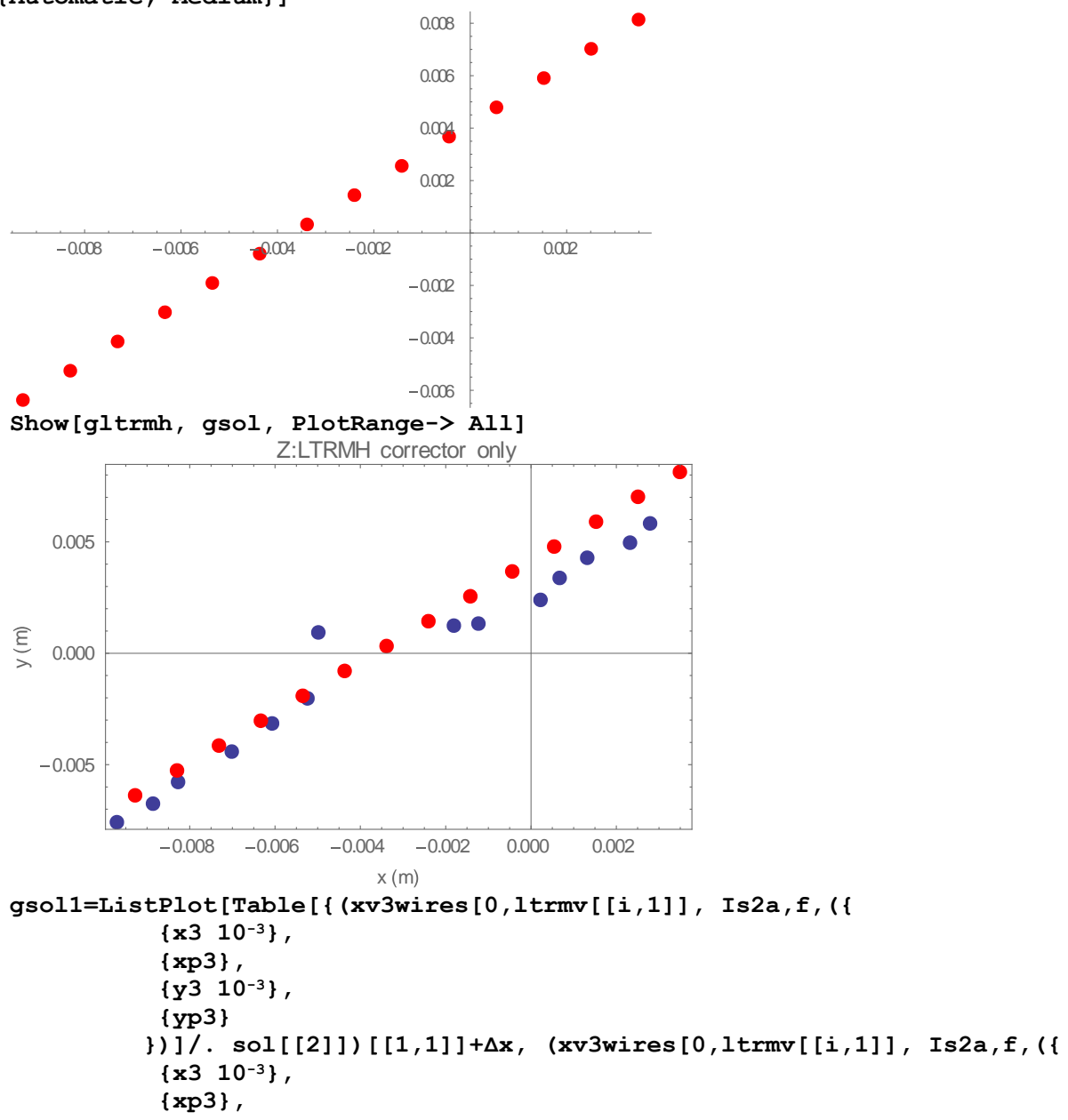




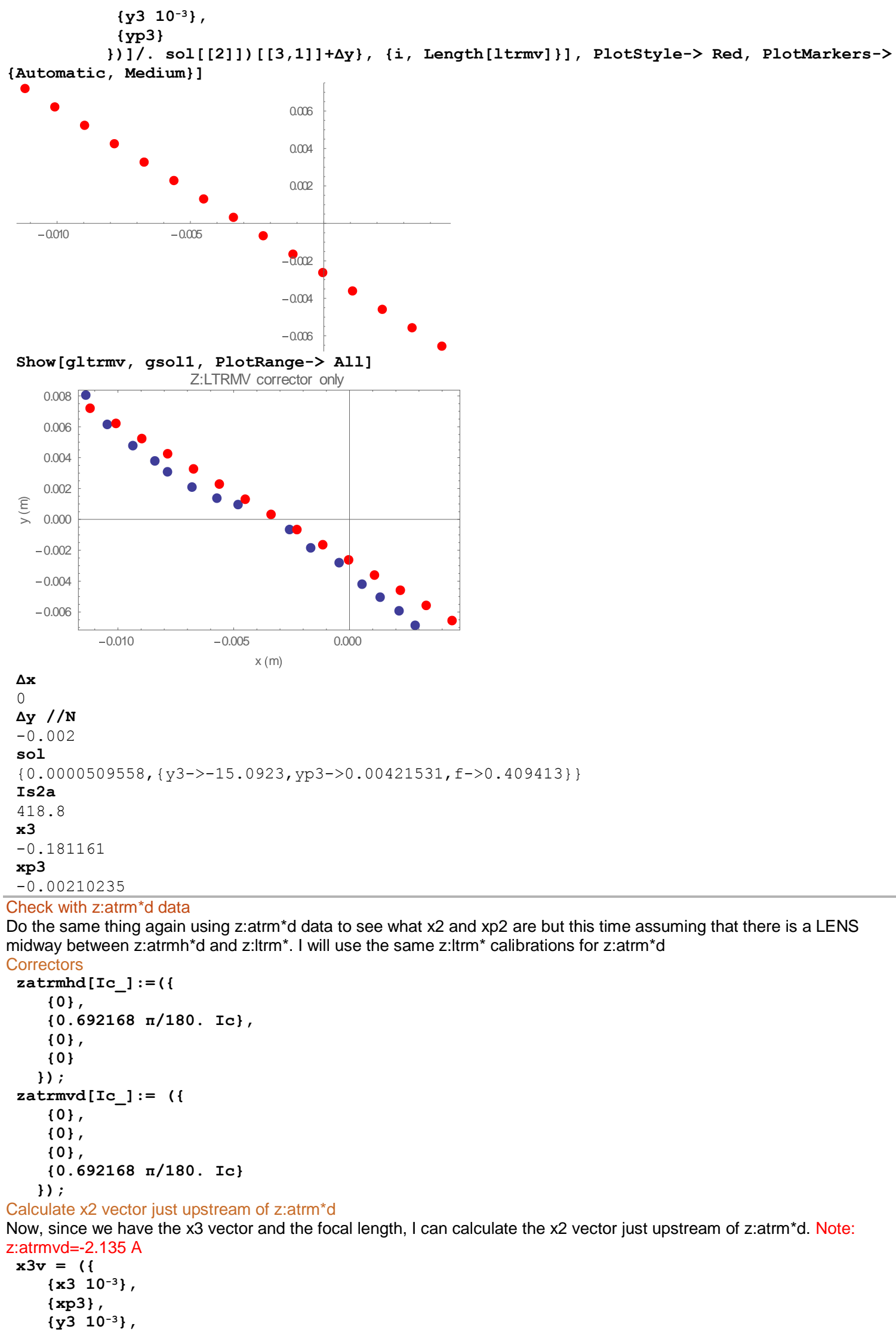

$-0.004$

$-0.006$

Show [gltrmv, gsol1, PlotRange-> All]

$\Delta \mathbf{x}$

0

$\Delta \mathrm{y} / / \mathrm{N}$

$-0.002$

sol

$\{0.0000509558,\{y 3->-15.0923, y p 3->0.00421531, f->0.409413\}\}$

Is $2 \mathrm{a}$

418.8

x3

$-0.181161$

xp3

$-0.00210235$

Check with z:atrm*d data

Do the same thing again using $z$ :atrm ${ }^{*} d$ data to see what $x 2$ and $x p 2$ are but this time assuming that there is a LENS midway between $z$ :atrmh ${ }^{*} d$ and $z: I t r{ }^{*}$. I will use the same $z$ :Itrm* calibrations for $z$ :atrm*d

Correctors

Now, since we have the $x 3$ vector and the focal length, I can calculate the $x 2$ vector just upstream of $z$ :atrm*d. Note: z:atrmvd $=-2.135 \mathrm{~A}$

$\mathrm{x} 3 \mathrm{v}=(1$

$\left\{\times 310^{-3}\right\}$

$\{\mathrm{xp} 3\}$,

$\left\{y^{3} 10^{-3}\right\}$, 
$\{$ yp3 $\}$

\}) /. sol [ [2] ]

$\{\{-0.000181161\},\{-0.00210235\},\{-0.0150923\},\{0.00421531\}\}$

Note: that there is a corrector setting of $z$ :atrmvd=-2.135A.

sol2 = NSolve $[$ Md $[18.7405$ inch2m $] .($ zatrmvd $[-2.135]+(f$

$\{x 2\}$,

$\{x p 2\}$,

$\left\{\mathrm{y}^{2}\right\}$,

$\{$ yp2 $\}$

\}) ) $==x 3 v,\{x 2, x p 2, y 2, y p 2\}]$

$\{\{\mathrm{x} 2->0.000819577, \mathrm{xp} 2->-0.00210235, \mathrm{y} 2->-0.0170988, \mathrm{yp} 2->0.0300074\}\}$

$x 2 v=(t$

$\{x 2\}$,

$\{x p 2\}$,

$\left\{\mathrm{y}^{2}\right\}$,

\{yp2\}

\}) /. First [sol2]

$\{\{0.000819577\},\{-0.00210235\},\{-0.0170988\},\{0.0300074\}\}$

Transport from zatrm*d to wire including lens at end of solenoid

atrmd2wire [ Iatrmhd_, Iatrmvd_, Isol2_,f_, xv_] := Md [8.6533inch2m].Mf [f].Ms [BzL [-Isol2],

Lsol] . Md [ (18.7405+2.3662) inch2m] . (zatrmhd [ Iatrmhd] +zatrmvd [ Iatrmvd]+xv)

z:atrm*d data

atrmhd = ReadList [dir<> "zatrmhd/ixy.dat", \{Number, Number, Number\}]; (*horz sweep data*)

atrmvd = ReadList [dir<>"zatrmvd/ixy.dat", \{Number, Number, Number\}]; (*vert sweep data*)

The position data is in $\mathrm{mm}$ and so I will convert them to metres for consistency

atrmhd $\left.=\operatorname{Table}\left[\{\operatorname{atrmhd}[i, 1]], \operatorname{atrmhd}[[i, 2]] 10^{-3}, \operatorname{atrmhd}[i, 3]\right] 10^{-3}\right\},\{i$,

Length [atrmhd] \}] ;

atrmvd $\left.=\operatorname{Table}[\{\operatorname{atrmvd}[i, 1]], \operatorname{atrmvd}[i, 2]] 10^{-3}, \operatorname{atrmvd}[[i, 3]] 10^{-3}\right\},\{i$,

Length [atrmvd] \}] :

Plot them out

gatrmhd=ListPlot $[$ Table $[\{$ atrmhd $[i, 2]]$, atrmhd $[i, 3]]\},\{i$, Length $[$ atrmhd $]\}]$,

PlotMarkers-> \{Automatic, Medium\}, Frame-> True, FrameLabel-> \{ $x$ (m) ", "y (m) "\},

PlotLabel-> "z:ATRMHD corrector only"]

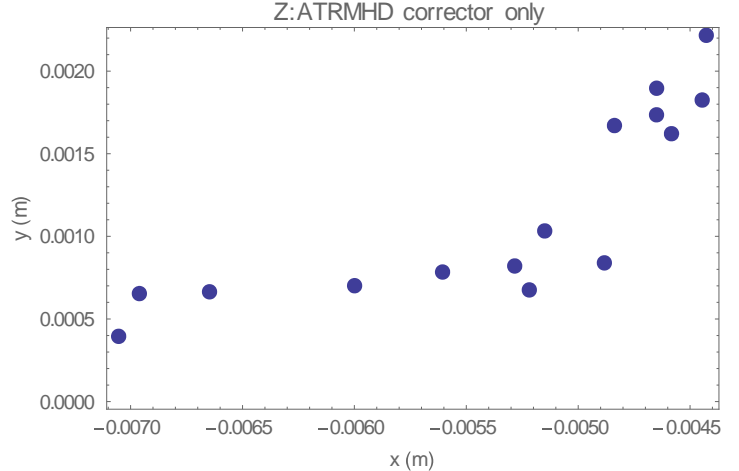

Hmmm, looks like only data above > 0.0054 make any sense because y does not seem to move otherwise. So, get this subset out

atrmhd1 $=\{\}$;

For $[i=1, i<=$ Length $[$ atrmhd], $i++$,

If $[$ atrmhd $[i, 2]]>-0.0054$,

atrmhd1 = Join [atrmhd1, \{atrmhd[[i]]\}];

];

] ;

gatrmhd1=ListPlot $[$ Table $[\{\operatorname{atrmhd} 1[i, 2]], \operatorname{atrmhd}[[i, 3]]\},\{i, \operatorname{Length}[$ atrmhd1 $]\}]$,

PlotMarkers-> \{Automatic, Medium\}, Frame-> True, FrameLabel-> \{"x (m) ", "y (m)"\},

PlotLabel-> "z:ATRMHD corrector only"] 


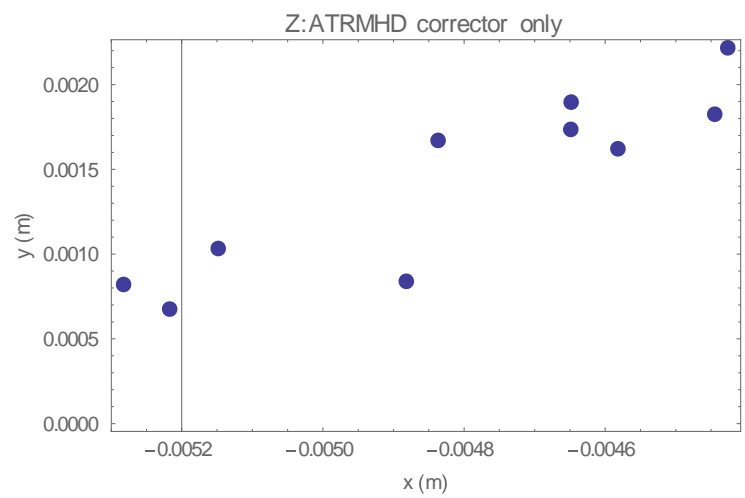

gatrmvd=ListPlot [Table $[\{$ atrmvd $[i, 2]]$, atrmvd[[i,3] $\},\{i$, Length $[$ atrmvd] $\}]$ PlotMarkers-> \{Automatic, Medium\}, Frame-> True, FrameLabel-> $\{$ "x (m) ", "y (m) "\}, PlotLabel-> "Z:ATRMVD corrector only"]

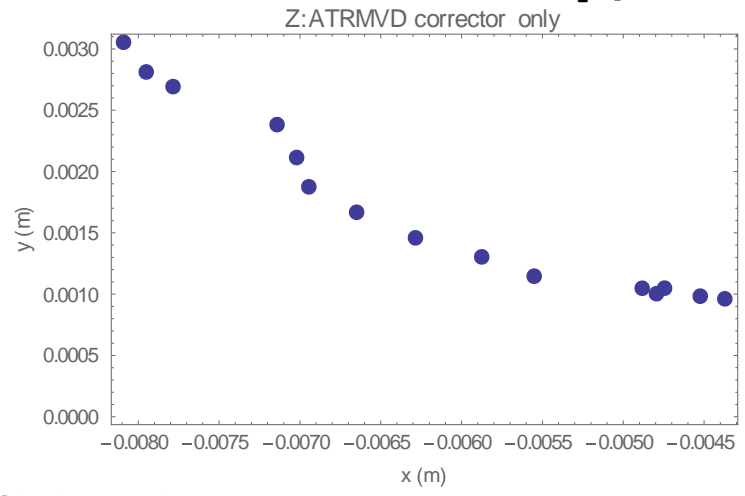

Check atrmvd

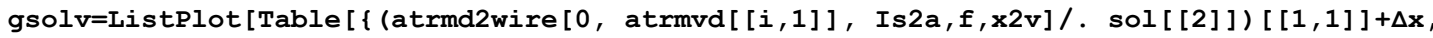
(atrmd2wire [0, atrmvd [ $[i, 1]], \operatorname{Is} 2 a, f, x 2 v] / . \operatorname{sol}[[2]])[[3,1]]+\Delta y\},\{i$, Length $[a t r m v d]\}]$, PlotStyle-> Red, PlotMarkers-> \{Automatic, Medium\}]

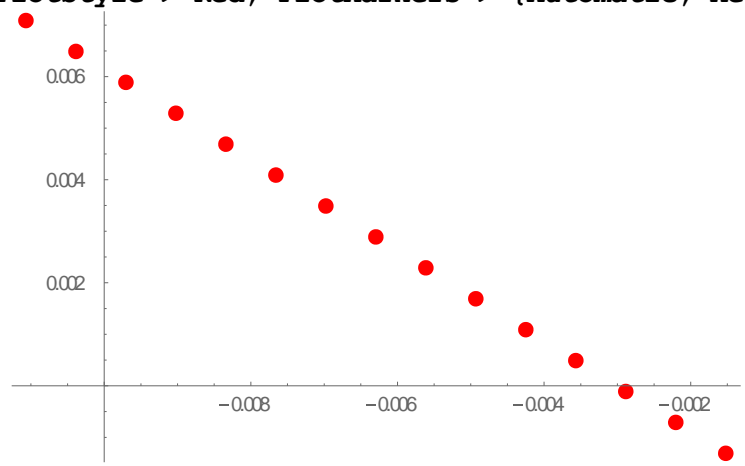

Show [gatrmvd, gsolv, PlotRange-> All]

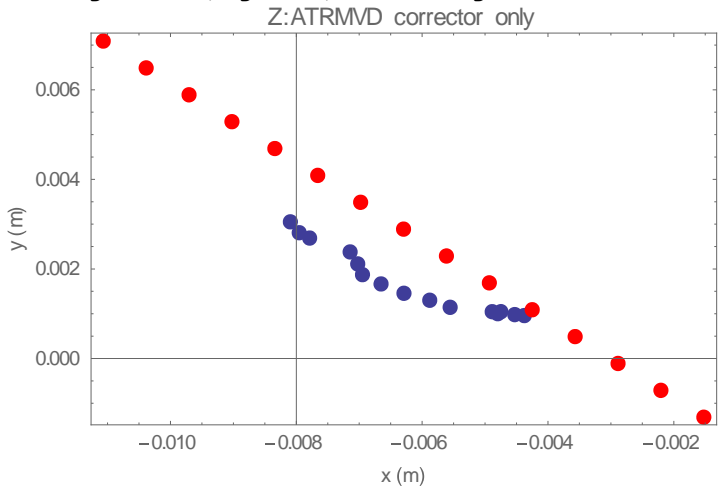

gsol=ListPlot $[$ Table $[\{$ (atrmd2wire $[$ atrmhd $[i, 1]],-2.135$, Is $2 a, f, x 2 v] /$. sol $[[2]])[[1,1]]+\Delta x$, (atrmd2wire [atrmhd $[i, 1]],-2.135$, 
Is $2 a, f, x 2 v] / . \operatorname{sol}[[2]])[[3,1]]+\Delta y\},\{i$, Length [atrmhd] $\}]$, PlotStyle-> Red, PlotMarkers-> \{Automatic, Medium\}]
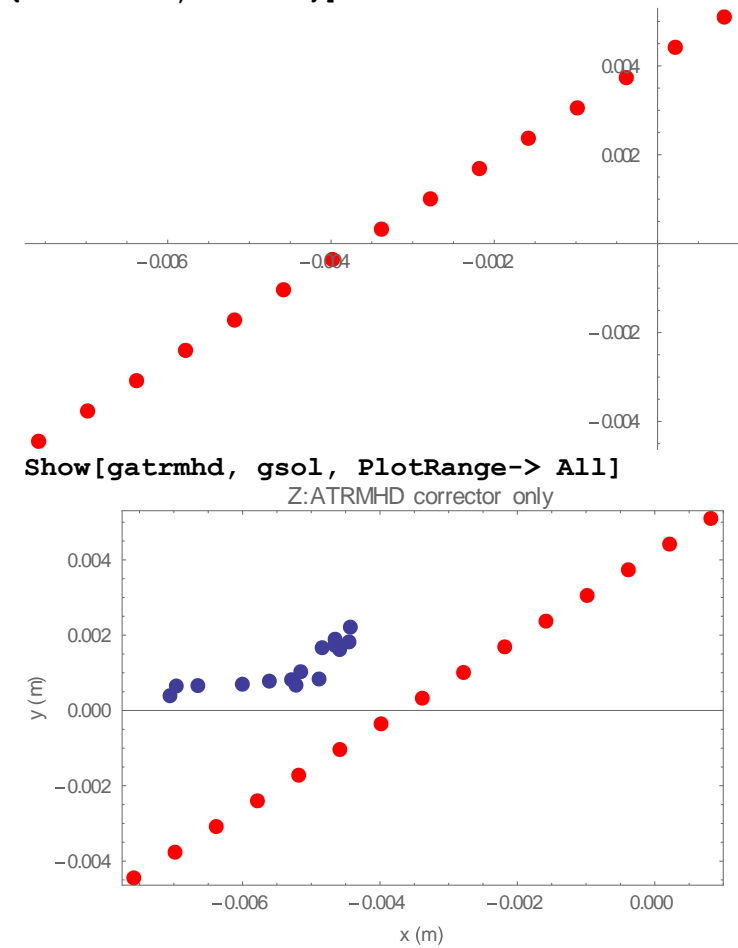

Redo using both z:atrmhd and z:atrmvd data

aerrorhv $\left[x_{-}\right.$, Isol2_, $\left.f \_\right]:=\operatorname{Module}[\{i, j, s, x, y$, xend $\}$,

$\mathrm{s}=0$;

For $[i=1, i<=$ Length $[$ atrmhd1], $i++$,

xend=atrmd2 wire $[$ atrmhd1 $[[i, 1]],-2.135$, Isol2, $f, x v]$;

$\mathbf{x}=\mathbf{x e n d}[[1,1]]+\Delta \mathbf{x}$;

$y=x e n d[[3,1]]+\Delta y$

$\left.s+=(x-(\operatorname{atrmhd} 1[[i, 2]]))^{2}+(y-(\operatorname{atrmhd} 1[i, 3]])\right)^{2} ;$

] ;

For $[i=1, i<=$ Length [atrmvd], $i++$,

xend=atrmd2 wire $[0$, atrmvd $[i, 1]]$, Isol2, $f, x v]$;

$\mathbf{x}=\mathbf{x e n d}[[1,1]]+\Delta \mathbf{x}$;

$y=x e n d[[3,1]]+\Delta y$

$s+=(x-(\operatorname{atrmvd}[i, 2]]))^{2}+(y-(\operatorname{atrmvd}[[i, 3]]))^{2}$;

] ;

Return [s] ;

] ;

Just using the setting and the fits actually look good!

sol=FindMinimum $[\{\operatorname{aerrorhv}[x 2 v, I s 2 a, f], f>0\},\{f, 0.6\}]$

$\{0.0000280293,\{f->0.306222\}\}$

gsol=ListPlot [Table [ $\{$ (atrmd2wire [atrmhd1 $[[i, 1]],-2.135$, Is $2 a, f, x 2 v] /$

sol [ [2] ]) $[[1,1]]+\Delta x$, (atrmd2wire [atrmhd1 $[i, 1]],-2.135$, Is $2 a, f, x 2 v] /$.

sol $[[2]])[[3,1]]+\Delta y\}$, $\{i$, Length $[$ atrmhd1 $]\}]$, PlotStyle-> Red, PlotMarkers-> \{Automatic, Medium\} ] 


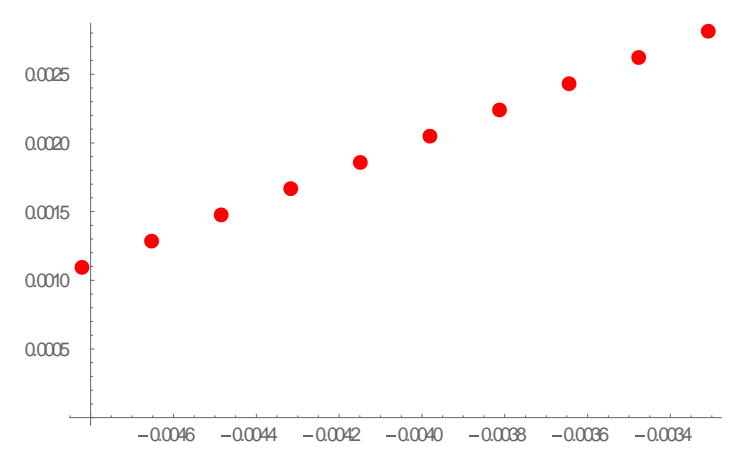

Show [gatrmhd1, gsol, PlotRange-> All]

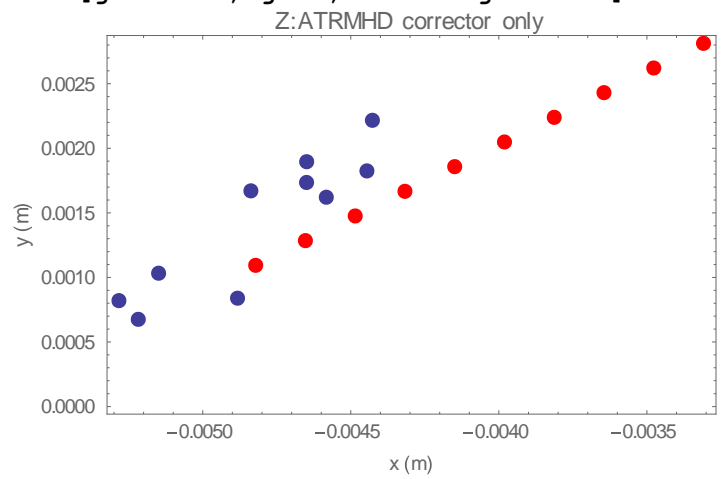

gsol1=ListPlot [Table $[\{(\operatorname{atrmd} 2$ wire $[0, \operatorname{atrmvd}[[i, 1]], \operatorname{Is} 2 a, f, x 2 v] / . \operatorname{sol}[[2]])[[1,1]]+\Delta \mathbf{x}$, (atrmd2wire $[0, a \operatorname{trmvd}[[i, 1]], \operatorname{Is} 2 a, f, x 2 v] /$. sol $[[2]])[[3,1]]+\Delta y\},\{i$, Length $[a t r m v d]\}]$, PlotStyle-> Red, PlotMarkers-> \{Automatic, Medium\}]

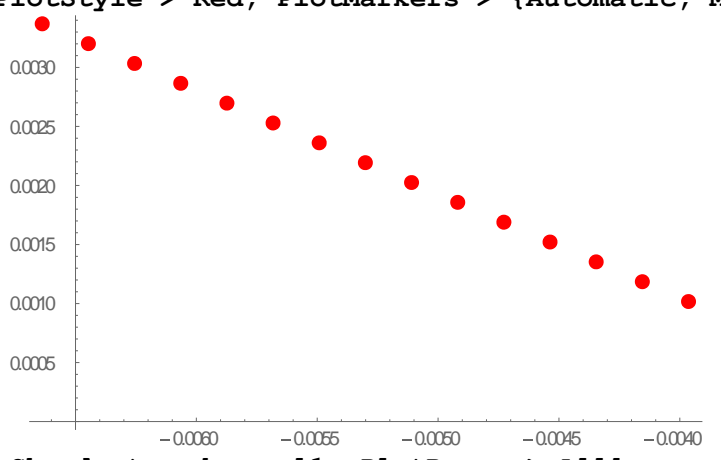

Show [gatrmvd, gsoll, PlotRange-> All]

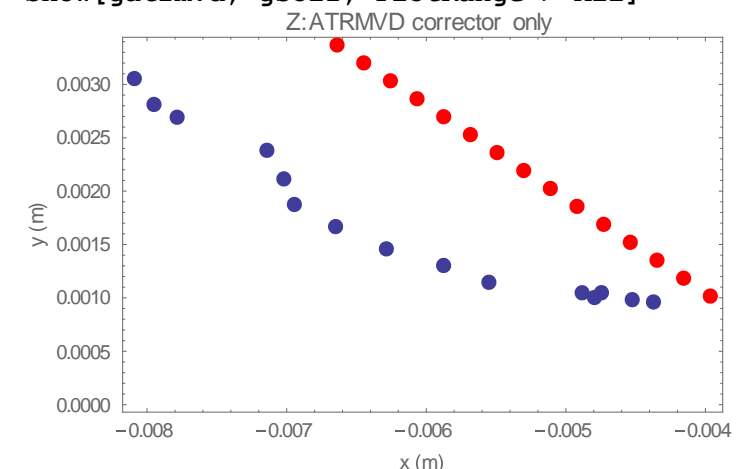

Consolidate with all the data

aerrorhv1 [Isol2_, $\left.f \_\right]:=\operatorname{Module}[\{i, j, s, x, y, x e n d\}$, $\mathbf{s}=0$;

For $[i=1, i<=$ Length $[1$ trmh $], i++$,

xend $=x v 3$ wires $[1 \operatorname{trmh}[[i, 1]], 0$, Isol2, $f, x 3 v]$;

$\mathbf{x}=$ xend $[[1,1]]+\Delta \mathbf{x}$;

$\mathrm{y}=\mathrm{xend}[[3,1]]+\Delta \mathrm{y}$;

$s+=(x-(1 \operatorname{trmh}[[i, 2]]))^{2}+(y-(1 \operatorname{trmh}[[i, 3]]))^{2}$; 
] ;
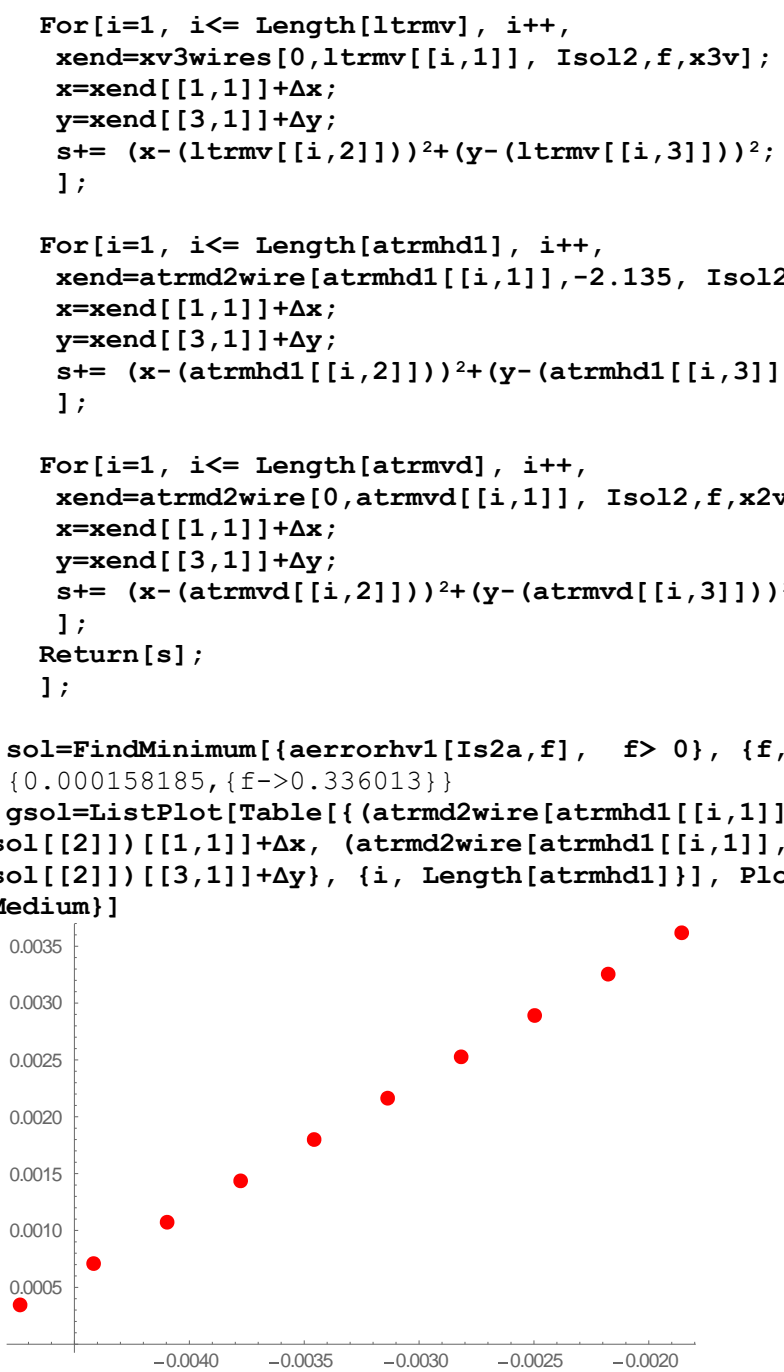

Show [gatrmhd1, gsol, PlotRange-> All]

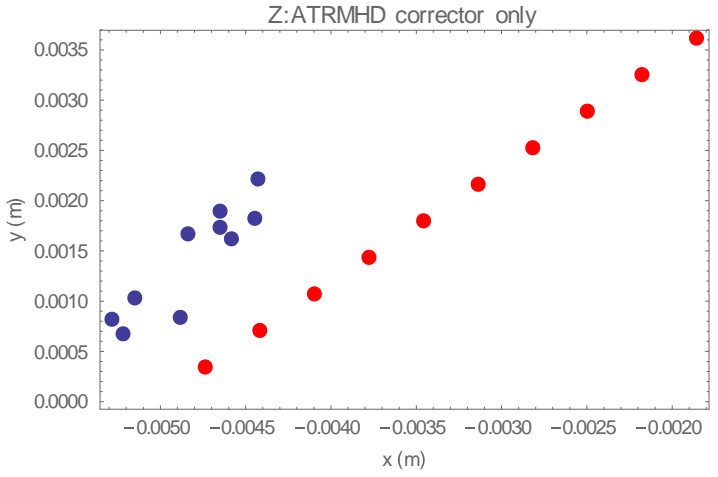

gsol1=ListPlot [Table $[\{(\operatorname{atrmd2wire}[0, \operatorname{atrmvd}[[i, 1]]$, Is $2 a, f, \mathbf{x} 2 v] /$. sol $[[2]])[[1,1]]+\Delta \mathbf{x}$ (atrmd2wire $[0, \operatorname{atrmvd}[[i, 1]], I s 2 a, f, x 2 v] /$. sol $[[2]])[[3,1]]+\Delta y\},\{i$, Length $[a t r m v d]\}]$, PlotStyle-> Red, PlotMarkers-> \{Automatic, Medium\}] 


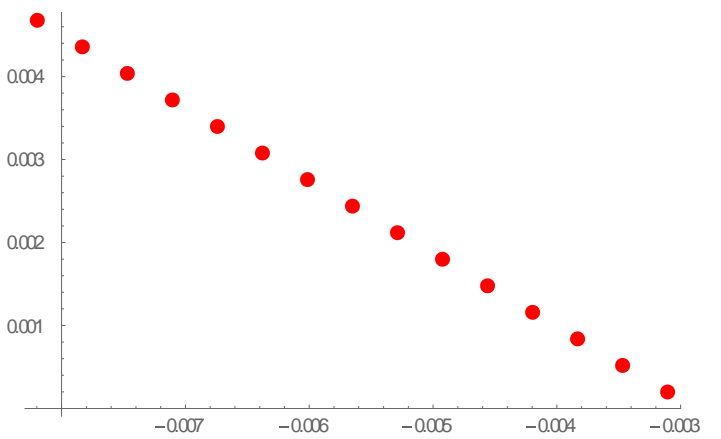

Show [gatrmvd, gsoll, PlotRange-> All]

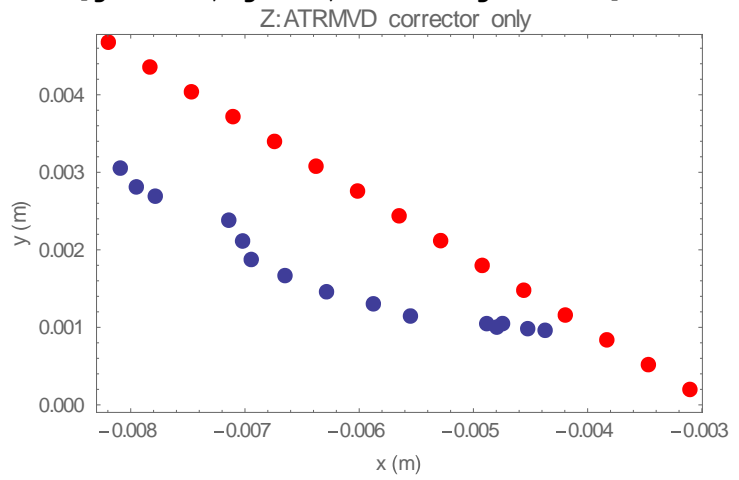

gsol=ListPlot $[$ Table $[\{(x v 3 w i r e s[1 \operatorname{trmh}[[i, 1]], 0, \operatorname{Is} 2 a, f, x 3 v] / . \operatorname{sol}[[2]])[[1,1]]+\Delta x$, $(x v 3 w i r e s[1 \operatorname{trmh}[[i, 1]], 0, \operatorname{Is} 2 a, f, x 3 v] / . \operatorname{sol}[[2]])[[3,1]]+\Delta y\},\{i$, Length $[1 \operatorname{trmh}]\}]$, PlotStyle-> Red, PlotMarkers-> \{Automatic, Medium\}]

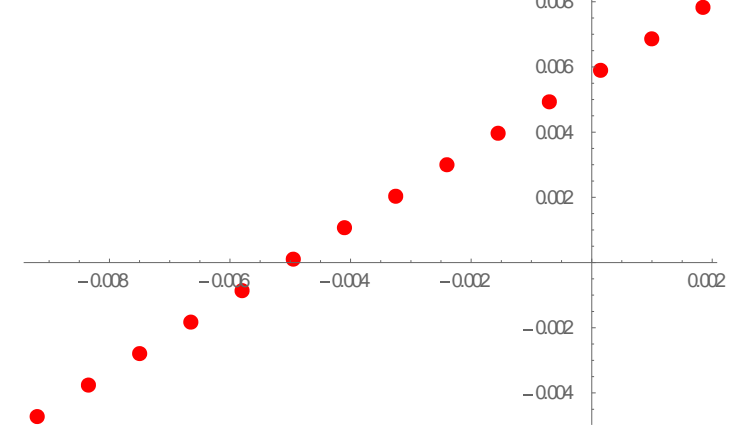

Show [gltrmh, gsol, PlotRange-> All]

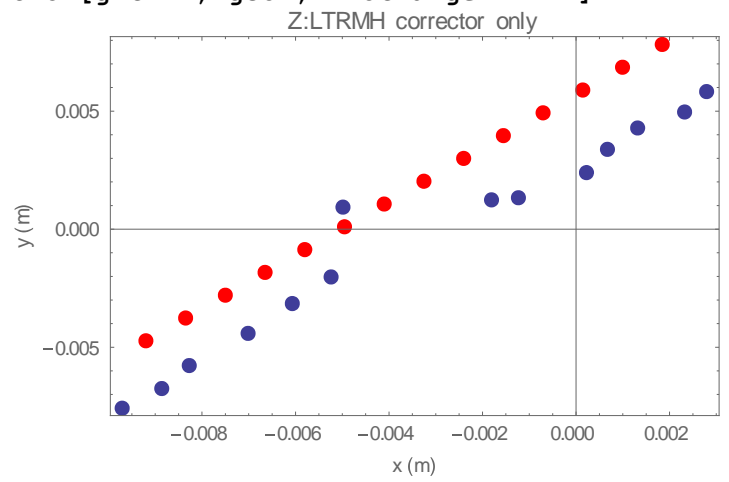

gsol1=ListPlot [Table $[\{(x v 3 w i r e s[0,1 \operatorname{trmv}[[i, 1]], \operatorname{Is} 2 a, f, x 3 v] / . \operatorname{sol}[[2]])[[1,1]]+\Delta x$, $(x v 3 w i r e s[0,1 \operatorname{trmv}[[i, 1]], I s 2 a, f, x 3 v] / . \operatorname{sol}[[2]])[[3,1]]+\Delta y\},\{i$, Length $[1 \operatorname{trmv}]\}]$, PlotStyle-> Red, PlotMarkers-> \{Automatic, Medium\}] 


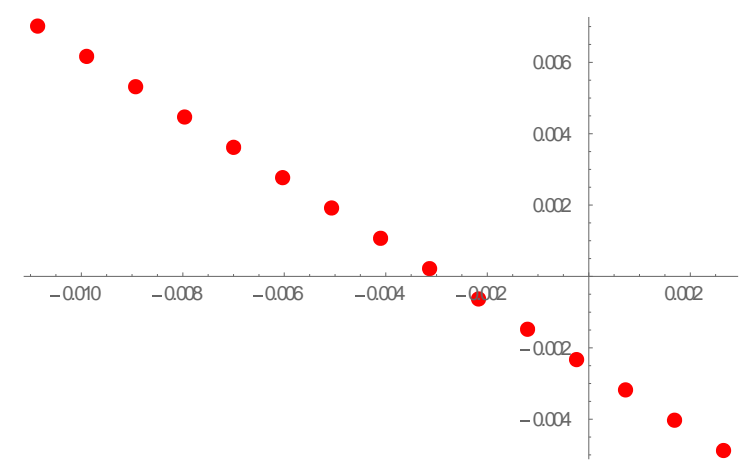

Show [gltrmv, gsol1, PlotRange-> All]

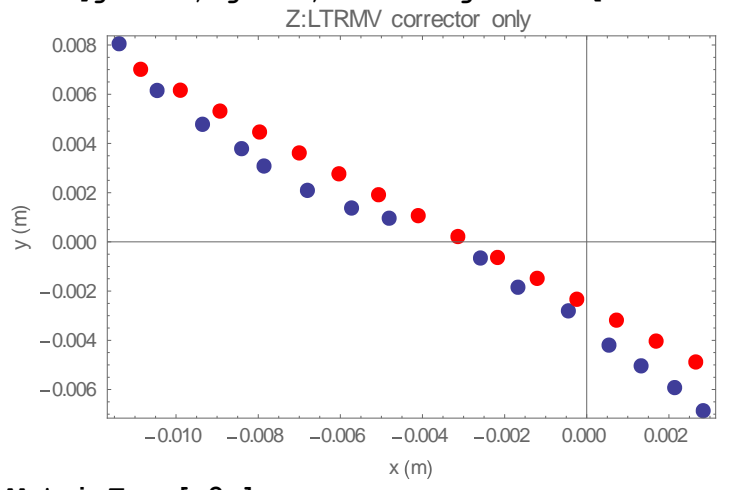

MatrixForm [x2v]

( \{

$\{0.000819577\}$,

$\{-0.00210235\}$,

$\{-0.0170988\}$,

$\{0.0300074\}$

\})

MatrixForm [x3v]

( \{

$\{-0.000181161\}$,

$\{-0.00210235\}$,

$\{-0.0150923\}$,

$\{0.00421531\}$

\})

$\mathrm{x} 0$ vector

Now, we can calculate what $x 0$ just upstream of $z: \operatorname{atrm}^{*} u$

$\mathbf{x} 0 \mathrm{v}=(\{$

$\{\mathrm{x} 0\}$,

$\{\mathrm{xp} 0\}$,

$\{\mathrm{y} 0\}$,

\{yp0\}

\});

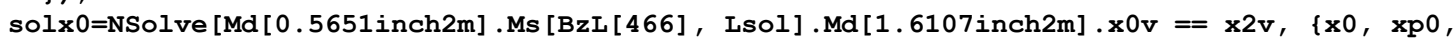
y0, yp0\}]

$\{\{\mathrm{x} 0->0.0136122, \mathrm{xp} 0->0.0257966, \mathrm{y} 0->-0.00866756, \mathrm{yp} 0->-0.0172601\}\}$

For curiosity at the source, the $z$ position of the source is about $7 \mathrm{~cm}$ from the edge of the cube,

$\mathrm{xsv}=(\mathfrak{f}$

$\{x s\}$,

$\{x p\}$,

$\{y s\}$

\{yps\}

\});

NSolve [Md[1.6107inch2m $\left.+71^{-2}\right] . x s v==(x 0 v /$. First[solx0]), \{xs, xps, ys, yps \}]

$\{\{\mathrm{xs}->0.0107511$, xps->0.0257966,ys->-0.00675322,yps->-0.0172601\}\}

Check 22 Dec 2011 Measurements

The correctors zatrm*u

From the 13 Dec 2011 measured data

zatrmhu [Ic_] $:=(1$

$\{0\}$

$\{0.172908 \pi / 180$. IC $\}$, 


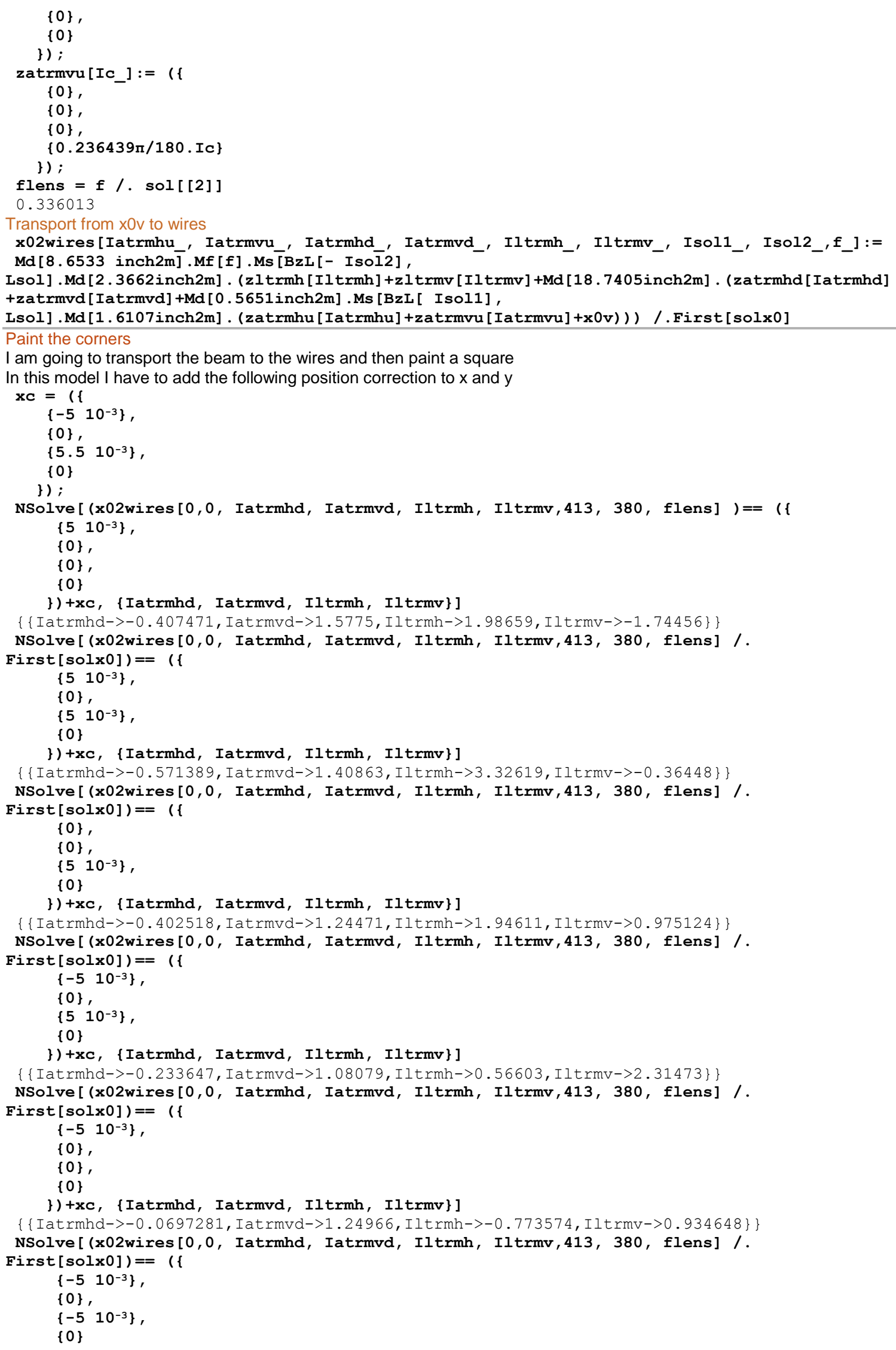


\}) $+x c$, \{ Iatrmhd, Iatrmvd, Iltrmh, Iltrmv\} ]

$\{\{$ Iatrmhd->0.0941905, Iatrmvd->1.41853, Iltrmh->-2.11318, Iltrmv->-0.445432\}\} NSolve [ (x02wires [0,0, Iatrmhd, Iatrmvd, Iltrmh, Iltrmv, 413, 380, flens] /. First $[$ solx 0$])==(\{$

$\{0\}$,

$\{0\}$,

$\left\{-510^{-3}\right\}$

$\{0\}$

\}) + xc , \{ Iatrmhd, Iatrmvd, Iltrmh, Iltrmv\} ]

$\{$ I Iatrmhd->-0.0746808, Iatrmvd->1.58245, Iltrmh->-0.733098, Iltrmv->-1.78504\}\}

NSolve [ (x02wires [0,0, Iatrmhd, Iatrmvd, Iltrmh, Iltrmv,413, 380, flens] /.

First $[$ solx 0$])==(\{$

$\left\{510^{-3}\right\}$

$\{0\}$,

$\left\{-510^{-3}\right\}$,

$\{0\}$

\}) $+x c$, I Iatrmhd, Iatrmvd, Iltrmh, Iltrmv\} ]

$\{\{$ Iatrmhd->-0.243552, Iatrmvd->1.74637, Iltrmh->0.646982, Iltrmv->-3.12464\}\}

NSolve [ (x02wires [0,0, Iatrmhd, Iatrmvd, Iltrmh, Iltrmv,413, 380, flens] /.

First $[$ solx 0$])==(\{$

$\left\{010^{-3}\right\}$,

$\{0\}$,

$\left\{010^{-3}\right\}$,

$\{0\}$

\}) $+x c$, \{ Iatrmhd, Iatrmvd, Iltrmh, Iltrmv\} ]

$\{\{$ I atrmhd->-0.238599, Iatrmvd->1.41358, Iltrmh->0.606506, Iltrmv->-0.404956\} \} 


\section{A.2 RMS Emittance Calculation}

Analysis of Emittance Probe Data

Read in the data from raw data.dat

data = ReadList ["C:/Documents and Settings/karns/My Documents/Mathematica

Simulations/06Feb2012b/06Feb2012a/Inside RF/Horizontal/H2070.dat", Table [Number, $\{i$,

23\}] ] ;

First column is emittance probe position. The 2 nd column is Z:ATOR, 3rd column is voltage on wire 0,4 th column is voltage on wire 1 etc.

I will construct an array with the following representation $\{\mathrm{x}$, wire number, $\mathrm{V}\}$.

Remove the last wire which is stuck at $9.99 \mathrm{~V}$

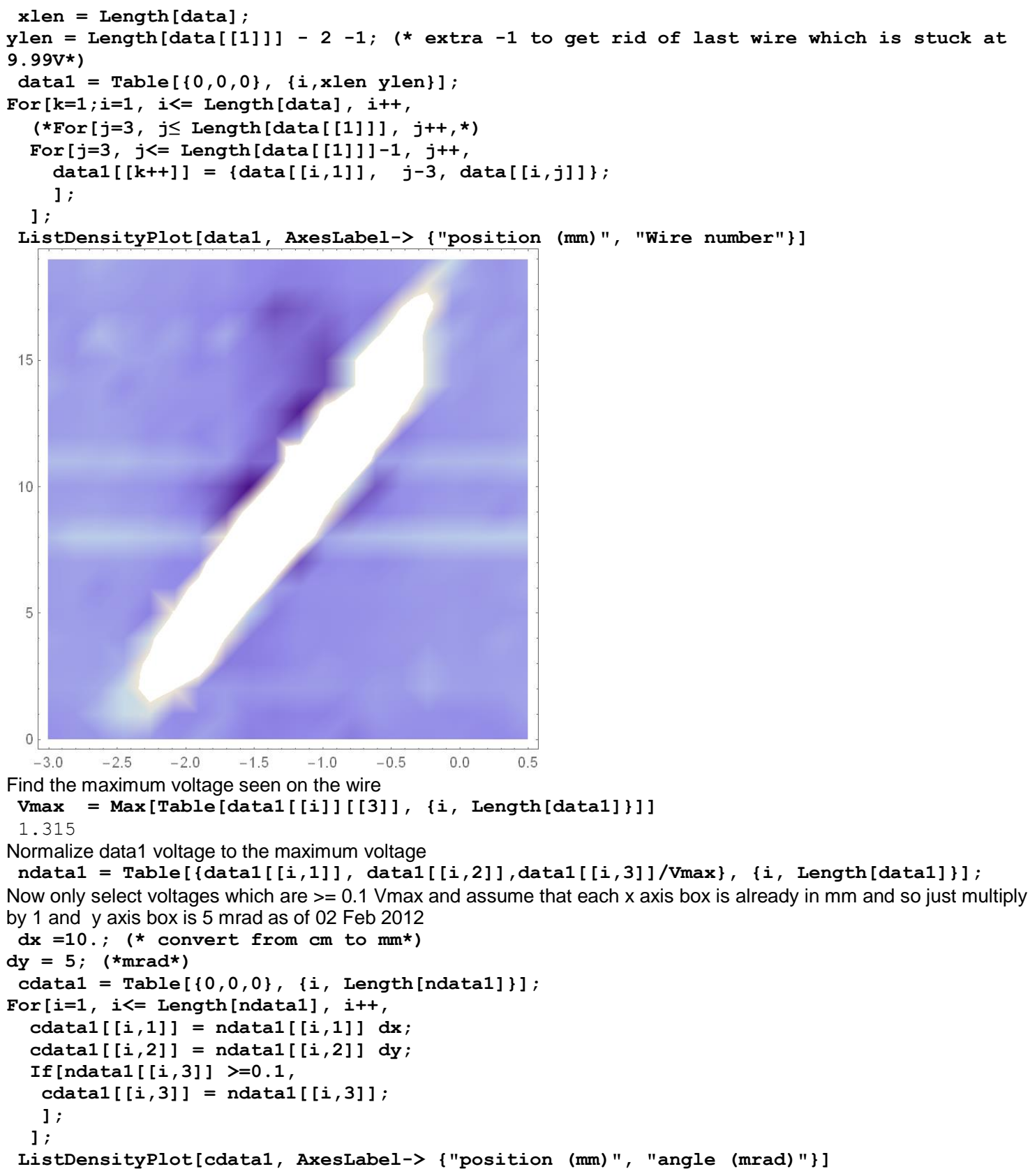




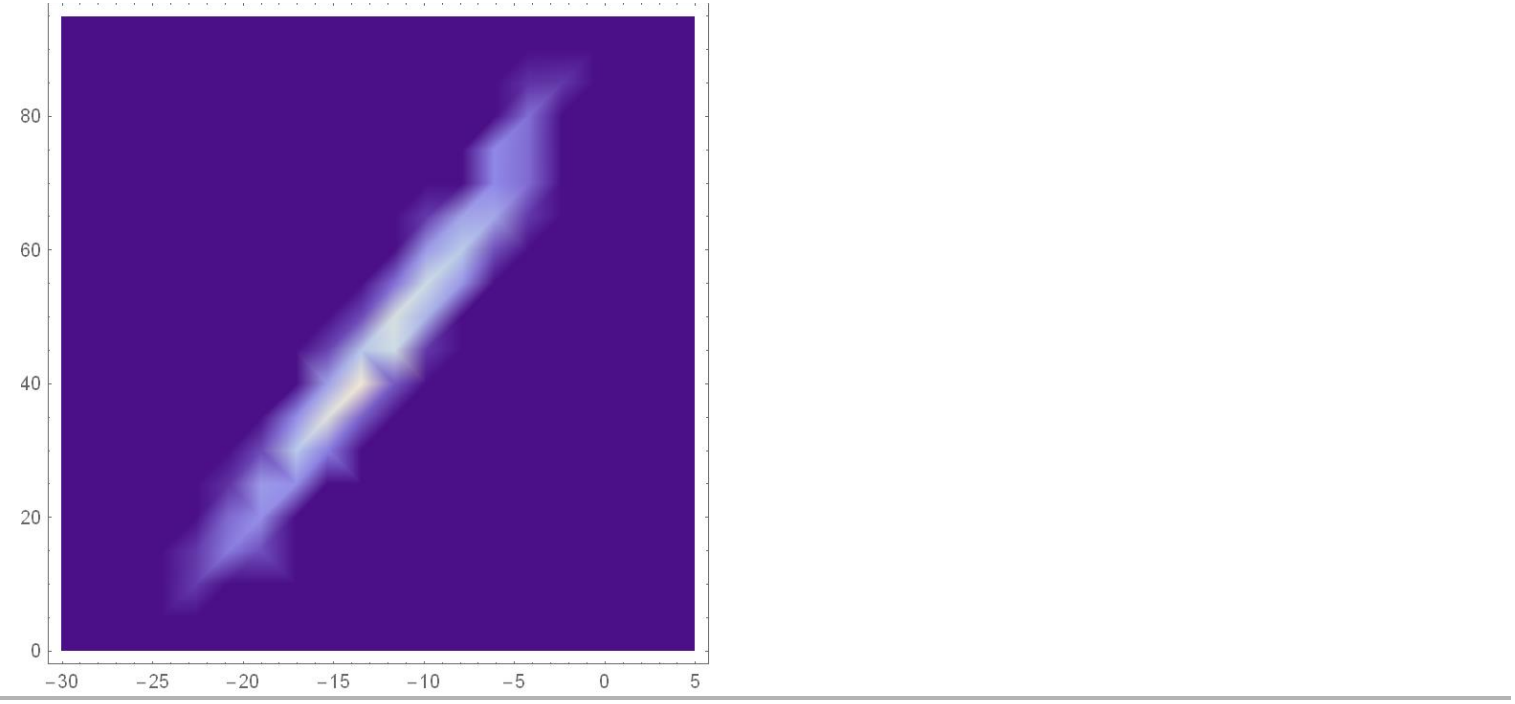

Mean values and Emittance

First I'm creating a probability distribution function $P[x, y]$. Make sure that $P[x, y] / P$ tot $=1$

Ptot $=$ Sum[cdata1 $[[i]][[3]],\{i$, Length[cdatal $]\}] d x d y$

751.288

Define the function $P[n x, n y]$

$\mathrm{P}[\mathrm{nx}$, ny_] :=cdatal [ [ $\mathrm{nx}-1)$ ylen + ny ] ] [3] ]

The mean $x$ position using

Integral[ $x P[x, y],\{x,-x \max , x \max \},\{y,-y m a x, y m a x\}] / P t o t$

First extract out $x$ and $y$ values form cdata1

xvalue $=$ Table $[$ cdatal $[[i$ ylen, 1$]],\{i, x l e n\}]$

yvalue $=$ Table $[$ cdatal $[[i, 2]],\{i$, ylen $\}]$;

meanX $=$ Sum $[$ xvalue $[[n x]] P[n x, n y],\{n x, x l e n\},\{n y, y l e n\}] d x d y /$ Ptot

$-12.294$

meanY $=\operatorname{Sum}[$ yvalue $[[n y]] P[n x, n y],\{n x, x l e n\},\{n y, y l e n\}] d x d y /$ Ptot

46.2678

xrms2 = Sum $\left[(x v a l u e[[n x]]-\operatorname{meanX})^{2} P[n x, n y],\{n x, x l e n\},\{n y, y l e n\}\right] d x d y / P t o t$

22.8075

yrms2 = Sum $\left[(y v a l u e[[n y]]-\operatorname{meanY})^{2} P[n x, n y],\{n x, x l e n\},\{n y, y l e n\}\right] d x d y / P t o t$ 290.298

xyrms $=\operatorname{Sum}[(x v a l u e[[n x]]-\operatorname{meanX})($ yvalue $[[n y]]-m e a n Y) P[n x, n y],\{n x, x l e n\},\{n y$,

ylen\} ] dx dy/Ptot

78.5184

Emittance

Using rms definition in units of $\pi \mathrm{mm}$ mrad

$\operatorname{crms}=\operatorname{Sqrt}\left[\mathrm{xrms} 2\right.$ yrms2 - xyrms $\left.^{2}\right]$

21.3501

For $750 \mathrm{keV}$ beam, $\beta y$ is 0.040032 . For $700 \mathrm{keV}$ beam $\beta y=0.0386195$

$\beta Y=0.0386195$

0.0386195

normalized rms emittance

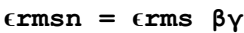

0.82453

Calculate $\alpha$ and $\beta$

Using $\sigma x=\operatorname{Sqrt}[\beta \epsilon]$, I can calculate $\beta$ from $\epsilon$ rms and $\sigma x=x r m s$

$\beta=$ xrms $2 / \mathrm{\epsilon rms}\left({ }^{*} \mathrm{~mm} / \mathrm{mrad}=\right.$ metres $\left.{ }^{*}\right)$

1.06826

From $\sigma p=\operatorname{Sqrt}[y \epsilon]$, I can calculate $y$ from $\epsilon$ rms and $\sigma p=y r m s$

$\gamma=\mathrm{yrms} 2 / \mathrm{\epsilon rms}$

13.597

Using the relationship $\beta y-\alpha^{2}=1$, I can solve for $\alpha$ and will need to pick the correct sign for the tilt of the ellipse. For the ellipse we have here, $\alpha<0$

$\alpha=-\operatorname{Sqrt}[\beta \gamma-1]$

$-3.67766$

Plots

The Twiss parameter ellipse equation

twiss $\left[\alpha_{-}, \beta_{-}, Y_{-}, \epsilon_{-}, x_{-}, x_{-}\right]:=\gamma x^{2}+2 \alpha x \times p+\beta x^{2}-\epsilon$

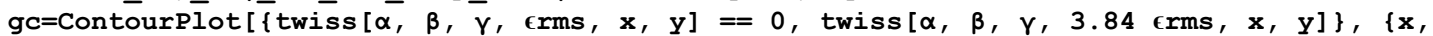

First[xvalue] - meanX, Last[xvalue] - meanX $\},\{y$, First[yvalue] - meanY, Last[yvalue] - 


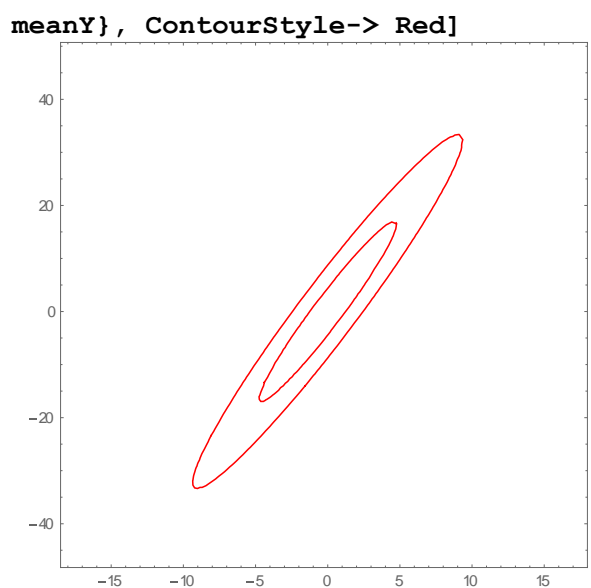

Create cdata2 so that it is centred at meanX and meanY

cdata2 = Table $[\{\operatorname{cdata} 1[i, 1]]-\operatorname{meanX}, \operatorname{cdata} 1[i, 2]]-\operatorname{meanY}, \operatorname{cdata} 1[i, 3]]\},\{i$, Length [cdata1] \}] ;

gd=ListDensityPlot [cdata2, Frame-> True, FrameLabel-> \{"x (mm) ", "x' (mrad)" \}, PlotLabel-> "Emittance", ColorFunction-> "SouthwestColors"]

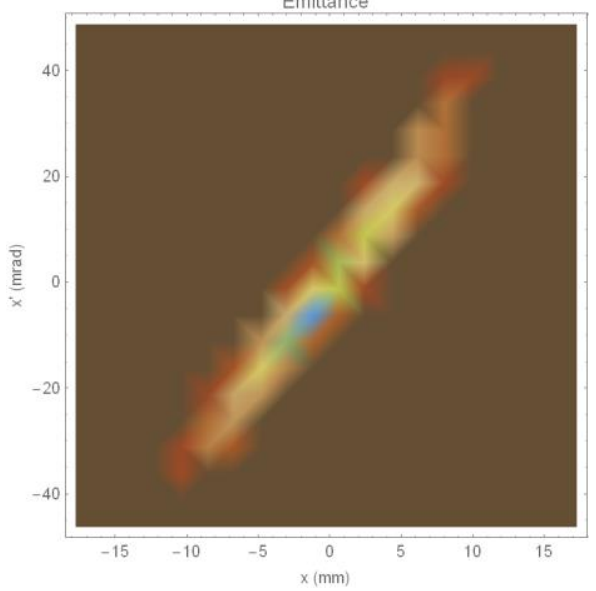

Show $[g d, g c]$

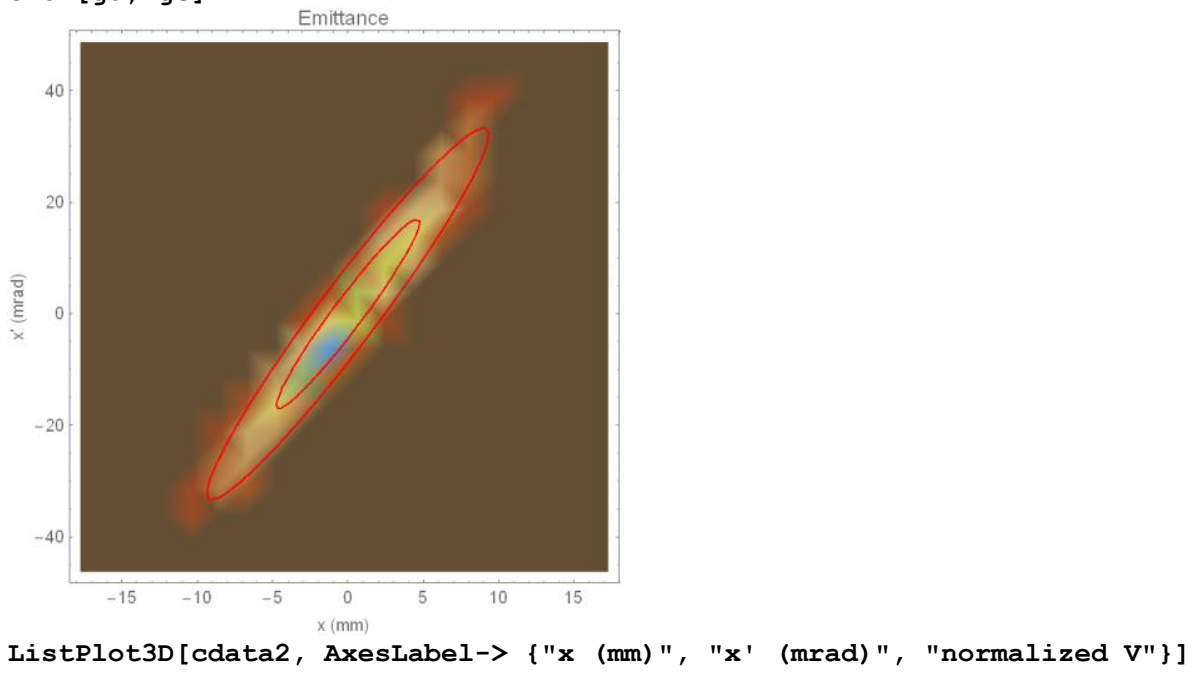




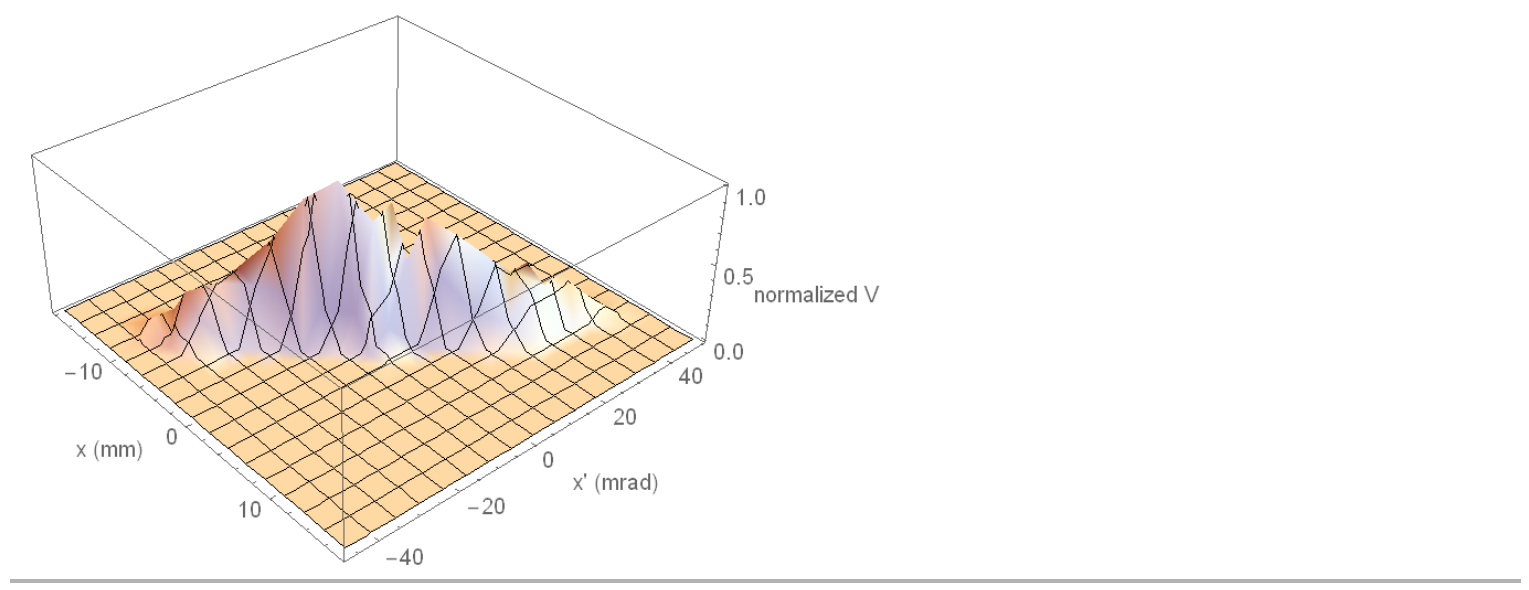

Projections

px $=$ Table $[\{$ cdata2 $[[(i-1) * y l e n+1,1]], 0\},\{i, x l e n\}]$;

For $[i=1, i<=x l e n, i++$,

$\operatorname{px}[[i, 2]]=\operatorname{Sum}[$ cdata2 $[[(i-1) y l e n+j, 3]],\{j, y l e n\}] ;$

] ;

ListPlot[px, Joined-> True, AxesLabel-> \{"x (mm)", "Integrated Voltage" $\}$ Integrated Voltage

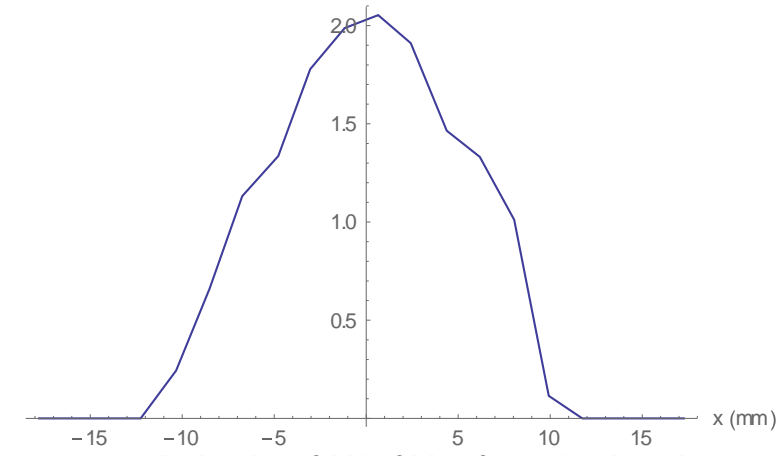

py $=$ Table $[\{$ cdata $[[i, 2]], 0\},\{i, y l e n\}]$;

For $[i=1, i<=$ ylen, $i++$,

py $[i, 2]]=\operatorname{Sum}[\operatorname{cdata} 2[j, 3]],\{j, i$, Length $[$ cdata2 $], y$ len $\}]$;

] ;

ListPlot[py, Joined-> True, AxesLabel-> \{"x' (mrad)", "Integrated Voltage" \}]

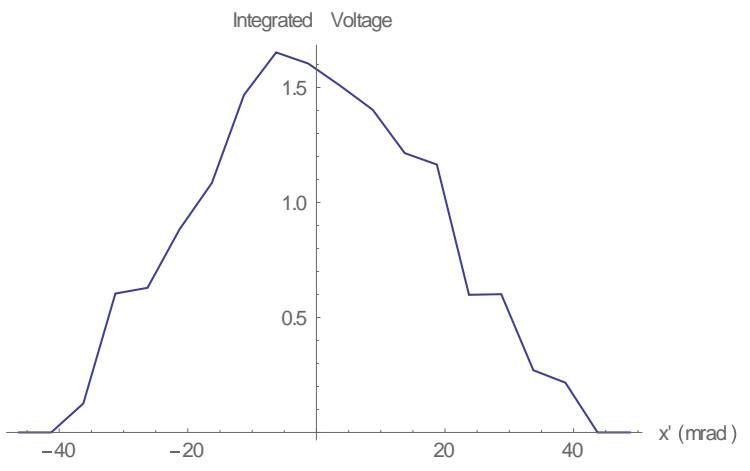




\section{A.3 Time-of-Flight Calculations (Time Domain)}

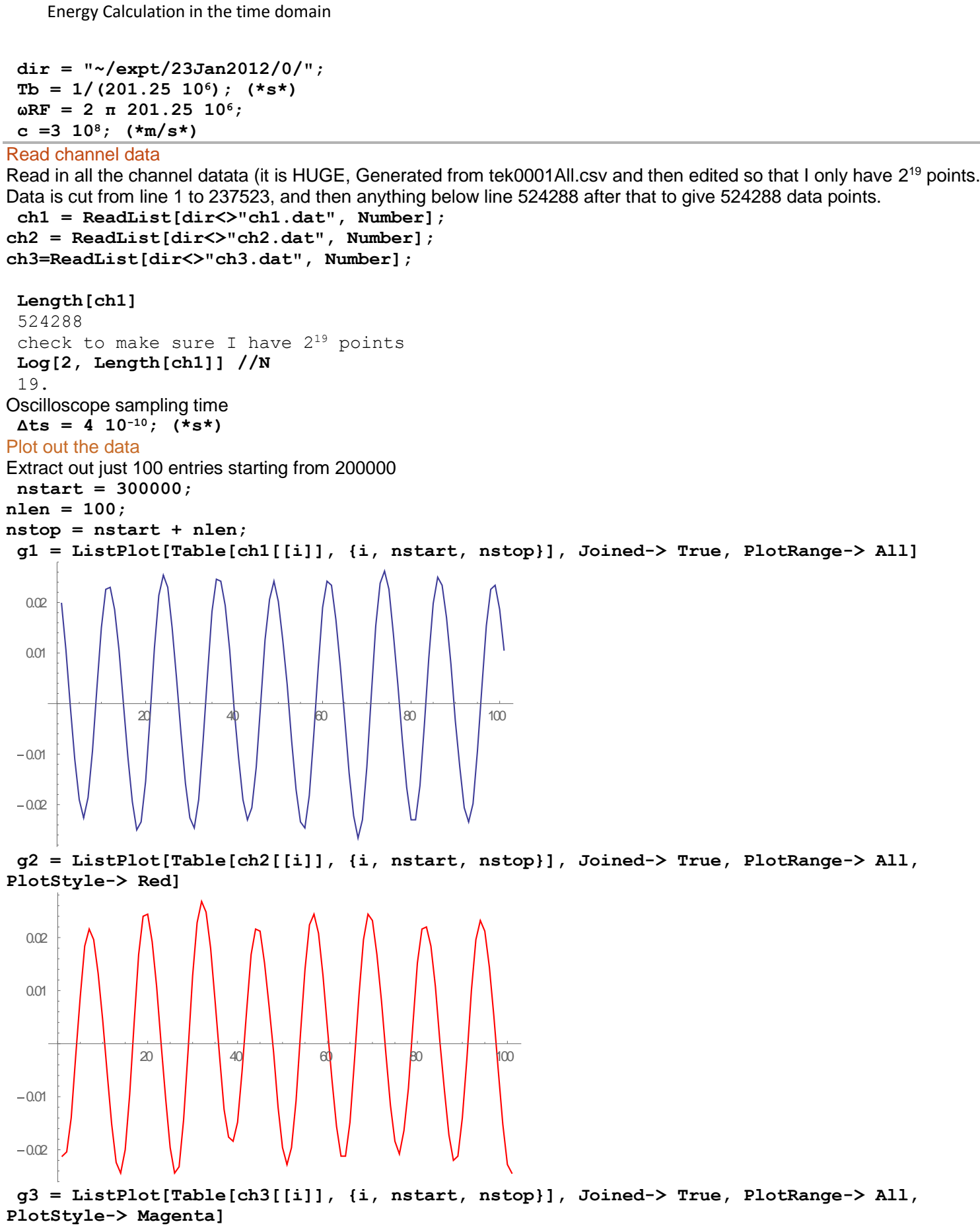

Read in all the channel datata (it is HUGE, Generated from tek0001All.csv and then edited so that I only have $2^{19}$ points. Data is cut from line 1 to 237523 , and then anything below line 524288 after that to give 524288 data points.

g3 = ListPlot $[$ Table $[$ ch3 $[i]],\{i$, nstart, nstop $\}]$, Joined-> True, PlotRange-> All, PlotStyle-> Magenta] 


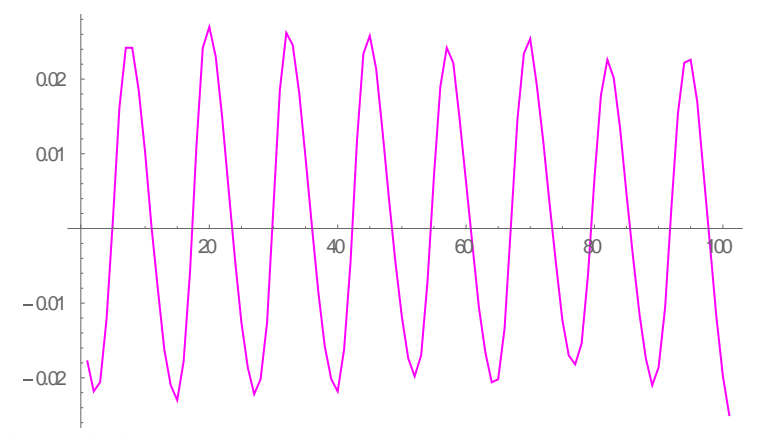

Interpolation

$f 1=$ Interpolation $[$ Table $[\{i-n s t a r t, \operatorname{ch} 1[i]]\},\{i$, nstart, nstop $\}]]$;

f2 = Interpolation[Table [\{i-nstart, $\operatorname{ch} 2[i]]\},\{i$, nstart, nstop $\}]]$;

f3 =Interpolation [Table $[\{i-n s t a r t, \operatorname{ch} 3[[i]]\},\{i$, nstart, nstop $\}]$;

Calculate energy

Using button 1 and button2

$P \operatorname{lot}[\{f 1[x], f 2[x]\},\{x, 1,25\}]$

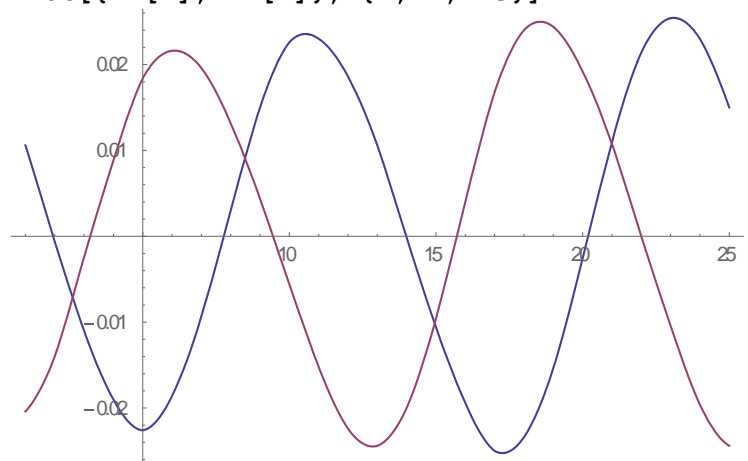

Measure the distance between peaks. Assuming that ch1 is always early, then the time between the first blue peak and the 1 st read peak AFTER the first blue peak is

$\mathrm{n} 1 \mathrm{l}$ FindRoot $[\mathrm{f} 1[\mathbf{x}],\{\mathbf{x}, 15\}]$

$\{x->13.982\}$

n2 = FindRoot $[f 2[y],\{y, 22\}]$

$\{y->22$.

$\Delta t=(y-x) \Delta t s \quad /$ Join $[\mathrm{n} 1, \mathrm{n} 2]$

$3.20718 * 10^{-9}$

$\omega R F \Delta t 180 / \pi$ (* deg*)

232.36

len $=0.153$

0.153

$\beta c=$ len $/(2 \mathrm{~Tb}+\Delta t)$

$1.16393 * 10^{7}$

$\beta=\beta c / c$

0.0387978

$\gamma=1 / \operatorname{Sqrt}\left[1-\beta^{2}\right]$

1.00075

Therefore, $\mathrm{KE}$ is

$\mathrm{mH}=93810^{6} ;\left({ }^{*} \mathrm{eV} / \mathrm{c}^{\wedge} 2 *\right)$

Solve $[\mathrm{mH}+\mathrm{ke}==\mathrm{\gamma} \mathrm{mH}$, ke]

$\{\{k e->706770\}$.

Using button 1 and button 3

$P \operatorname{lot}[\{f 1[x], f 3[x]\},\{x, 1,25\}]$ 


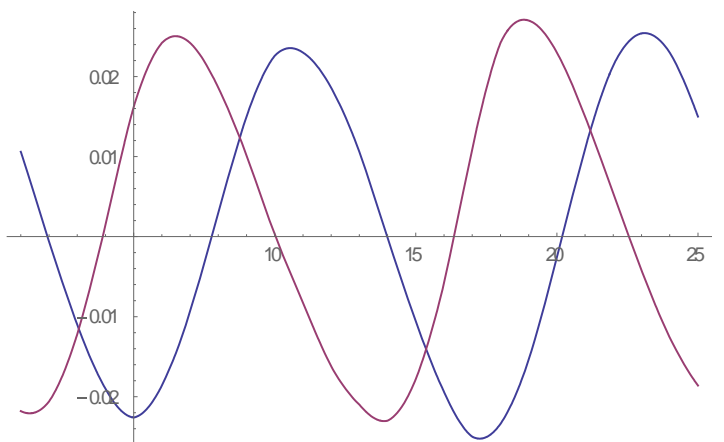

Measure the distance between peaks. Assuming that ch1 is always early, then the time between the first blue peak and the 1st read peak AFTER the first blue peak is

$\mathrm{n} 1 \mathrm{l}=\mathrm{FindRoot}[\mathrm{f} 1[\mathrm{x}],\{\mathbf{x}, \mathbf{1 5}\}]$

$\{x->13.982\}$

n2 = FindRoot $[\mathrm{f3}[\mathrm{y}],\{\mathrm{y}, 22\}]$

$\{\mathrm{y}->22.5545\}$

$\Delta t=(y-x) \Delta t s \quad /$ Join $[n 1, n 2]$

$3.42898 * 10^{-9}$

len $=0.558$

0.558

$\beta C=$ len $/(9 \mathrm{~Tb}+\Delta t)$

$1.15889 * 10^{7}$

$\beta=\beta c / c$

0.0386297

$\gamma=1 / \operatorname{sqrt}\left[1-\beta^{2}\right]$

1.00075

Therefore, KE is

$\mathrm{mH}=93810^{6} ;\left({ }^{*} \mathrm{eV} / \mathrm{c}^{\wedge} 2^{\star}\right)$

Solve $[\mathrm{mH}+k e==\vee \mathrm{mH}$, ke]

$\{\{k e->700651\}$.

Using button 2 and button 3

$P 1 \operatorname{lot}[\{f 2[x], f 3[x]\},\{x, 1,25\}]$

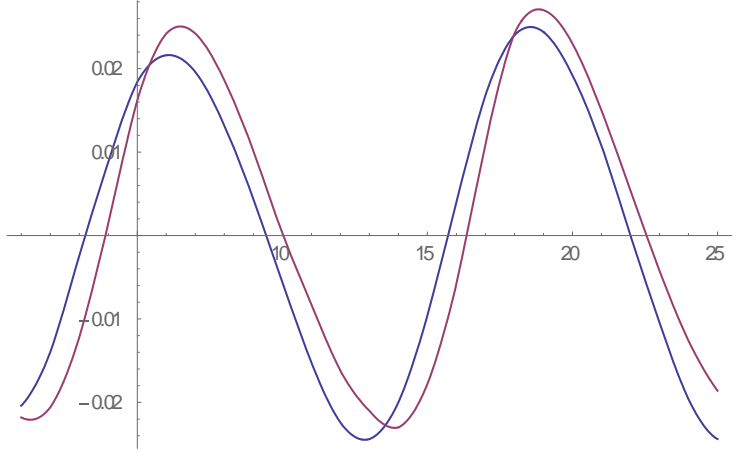

n1 = FindRoot $[f 2[x],\{x, 9\}]$

$\{x->9.4453\}$

$\mathrm{n} 2=$ FindRoot $[\mathrm{f} 3[\mathrm{y}],\{\mathrm{y}, 10\}]$

$\{\mathrm{y}->10.0223\}$

$\Delta t=(y-x) \Delta t s \quad /$ Join $[n 1, n 2]$

$2.30786 * 10^{-10}$

len $=0.405$

0.405

$\beta c=$ len $/(7 \mathrm{~Tb}+\Delta \mathrm{t})$

$1.1567 * 10$

$\beta=\beta c / c$

0.0385567

$\gamma=1 / \operatorname{Sqrt}\left[1-\beta^{2}\right]$

1.00074

Therefore, $\mathrm{KE}$ is

$\mathrm{mH}=93810^{6} ;\left({ }^{*} \mathrm{eV} / \mathrm{c}^{\wedge} 2 *\right)$

Solve $[\mathrm{mH}+k e==\vee \mathrm{mH}$, ke]

$\{\{k e->698002\}$. 


\section{A.4 Time-of-Flight Calculations (Frequency Domain)}

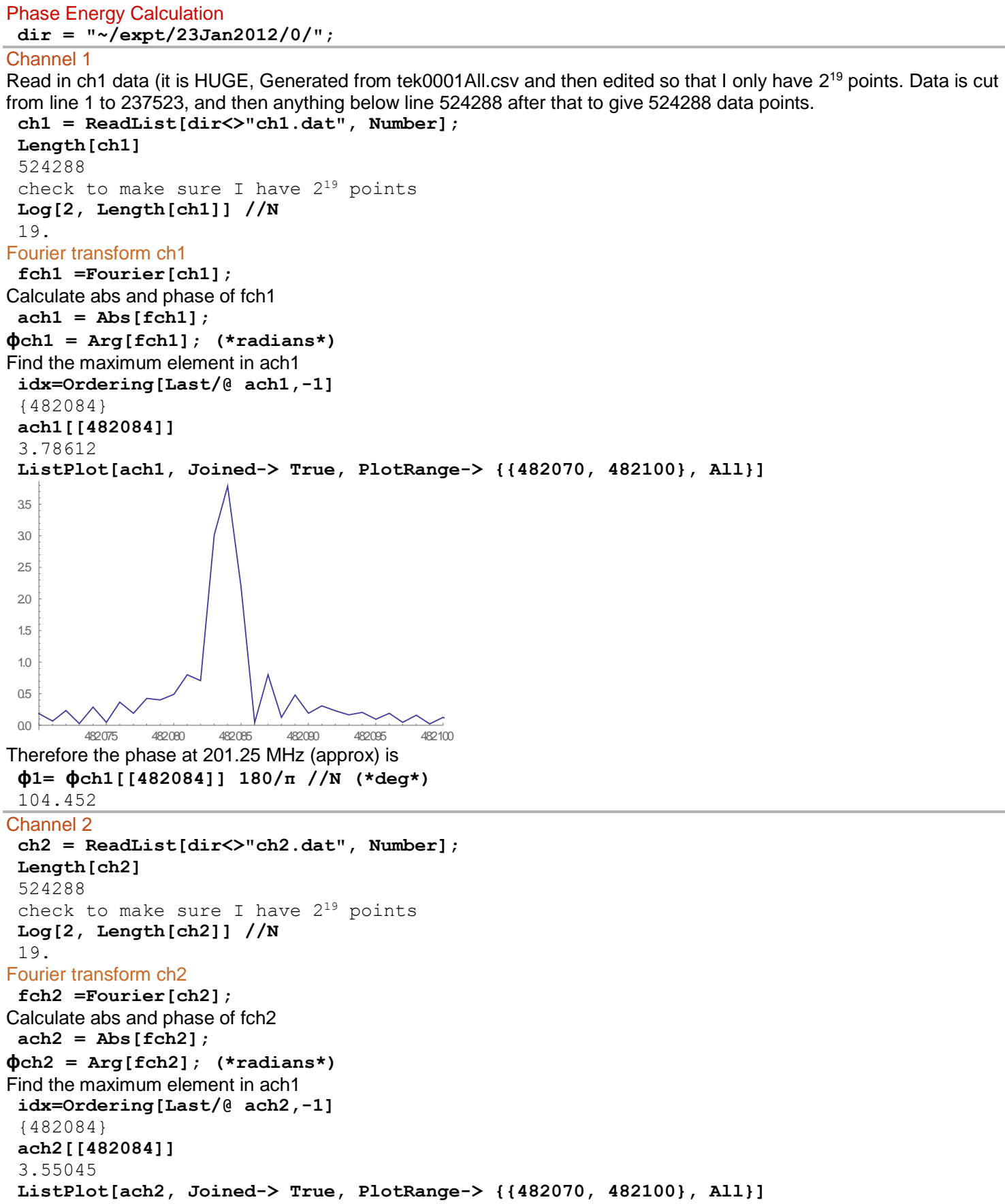




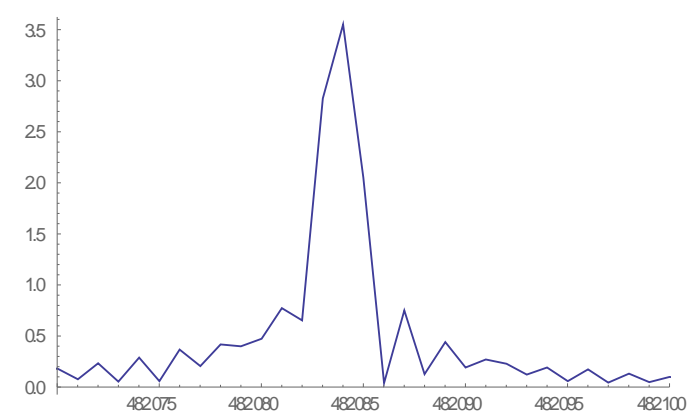

Therefore the phase at $201.25 \mathrm{MHz}$ (approx) is

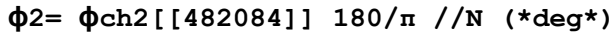

$-123.229$

Channel 3

ch3 = ReadList $[$ dir $<>" c h 3$. dat", Number $]$;

Length [ch3]

524288

check to make sure I have $2^{19}$ points

Log [2, Length [ch3]] //N

19.

Fourier transform ch3

fch3 =Fourier [ch3] ;

Calculate abs and phase of fch3

ach3 = Abs [fch3];

фch3 $=\operatorname{Arg}[f \mathrm{ch} 3] ; \quad(*$ radians $*)$

Find the maximum element in ach1

idx=Ordering [Last/ $@$ ach $3,-1]$

$\{482084\}$

ach3 [ [482084] ]

3.62245

ListPlot[ach3, Joined-> True, PlotRange-> $\{\{482070,482100\}, A l 1\}]$

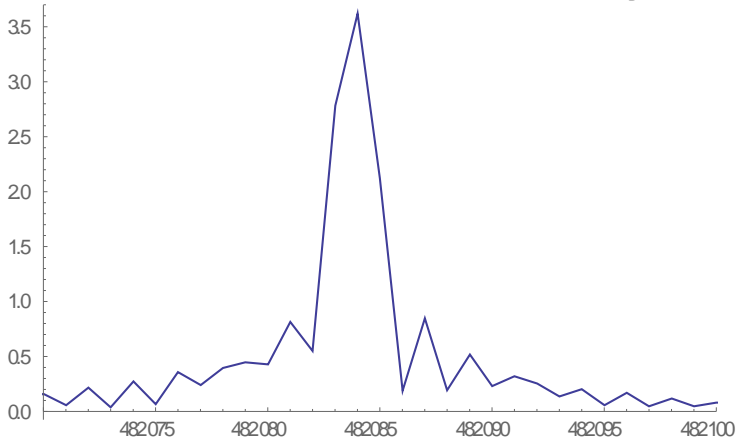

Therefore the phase at $201.25 \mathrm{MHz}$ (approx) is

$\phi 3=\phi \operatorname{ch} 3[[482084]]$ 180/n //N (*deg*)

$-137.624$

Calculate energy

Using button 1 and button2

$\Delta \phi=\phi 1-\phi 2$

227.68

$\omega R F=2 \pi 201.2510^{6}$.

$\mathrm{C}=310^{8}$;

$\Delta \phi \pi / 180$.

3.97377

total $\phi=22 \pi+\Delta \phi \pi / 180$.

16.5401

$\Delta \mathrm{t}=$ total $\phi / \omega \mathrm{RF}$

$1.30805 * 10^{-8}$

$\mathrm{v}=0.153 / \Delta t$

$1.16968 * 10^{7}$

$\boldsymbol{\beta}=\mathrm{v} / \mathrm{c}$

0.0389894

$\gamma=1 / \operatorname{Sqrt}\left[1-\beta^{2}\right]$

1.00076

Therefore, $\mathrm{KE}$ is for $\mathrm{H}$ - 


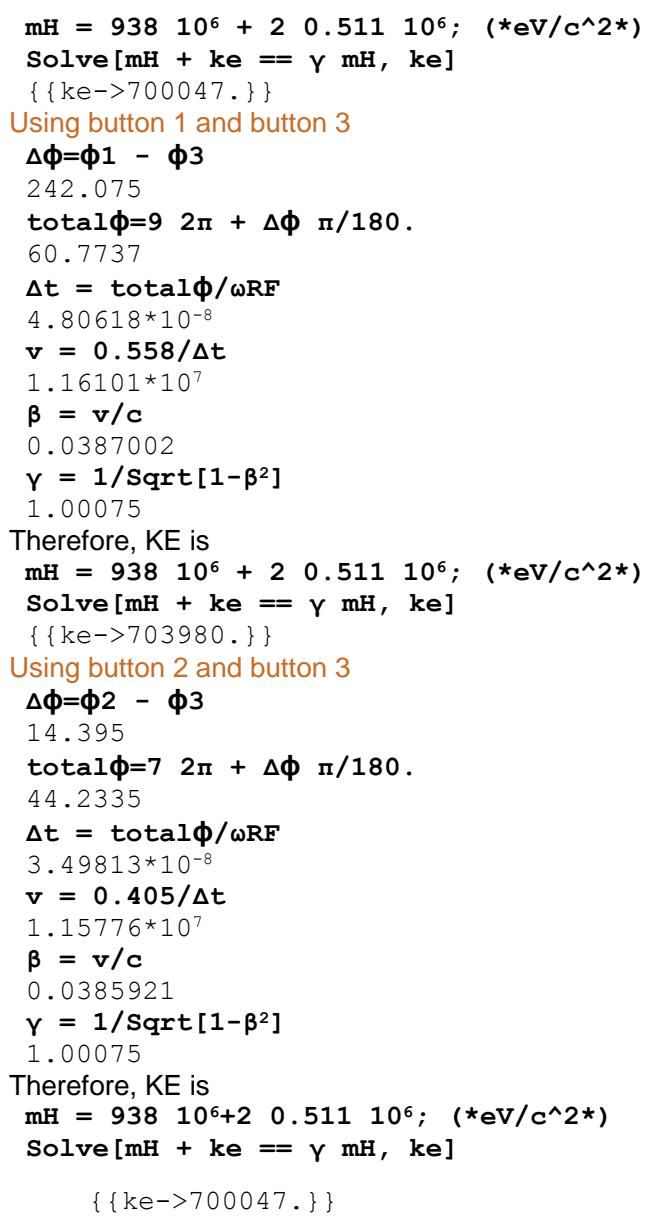


Patrick R. Karns

\begin{tabular}{|c|c|}
\hline Strengths & $\begin{array}{l}\text { - Excel in troubleshooting and problem-solving in computer and software } \\
\text { integrated systems, mechanical systems, and real world situations. } \\
\text { - Ability to learn quickly and rapidly apply new information to form new } \\
\text { solutions and methodologies. } \\
\text { - Ability to work independently and as a part of a team to achieve goals within } \\
\text { strict time constraints. } \\
\text { - Manage group members in a high stress and task-oriented environment. } \\
\text { - } \text { systems as well as C++, Fortran, Web and Java programming. }\end{array}$ \\
\hline Experience & 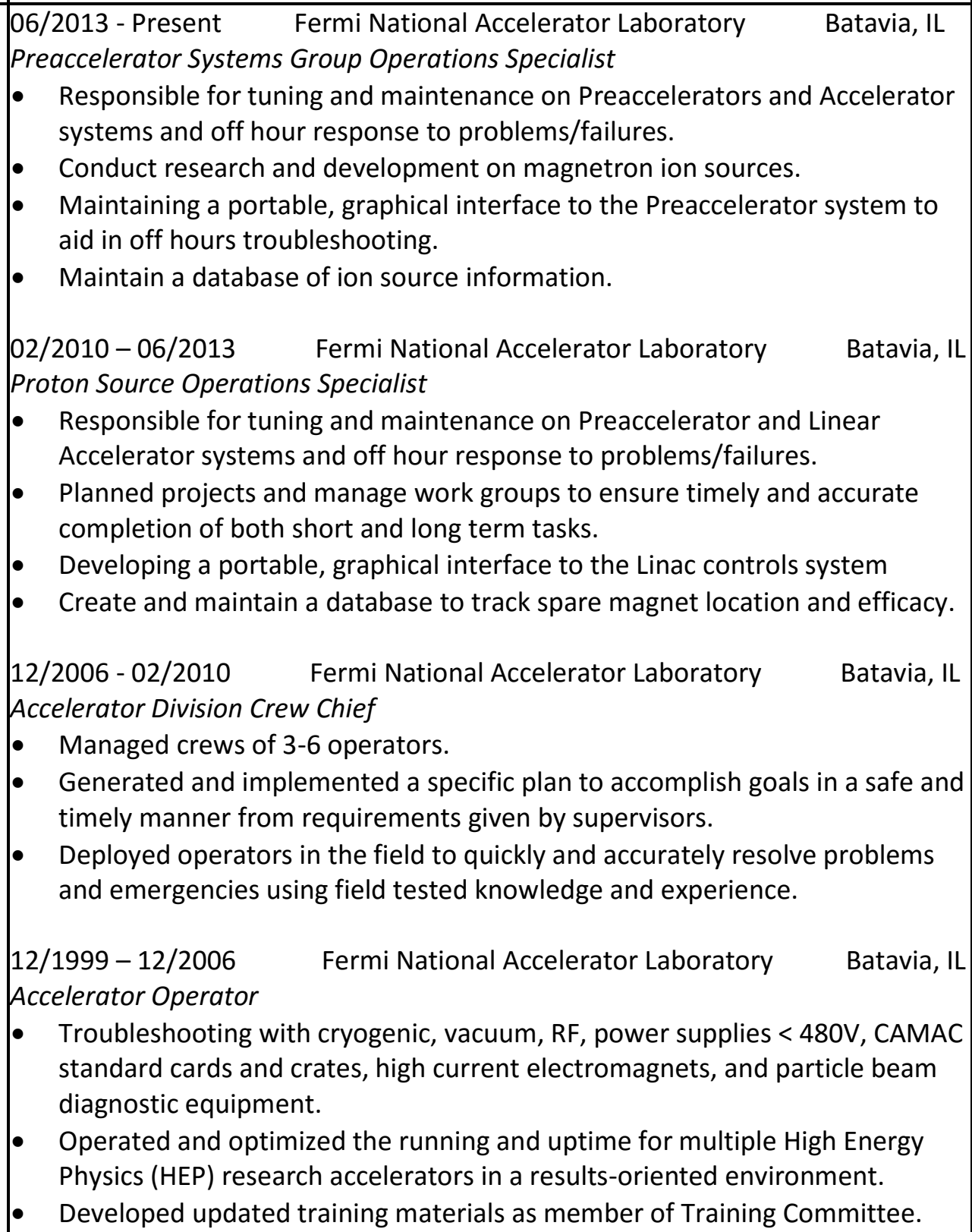 \\
\hline
\end{tabular}




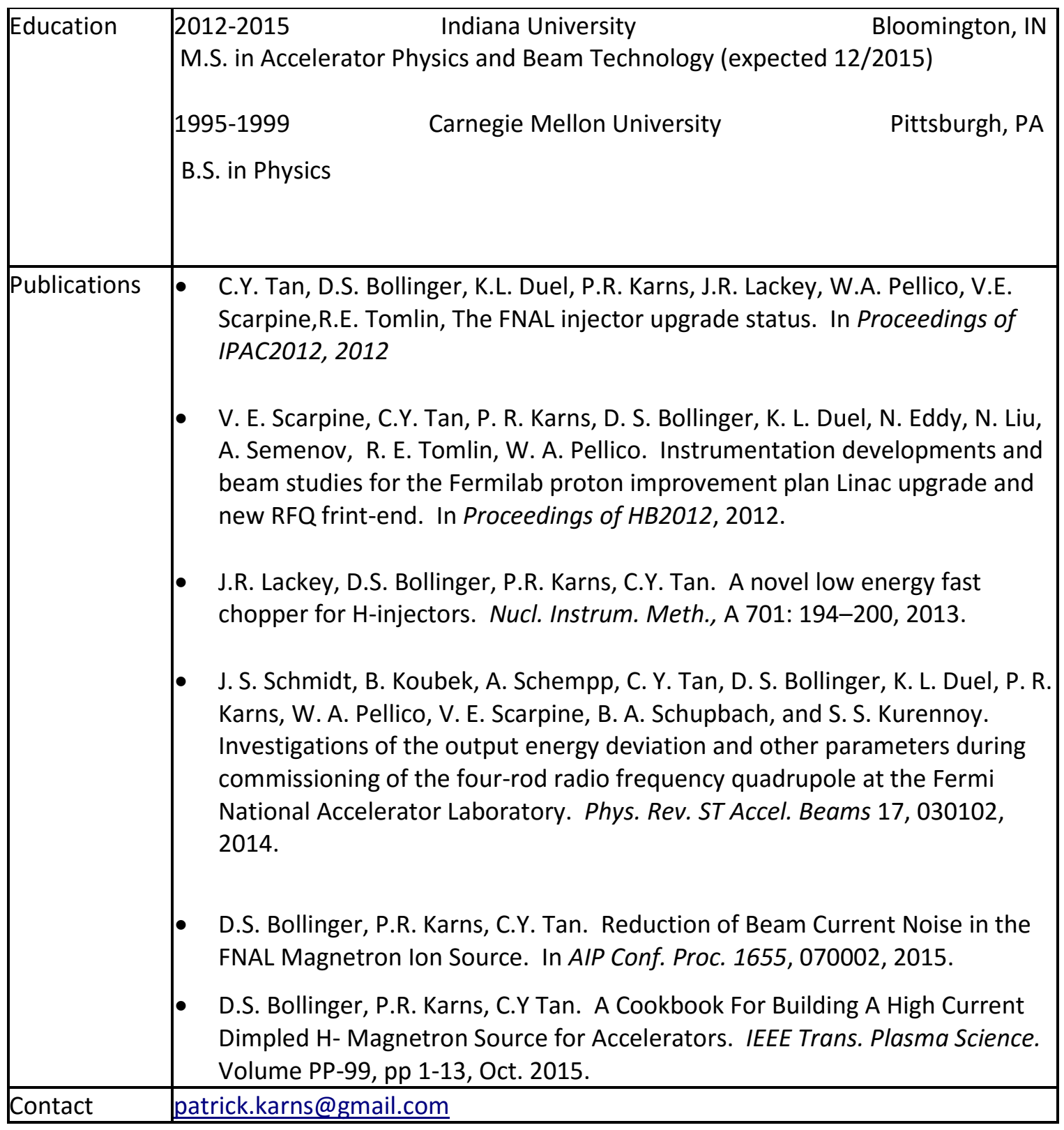

